LBL -28149

DE90 006086

\title{
Pion Correlations as a Function of Atomic Mass in Heavy Ion Collisions
}

Aaron Dean Chacon

Ph. D. Thetis

Department of Physics

University of California

and

Nuclear Science Division

Lawrence Berkeley Laboratory

1 Cyclotron Road

Berkeley, California 94720

November 26, 1989

This work was supported by the Director, Office of Energy Research, Divisjon of Nuclear Physics of the Office of High Energy and Nuclear Physics of the U.S. Depertment of Energy under Contracts DE-FG03-87ER40323 and DE-AC03-76SF00098.


DISTRIBUTION OF THIS DOCUMENT IS UNLIMITED 


\begin{abstract}
The method of two pion interferometry wh used to obtain source-size and lifetime parameters for the pions produced in heavy ion collisions. The systems ueed were $1.70 \cdot \mathrm{A} \mathrm{GeV}$ ${ }^{56} \mathrm{Fe}+\mathrm{Fe}_{1} 1.82 \cdot \mathrm{A} \mathrm{GoV}{ }^{40} \mathrm{Ar}+\mathrm{KCl}$ and $1.54 \cdot \mathrm{A} \mathrm{GeV}{ }^{05} \mathrm{Nb}+\mathrm{Nb}$, allowing for a soarch for depeadences on the atomic number. Two acceptances (centered, in the lab., at $\sim 0^{\circ}$ and $\left.4^{\circ}\right)$ were used for esch oystem, allowing a search for dependences on the viewing ancle.

The correlation functions were calculated by comparing the date semplet to background (or reference) samples madr: using the method of event mixing, where pions from different events are combined to produce a data sample in which the Bose-Einatein correIation effect is absent. The effect of the correlation function on the background samples in calculated, and a method for weighting the events to remove the residual correlation effect is presented. The effect of the apectrometer deaign on the measured correlation functions is discussed, as are methods for correcting for these effects during the data analysis.

The form of the correlation function fit allowed the source to have difierent radius pa. rameters in the direction perpendicular to the beam and parallel to the beam (although the source was asumed to be cylindrically symmetric about the beam axis). The cource parameters show an oblate cource (e.g., $R_{\perp}>R_{\|}$) for the lighter systems and an approximately spherical source for the heaviest system. When the two acceptances are compared system by system, $R_{\perp}$ for the $0^{\circ}$ acceptance afrees with $R_{\perp}$ for the $45^{\circ}$ acceptance, whereas $R_{\|}$is smaller in the $45^{\circ}$ arceptance than in the $0^{\circ}$ acceptance. The dependence on atomic number shows that $R_{\perp}$ is esentially constant and $R_{\|}$shows a large dependence on atomic number. Fits where $R_{\perp}$ was forced to equal $R_{\|}$were made for all syatems in the $4^{\circ}$ acceptance and the $\mathrm{Ar}+\mathrm{KCl}$ data are compared to earlier measurements made on the ame system with a similar acceptance. Cuts imposed on the pions' momenta show no evidence for source size dependence on pion momentum.
\end{abstract}




\section{Contents}

1 Introduction 1



1.2 Notation . . . . . . . . . . . . . . . . . . 4

2 Eistorical Roview s

2.1 Photon-Photon Correlations $\ldots \ldots \ldots \ldots \ldots \ldots \ldots$

2.2 Particle-Particle Correlation . . . . . . . . . . . . . . 6

2.3 Particle Correlations in Nuclear Collisions . . . . . . . . . . 8

3 Motivation and Thwory 11

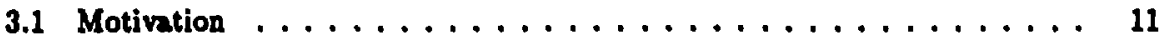

3.2 Derivation of Bowe-Einstein Correlations . . . . . . . . . . . 12

3.3 Correlation Function Used in the Fit . . . . . . . . . . . . 14

3.4 Experimental Correlation Function $\ldots \ldots \ldots \ldots \ldots \ldots$

3.5 Coherence and the $\lambda$ Parameter . . . . . . . . . . . . . 17

4 Apparatur 20

4.1 The Bevalac Accelerator . . . . . . . . . . . . . . . . 20

4.2 The Janus Spectrometer $\ldots \ldots \ldots \ldots \ldots \ldots$

4.3 Fant Electronica and Computer Readout . . . . . . . . . . . 28

s Track Inding and Particle Identification 32

5.1 Outline of the Problem . . . . . . . . . . . . . . . . 32

5.2 Hit Finding $\ldots \ldots \ldots \ldots \ldots \ldots \ldots \ldots \ldots \ldots \ldots \ldots$

5.3 Effective Edge Track Finding $\ldots \ldots \ldots \ldots \ldots \ldots \ldots$ 
5.4 Two Track Efficiency . . . . . . . . . . . . . . . . 48

5.5 Particle Identification $\ldots \ldots \ldots \ldots \ldots \ldots \ldots$

5.6 Momentum and Other Track Parameter Fitting . . . . . . . . 50

- Background Generation and Function Attins 83

6.1 Background Event Generation . . . . . . . . . . . . . . 53

6.2 Derivation of Event Mixing $\ldots \ldots \ldots \ldots \ldots \ldots \ldots$

6.3 Reidual Correlations. . . . . . . . . . . . . . . . . . . 59

6.4 Iteration Scheme for the Event Weights . . . . . . . . . . . . 60

6.5 Momentum Difference (Q) Histograms . . . . . . . . . . . . . . . 64

6.6 Background Fluctuations $\ldots \ldots \ldots \ldots \ldots \ldots \ldots \ldots$

6.7 Other Corrections $\ldots \ldots \ldots \ldots \ldots \ldots \ldots \ldots \ldots \ldots \ldots \ldots$

6.8 Fitting the Correlation Function $\ldots \ldots \ldots \ldots \ldots \ldots$

6.9 Summary of the Fitting Procedure $\ldots \ldots \ldots \ldots \ldots$. . . . . 70

7 Results Irom the Correlation Function Fits - 71

7.1 Presentation of the Data . . . . . . . . . . . . . . . . . 71

7.2 Fits to Monte Carlo Datz . . . . . . . . . . . . . . . . 72

7.3 Projections for the Data . . . . . . . . . . . . . 80

7.4 Confidence Contours for the Data . . . . . . . . . . . . . 87

7.5 Study of the Coulomb Corrections . . . . . . . . . . . . 87

7.6 Systematic Uncertainty $\ldots \ldots \ldots \ldots \ldots \ldots$

7.7 The Parameters . . . . . . . . . . . . . . . . . 96

7.8 Discustion . . . . . . . . . . . . . . . . . 102

8 Conclusions and Future Work 107

8.1 Conclusions . . . . . . . . . . . . . . . . 107

8.2 Future Work $\ldots \ldots \ldots \ldots \ldots \ldots \ldots \ldots \ldots \ldots \ldots \ldots \ldots$

A The Monte Carlo Program 115

$\begin{array}{ll}\text { Bibliography } & 117\end{array}$ 


\section{List of Figures}

4.1 The Janue apectromotor In the plan viow (1982). . . . . . . . . . . 21

4.2 The Janus apectrometer in the plan view (1986). . . . . . . . . . . 22

4.3 Contour plot of the acceptance for the Fe setups. . . . . . . . . . 27

4.4 Trizser logic part 1. . . . . . . . . . . . . . . . . . . 29

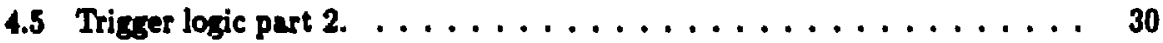

4.6 Slow Logic. $\ldots \ldots \ldots \ldots \ldots \ldots \ldots \ldots \ldots \ldots \ldots \ldots \ldots \ldots$

5.1 A triangle in a wire chamber. . . . . . . . . . . . . 34

5.2 Three hits in a wire chamber showing poesible shared wires. . . . . . 37

5.3 The Janus magnet with the set up for the $\Delta r$ calculation. . . . . . . 39

$5.4 \Delta r$ with two tracks per event. . . . . . . . . . . . . . 44

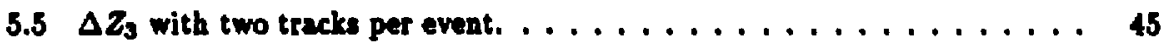

$5.6 \Delta \boldsymbol{Z}_{4}$ with two tracks per event. . . . . . . . . . . . 46

5.7 Track findiors as a function of the hit separation. . . . . . . . 47

6.1 Estimated fractional uncertainty in the background. . . . . . . . 67

$7.1\left(C\left(g_{0}\right)\right)$ for the Monte Carlo data $\ldots \ldots \ldots \ldots \ldots \ldots \ldots$

$7.2\left\langle C\left(q_{1}\right)\right)$ for the Monte Carlo datz. . . . . . . . . . . 75

$7.3\left\{C\left(q_{1}\right)\right\rangle$ for the Monte Carlo dats. . . . . . . . . . . 76

7.4 The 10 confidence contour for tho Monte Carlo data $-R_{\|}$ve. $R_{\perp}$ plane. $\quad 77$

7.5 The 10 confidence contour for the Monte Carlo data $-\boldsymbol{R}_{\perp}$ vs. $\tau$ plane. .78

7.6 The 10 confidence contour for the Monte Carlo data $-R_{\|}$vs. $\tau$ plane. . 79

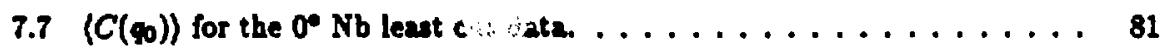

$7.8\left\langle C\left(q_{1}\right)\right\rangle$ for the $0^{\circ} \mathrm{Nb}$ least cut data. . . . . . . . . . 82 
$7.9\left\langle C\left(q_{\|}\right)\right\rangle$for the $0^{\circ} \mathrm{Nb}$ least cut data. . . . . . . . . . . 83

$7.10\left\langle C\left(q_{0}\right)\right\rangle$ for the $45^{\circ} \mathrm{Nb}$ uncut data $\ldots \ldots \ldots \ldots \ldots$

$7.11\left\langle C\left(q_{\perp}\right)\right\rangle$ for the $45^{\circ} \mathrm{Nb}$ uncut data. . . . . . . . . . . 85

$7.12\langle C($ q $)\rangle$ for the $45^{\circ} \mathrm{Nb}$ uncut data. . . . . . . . . . . . 86

7.13 The 10 confidence contour for the $0^{\circ}$ Nb cut date $-R_{\|}$va. $R_{\perp}$ plane. . 88

7.14 The $1 \sigma$ confidence contour for the $0^{\circ} \mathrm{Nb}$ cut data $-R_{\perp}$ vs. $\tau$ plane. . . 89

7.15 The $1 \sigma$ confidence contour for the $0^{\circ} \mathrm{Nb}$ cut data $-R_{\|}$vs. $\tau$ plane. . . 90

7.16 The lo confideace contour for the $45^{\circ} \mathrm{Nb}$ cut data $-R_{\|}$vs. $R_{\perp}$ plane. . 91

7.17 The $1 \sigma$ confldence contour for the $45^{\circ} \mathrm{Nb}$ cut data $-R_{\perp}$ ve. $r$ plane. . 92

7.18 The $1 \sigma$ confidence contour for the $4^{\circ} \mathrm{Nb}$ cut data $-R_{\|}$ve. $r$ plane. . 93

7.19 The $1 \sigma$ confidence contour for the $0^{*}$ Ar least cut data $-R_{\|}$vs. $T$ plane. 94

7.20 Parametern a a function of $A$ for the $45^{\circ}$ data with $R_{\perp} \equiv R_{\|} \ldots \ldots \ldots 9$

7.21 Parametern a a function of $A$ for the $45^{\circ}$ uncut data. . . . . . . 100

7.22 Parametess as a function of $A$ for the $45^{\circ}$ cut data. . . . . . . . . . 101

7.23 Parameters as a function of $A$ for the $0^{\circ}$ least cut data . . . . . 103

7.24 Parameters a a function of $A$ for the $0^{\circ}$ cut data. . . . . . . . . . 104

8.1 Pion source parametere a a function of $A^{1 / 3} \ldots \ldots \ldots$. . . . . . . 108 


\section{List of Tables}

4.1 Boam parsmeters for the runs. $\ldots \ldots \ldots \ldots \ldots \ldots \ldots \ldots \ldots 20$

4.2 Spectrometer parameters for the net-ups used. . . . . . . . . 23

4.3 Strinleas steel target composition. $\ldots \ldots \ldots \ldots \ldots \ldots \ldots \ldots . \ldots \ldots$

4.4 Spectrometer characteristics for the Fe setups. . . . . . . . . . 26

4.5 Locations of the principle components of the Janus spectrometer as ured



3.1 Effective edge parameters for one Monte Carlo track per event. . . . . . 43

5.2 Resolution for Monte Carlo simulated events. . . . . . . . . . 51

7.1 Parameters extracted from Monte Carlo simulated events. . . . . . . 73

7.2 Purameters a a function of the corrections applied, for a subert of the $0^{\circ}$ Fe data. . . . . . . . . . . . . . . . 95

7.3 Statistical "acertunties followed by estimated syntematic uncertsinties for

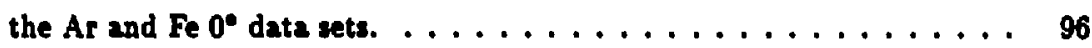

7.4 Parameters as a function of the projectile, for the $45^{\circ}$ data. . . . . . 98

7.5 Parameters as a function of the projectile, for the $45^{\circ}$ uncut data. . . . 99

7.6 Parameters as a function of the projectile, for the $45^{\circ}$ cut data. . . . . 99

7.7 Parameters as a function of the projectile, for the $0^{\circ}$ least cut data. . . . 102

7.8 Parameters as a function of the projoctile, for the $0^{\circ}$ cut data. . . . . . 105

8.1 Pion source parametern for different projectile target combinations. . . . 109

8.2 Comparison between experimental and CASCADE pion source parametera for the $45^{\circ}$ data. . . . . . . . . . . . . . . 110 
8.3 Comparison between experimental and CASCADE pion source parameters for the Fe dats . . . . . . . . . . . . . . . . . . 112 
Dedicated to the memory of Louin Grace Chacon (nco Sevier, 1933-1980), and

to all the other members of my femily - each has helped in his (or her) owa way. 


\section{Acknowledgments}

Becanes of the lerge number of people levolved, it is diffeult to recall all the people who have heiped with the experiwents which ase the main part of this thenis. Of course, all the members of the Crowe/Rumuanen group at LBL have helped at one time or another, and I would like to thank all of the members.

I thank the following people for contributiona above and beyond the call of duty:

Bill Zajc for, during the brief period when we wers both members of the group, giving me many incights into the nature of problem eolving in the real world.

Jjm Biatirlich for keeping the wire chambers and their elactronica going in apite of the fog blowing into the cave and the electronics shack.

Roy Bouingham for helping to ketp the wire chembers going, helping to increase the event read-out speed, and keeping me honeat during the analyais.

Joe Love for debugging the MBD-11 program with an ocilloncope, the only time in my life that I have sech software debugzed uaing hardware.

Tom Kozlowakj for debugzing the MBD-11 code over the telephone.

Ivanna Juxizic for relevant converations on many topics related to the analyois of particle-particle correlations.

Ken Crowe for taking me off the street, allowing me to find my own style of data analyais, and allowing me to learn from my mistakes. Of course, any errors that remain in this theis are the responsibility of the author.

Finally, I would like to thank the taxpayers of the United Staten who have chowen, through the Director, Office of Energy Rewearch, Division of Nuclear Physics of the Office of High Energy and Nuclear Physics of the U. S. Department of Energy, under contracts DE-AC03-76F00098 and DE-FG0387ER40323, to finance this work. 


\section{Chapter 1}

\section{Introduction}

This thesil is a meaurement of the size of the pion emitting region in a heavy ion collision. The measurement was made uring intensity interfesometry, which is based on the interference of identical particles due to the Bone-Einstein oymmetrization of the wave function. The large data sample sizes in this experiment allowed dropping the asumption that the size of the source measured parallel to the beam is equal to the size of the source measured perpendicular to the beani. The systems used were $1.82 \cdot \mathrm{A} \mathrm{GeV}{ }^{40} \mathrm{Ar}+\mathrm{KCl}$, 1.70 . A GeV ${ }^{36} \mathrm{Fe}+\mathrm{Fe}$ and $1.54 \cdot \mathrm{A} \mathrm{GeV}{ }^{95} \mathrm{Nb}+\mathrm{Nb},{ }^{1}$ allowing comparison of the data with existing $\mathrm{Ar}+\mathrm{KCl}$ data[1], and a search for effects depending on the size of the nuclear system. The identical particles used were negative pions. ${ }^{2}$ Two different acceptances were used for each aystem, centered at $0^{\circ}$ (in the laboratory and the nucleus-aucleus center of mass) and at $45^{\circ}$ in the laboratory, which is approximately $90^{\circ}$ in the center of mas, allowing a search for effects depending on the viewing angle. The data samples for $\mathrm{Fe}+\mathrm{Fe}, \mathrm{Nb}+\mathrm{Nb}$ (fo- both acceptances) and for the $0^{\circ} \mathrm{Ar}+\mathrm{Ar}$, allowed cuts to be made a a function of pion mumentum, allowing a search for effects depending on pion momentum. Equal mase target and projectile were used for all syatems so that the rest frame of the pion source is the rame a the nucleus-nucleus center of mass.

The high statiotica available for thic experiment made neceasary a careful analyais of the spectrometer's effect on the messured correlation function, and a dencription of the

\footnotetext{
"The notation "1.54 - $A \mathrm{GeV}$ " is uned to denole "1.34 GeV per mucleon", the total enerey of the nuclews is then $1.54 \times A$ in units of $\mathrm{GeV}$, where $A$ in the atomic number.

${ }^{2}$ Although pions wese used, any identical bowons could be ween. An analogons effect exints for fermiDiruc purticles.
} 
hardware and software is given, along with the method, used to correct for the effect during the data analysis. For the ame reason, a detailed analysis is made of the method used to generate the background, or reference ample and an existing method was modified to give higher accuracy.

Included here is a discuation of the terminology of two pion correlations as used in relativistic heavy ion phyaica. The word "background" is usually understood to wean everything but the signal decired. In high onercy physics this would mean everything but the pion, since particle identification is a concern. In this experiment, the particle identification is much eader because pions are the only negative particles that are abundantly produced in a nuclear collision at this onergy, and it is common to asume that anything that is negatively charged is a negative pion. For ponitive particles, the only contaminants that ane likely are protone and, for the energy ranges of intereat and the flight path of this spectrometer, it is poucible to distinguish between the pions and protons on the basis of time of atight. Therefore, the "background" sample has come to mean the data sample with only the effect that we are trying to mesure (the Bose-Eirstein correlations) removed. In high energy physics experiments this sumple bas been called the "reference samplen.

The "real" sample is used here to mean the data sample that includes the BoweEinstein correlations and other effects. It is the dats sample that is measured in the experimeat. The correlation function is then the ratio of the "real" sample to the "background" sample, since the real ample jacludes all effects, and the background sample includes all effects except for the Bone-Einstein correlations.

\subsection{Organization}

The organization of this theais is as follows:

Chapter 2 is a review of the experimental and theoretical developments that predate this thexis, both in heavy ion collisions and othor collisions.

Chapter 3 is a discusion of the theories of auclear collisions, explaining the motivations for particle-particle correlation experiments, and a derivation of the Bone-Einstein correlation effect.

Chapter 4 deacribes the apparatun. The locationa of the major components of the 
spectrometer are given so that the acceptance can be reproduced in detail.

Chapter 5 describes the track-finding and particle identification algorithms and their effects on the observed correlation function. Aleo described are a series of cuts made on both the real and the background data so these effects are present in both data samples and hence cancel out when the correlation function is calculated. The chapter concludes by describing bow the momenta of the tracks are calculated from the wire chamber data.

Chapter 6 describes how the background is calculated from the real data using the method of event mixing. A detailed calculation is made to show that, even though the Bose-Einitein correlations are removed through event mixing, the observed but discarded fions affect the momentum epectra of the remaining pions (this is known as residual cosrelation). A method is developed where the events are weighted to approximately remove the residual correlation effect. An jterative procedure is developed where the approximate weights are used to calculate more accurate weights, which can be repeated until the desired accuracy is reached. The chapter concludes with a discussion of the sechniques used to fit the correlation function to the data and the methods used to eatimate the quality of the fit.

Chapter 7 begins by presenting the results of fits to a Monte Carlo simulation of the experiment. The pion pairs were geaerated 20 that their momenta were correlated using the same functional form a was used for the fit correlation function, with sourcesize parameters typical of thone meanured in this experiment. The pion paths in the spectrometer were then simulated, which allowed for the simulation of the spectrometer's acceptance, and for using the usual data analysis stream on the simulated pion tracks. Comparison of this simulated data with the measured data allows determination of which features are due to the spectrometer's acceptance (which the Monte Carlo simulates, and hence these features are present in the Monte Carlo fits) and which features are due to novel physics (which the Monte Carlo lacka, and hence the Monte Carlo fits will lack). Typical fits for the data are shown in detail and the parameters are given for all fits, including fits for which $R_{\perp}=R_{\|}$was forced, which can be compared to earlier result: (where this was assumed). The results of the earlier experiments and the results of this experiment are discunsed.

Chapter 8 discusses future experimental and theoretical needs. 


\subsection{Notation}

In this thesis the convention has been adopted of using math italic lower case letters (e.g., x) for scalars, math italic lower case letters with arrows (e.g., $\vec{x}$ ) for three-vectors, and bold face capital letters (e.g., $X$ ) for four-vectors. In this convention the notation for the four-vector momentum and location are

$$
\begin{aligned}
& \mathbf{P}=(\bar{p}, E) \\
& \mathbf{X}=(\boldsymbol{z}, t)
\end{aligned}
$$

There are some important scalars that have been given capital letters to distinguish them from the run-of-the-mill scalars. These scalars include $R_{\perp}, R_{\|}$(which are cor. relation function parameters), $C_{2}$, also denoted $C_{i j}$, (which in used for the correlation function), $A$ (which is used for normalizations), and $N$ (which is used as the number of events in the experiment).

In this thesis, except for Section 3.3 on effective edge track finding, $Q=(\vec{q}, 90)$, will be used for the momentum-energy difference of the two pions in the event. In Section 5.3, $q$ will be ued for the particle's charge. The notation $\Delta x$, where $x$ is a measured quantity, is used for the uncertainty in $x$. Throughout this thesis the notation $\perp$ (and $\|$ ) will mean the components of a vector perpendicular (and parallel) to the axis through the collision defined by the beam axis. Note that some authors use the same symbolu for other reference axes. 


\section{Chapter 2}

\section{Historical Review}

\subsection{Photon-Photon Correlations}

The earlieat mention of identical particle correlations is that of Hanbury-Brown, Jennicon and Da Gupta in 1952[2]. They discuen the results of using intensity interferometry to measure the angular sizes of radio sources. A later paper[3] presents the theoretical motivation behind the first paper, baed on electromagnetic theory. The deacription of the apparatus in the second paper is:

"Two serials A and B feed two independent receivirs tuned to the same frequency with identical band-pass characteristics. The output of each receiver is rectified in a square-law detector and is fed to a low-frequency band-pass fiter. The outputs $\bar{S}_{1}(t), S_{2}(t)$ of these filters are combined in a correlator ... [emphasis in the original]"

All of the components neceasary for a particle correlation experiment are present - two detectors at different locations counting the number of particles (radio-frequency photons) arriving from an extended source. Here the energy of the photons is fixed; the receiver separation must be varied to find the source size.

Intensity interferometry is baed on the comparison of the intensity of the electromagnetic waves, not the interference of the waves, so the ame theory can be applied to light to produce an interferometry experiment that does not require that the beams of light be brought together to interfere. This is not too surprising since one can reason that 
if intensity interferometry works for radio-frequency photons it should also work for light photons, the only difference being the technical one of particle detection. So HanburyBrown and Twiss adapted their theory for light[4] and used the intensity interferometry of light to measure stellar diameters. They also improved their resolution by the ratio of the wavelengths (although they could no longer detect the same stars as before). It is intereating to note that the mirrore uned in the detectora did not have to be of good optical quality aince one dow not have to resolve the star very well in order to be able to count the piotons coming from it.

Although Hanbury-Brown and Twiss could have used photons in their reasoning, they instead used the fact that photomultiplier tubes are square-law devices, like their radio detectora wase. In 1903, Glauber[5] developed the quantum theory of optical coherence and showed that the Hanbury-Brown-Twias effect was due to photon-photon correlations in incoherent cources. He also showed that for coherent cources ${ }^{3}$ the effect wa absent. This was experimentally verified in 1965 by Armatrong and Smith[6], when they demonstrated that for a solid-atate lawer, driven above the lasing threshold, ${ }^{2}$ the Hanbury-BrownTwise effect was absent. They also found that for the laser driven below the threshold, where it will behave similarly to ordinary thermal cources, the effect is present. For very low drivir 3 currents, the effect begins to disappear, because the probability of two photons being in the system at the same time is small.

\subsection{Particle-Particle Correlations}

A few years after the first photon-photon correlation experiments, Goldhaber, Goldhaber, Lee and Pas[3] rediacovered intensity interferometry while measuring the opening angles of pions produced ia pp collisions in a bubble chamber. ${ }^{3}$ Their analysis interpreted the probability of emiasion at varying opening angles as the effect of the Bowe-Einstein symmetrization of the pionic wave function, and the size of the thermal source jnvolved in the pion emiasion. They calculated the number of pions as a function of the opening angle

\footnotetext{
${ }^{2}$ Here the definition of colverent is (probsbility for a photon in state $i$ and a photon in atute $\left.j\right)^{2}=$ (probability for a photon in stute i) $\times$ (probubility for a photon in otate j). A more seanal defnition and defiaition for higher levele of colverence (involvins more photom) are given in [s].

'The paraneter wod to control the lener in thie experiment wes the curreat through the diode juaction.

'For more detaib concernias particle-particle correlations an [8].
} 
for various source sizes to compare to their data; the concept of a correlation function was not in use at that time.

In the 1970's Kopylov and Podgoretskii[9], Shuryak[10] and Cocconi[11] proposed to analyze the data in terms of a correlation function, where the ratio of the number of particles with Bose-Einstein correlations (or Fermi-Dirac anticorrelations) to the number of particles with out such correlations is plotted as a function of the momentum difference of the particles. This started two discuasions; the first concerns the proper background (or reforence) ample (e.s., the ample without the correlations or anticorrelations), and the second conceras the proper functional form to use for the correlation function that is to be fit to the data. $A$ list of the popular functional forms and conversion factors was given in a recent article[12].

There are several plaunible schemes for calculating the background data sample, some of them are:

1. Use the relative momentum spectrum from the unlike-sign particles (this is done mainly for experiments using pions).

2. Use the relative momentuin spectrum of two like-sign particles coming from different events.

3. Use the relative momentum spectrum of two unlike-sign pions coming from different events.

4. Use the relative momentum spectrum from theoretical calculations that include all of the collision dynamics except for the Bose-Einstein correlations.

The first method has problems with resonances contributing to the unlike-sign particle spectrum differently than to the like-sign particle spectrum. This method is commonly used in high enersy experiments where purticles of both charge are detected, the effect of resonances can be cut out, and concervation of energy and momentum is a concern. The second method ha problems with momentum and energy conservation violations, and residual correlationst appearing in the peendo-events. This method has been ueed in heavy ion collitions where the momentum-energy violations generated are small since the

\footnotetext{
'See [1] or Chaptat 8, this thain.
} 
pions carry oaly a small part of the total momentum and energy of the nuclear collision. The third method combines the first and second methods. The role of reconances will he different in this method than in the first method, a possible advantage. The last method has the difficulty of requiring that almost everything muat be knowin about tite collisionis dyramica before the experiment is done. This was the method used in [7], in a form involving integration over many variables.

Work on Pp two pion correlations led to additional papera $[13,14]$ that provided increacingly detalled analyais of the data and uncertainties. These papers serve to point out how difficult it is to generate a good background (or reference) data net. A later paper[15] showed, by Monte Carlo simulations, that one of the methode uned to generate the background data sets (that of shuming the components of the momenta transverse to the beam) can induce correlations in the background data, which will obscure the correlations in the measured data.

The Mark II collaboration[16, 17, 18, 19] has studied Bose-Einstein correlations at SPEAR and PEP finding only small variations in the parameter $\beta$, where the functional form for the correlation function was $C_{2}(Q)=1+\alpha e^{-\phi Q^{2}}$, from 4-7 GeV (SPEAR) to $29 \mathrm{GeV}$ (PEP). In this paremeterization, $Q$ iü iüe invariani momentum difierence $(Q=\sqrt{Q 4}), \beta$ is the size of the correlation and $\alpha$ is the strength of the correlation (analogous to $\lambda$ in Chapter 3 of this theais). A recent paper[20] has proposed that because of the correlation between the momentum of the particle and its emission point in this type of collision, the source size as measured will reflect the momentum-position correlation effect! and will not show the size of the pion emitting source. However, the apparent source size provides information on particle production in the collinion proceas[20]. ${ }^{5}$

\subsection{Particle Correlations in Nuclear Collisions}

In suclear colliaions, there is a length ecale present other than that induced by the position-momenium correlation, that of the nuclear diameter. ${ }^{8}$ It is thought that the source size will reflect the motion of the hot auclear matter formed in the early stages of the collinion. It was hoped that the pion source size would reveal what the nuclear matter

\footnotetext{
'For more detsile concerning high energy inteructions me [18].

- For mose detcily conceraing heary ion collisions we [21].
} 
density was at "freeze out" - the moment that nucleons were far enough apart and cool enough that the interaction phase of the collixion could be considered to be over. Another area of interest was the hoped-for ability to determine if coherent emission of pions was taking place in the collision (pion condensation), producing the pion equivalent of a laser.

$A$ series of experiments was conducted at the streamer chamber at the LBL Bevalac accelerator $[22,29,24,25]$ using $1.8 \cdot \mathrm{A} \mathrm{GeV}{ }^{40} \mathrm{Ar}+\mathrm{BaI}_{2}, 1.8 \cdot \mathrm{A} \mathrm{GeV}{ }^{40} \mathrm{Ar}+\mathrm{Pb}_{3} \mathrm{O}_{4}$, 1.5 $\cdot \mathrm{A} \mathrm{GeV}{ }^{* 0} \mathrm{Ar}+\mathrm{KCl}, 1.2 \cdot A \mathrm{GeV}{ }^{40} \mathrm{Ar}+\mathrm{KCl}$, and $1.8 \cdot \mathrm{A} \mathrm{GeV}{ }^{40} \mathrm{Ar}+\mathrm{Pb}$. While the $4 \pi$ detector allowed detection of many pions in each event, improving the statiatica in the experimente, most of the pions were at lary relative momentum differences, where the correlation efiect in minimal.

In the 1980's Zajc et al.[1] conducted several experiments at the LBL Bevaluc uning a magnetic apectrometer and two beam-target combinations (1.8 $\cdot \mathrm{A} \mathrm{GeV}{ }^{40} \mathrm{Ar}+\mathrm{KCl}$ and 1.8 - A GeV Ne + NaF). These experiments allowed good statistics at low values of the momentum difierence, while introducing the complication that the background was not as well determined $a$ in the $4 \pi$ streamer chamber experiment and also contained larger residual correlations. This work uses a modified form of the spectrometer used in those experiments.

In 1986, Beavis et al.[25] (see also [26] and [22]) measured the pion source size as a function of the negative pion multiplicity (denoted here $N_{z^{-}}$) of the nuclear collition. The reaction studied was $1.8 \cdot A \mathrm{GeV} A r+P b$. The reaults ware, for $2 \leq N_{\pi}-\leq 7, R=3.59$ $\pm 0.71 \mathrm{fm}$ and, for $11 \leq N_{\mathrm{R}} \leq \leq 20, R=5.97 \pm 0.52 \mathrm{fm}$, where the function fit is the single radius parameter fit a discuesed in Section 7.? fad Chapter 3. This showed that for this asymmetric system, the measured source aize depends on the pion multiplicity and, therefore, the centrality of the collision.

In that same year, Humanic m.sde[27] a comparison of the source size of pions generated by the nuclear collision simulation code CASCADE and the data of Zajc et al.[1] and Beavis et al.[23, 24]. While the agreement wa generally acceptable, the imall values of the parameter $\lambda$ obeerved were not predicted by CASCADs. ${ }^{\top}$

Other experiments at the Bevalac include the Plastic Ball[28], where a fast/slow scintillation combination was used to provide $d E / d X$ and $E_{\text {fotal }}$ information for $2 \sim 4 \pi$

\footnotetext{
'The data from [1] are included is Table 7.4 and the prediction from [27] are incladed in Table 8.2.
} 
solid angle. Unfortunately the granularity of the detector (and possibly other effects) did not allow 2 very good measurement of the correlation function. The systems used were $650 \cdot \mathrm{A} \mathrm{MeV} \mathrm{Nb}+\mathrm{Nb}$ and $650 \cdot \mathrm{A} \mathrm{MeV} \mathrm{Au}+\mathrm{Au}$. In the HISS (Heavy Ion Spectrometer System) facility, the correlation function was measured using a magnetic spectrometer and drift chambers for $\mathrm{Ar}+\mathrm{KCl}$ (making this projectile-target combination one of the most popular for measuring the corralation function). These data are still under analysis as of the writias of this thecis[20]. 


\section{Chapter 3}

\section{Motivation and Theory}

\subsection{Motivation}

Virtually all the computer code[30] that are being und to predict the properties of nuclear collicions at the present time are transport codes of one.form or another. Since it is dearable to be able to predici the propertien of the collinion as a function of the impact parameter, almont all of the codea have the ability to locate the nucleons in space. Since pion production is part of the nuclear colliaion, these codes are capable of predicting the deasity of the pion courcen in space. The codee that allow ncaltering of the pions generally allow one to modify "sources" to mean the location of the lat acattering of the pion before it leaves the collision. So, if nuclear tranuport codes are to be able to correctly predict quantities that reflect the nucleon dintributions during the course of the nuclear collision then they must be able to correctly predict the pion source size.

The difficulty is in generating the theoretical source size to compare to the measured sizes. This has been done in the case of $1.70 \cdot \mathrm{A} \mathrm{GeV}{ }^{56} \mathrm{Fe}+\mathrm{Fe}$, using the Cascade model[27, 32], which is part of the data reported here. In principle, predicted source sizes (in the parameterization of the correlation function used in this theais) for all of the computer codes in use, could be generated for all of the reactions studied here. Because of the large number of codes and unmanageably large amounts of CPU time that would be required, generating source sizes for all the computer transport codea was not attempted

\footnotetext{
'Zajc[31] gives procedurai for aymmetrining large numbers of Monte Curlo piom from the aume collision, bat one atill hes to generete the piones.
} 
in this thesis but was left for those who have developed (or will develop) the cories.

\subsection{Derivation of Bose-Einstein Correlations}

Suppose ${ }^{2}$ that a pion is emitted at the (four-vector) position $R_{1}$, a second pion is emitted at $\mathbf{R}_{2}$ and the momanta of the pions are meaured by two detectorn, giving momentum $\mathbf{P}_{1}$ at position $\mathbf{X}_{1}$ and $\mathbf{P}_{2}$ at $\mathbf{X}_{2}$. Alauming that the pions aro indiatinguiohable (includins knowing where the pions came from) then, alnce the plons are bosons, the wave function describing the plons must be aymmetric under interchange of the particle labela, that is under interchange of $\mathbf{R}_{1}$ and $\mathbf{R}_{2}$ in the wave function. This is equivalent to allowing elther of the particles to have come from $\mathbf{R}_{1}$ or $\mathbf{R}_{2}$.

Anuming further that the particles may be deacribed by plane waves, then the pion wave function is (in units such that $h=c=1$ )

$$
\checkmark\left(\mathbf{P}_{1}, \mathbf{P}_{2}\right) \propto \frac{1}{\sqrt{2}}\left(e^{i P_{1}\left(X_{1}-R_{1}\right)} e^{i P_{2}\left(X_{2}-\mathbf{R}_{2}\right)}+e^{i P_{1}\left(X_{1}-R_{2}\right)} e^{i P_{2}\left(X_{2}-R_{2}\right)}\right)
$$

where the usual definition for the dot product of four-vectors is used

$$
\boldsymbol{P}_{i} \mathbf{X}_{i}=\overrightarrow{p_{i}} \cdot \vec{z}_{i}-E_{i} t_{i} \text {. }
$$

The probability of a given state (relative to the same state without the Bose-Einstein symmetrization) is $\cdot+$, which is

$$
\left|\mathbf{P}\left(\mathbf{P}_{1}, \mathbf{P}_{2}\right)\right|^{2} \propto 1+\frac{1}{2}\left(e^{i\left(R_{1}-R_{2}\right)\left(P_{1}-P_{2}\right)}+e^{-i\left(R_{1}-R_{2}\right)\left(P_{1}-P_{2}\right)}\right)
$$

Assuming that in a heavy ion collision all the pion cource act incoherently, ${ }^{3}$ and are distributed according to the distribution function $p(\mathcal{Z}, t)$, then the two pion counting rate is

$$
\mathcal{P}\left(\mathbf{P}_{1}, \mathbf{P}_{2}\right) \propto \iint \rho\left(\vec{f}_{1}, t_{1}\right) \rho\left(\vec{F}_{2}, t_{2}\right)\left|\Psi\left(\mathbf{P}_{1}, \mathbf{P}_{2}\right)\right|^{2} d^{4} \mathbf{R}_{1} d^{4} \mathbf{R}_{2} .
$$

The integrals over poation are to be taken over all space. - the timie integraln, the time between th suclear collixion and detection of the particles is anumed to be sufficiently long that the limits may be extended ' all times with negligible error.

\footnotetext{
'Thin derivation followe that of [33].

${ }^{3}$ Here iscolvereatly is defined a (probability of a pion in tute $i$ and a pion in stute $j$ ) = (probability of a pion in reate $i$ ) $\times$ (probability of a pion in atate $j$ ), where the Bone-Einctein symmetrization is to be concidered shut of. See Section 3.5 for more detsil.
} 
The corresponding two pion counting rate without the Bose-Einstein symmetrization is

$$
\mathcal{P}^{\prime}\left(\mathbf{P}_{1}, \mathbf{P}_{2}\right) \propto \iint \rho\left(\vec{r}_{1}, \hat{t}_{1}\right) \rho\left(\vec{r}_{2}, t_{2}\right) d^{4} \mathbb{R}_{1} d^{4} \mathbf{R}_{2} .
$$

Asuming the normalization

$$
\int \rho\left(F_{1}, t_{1}\right) d^{4} \mathbf{R}_{1}=1
$$

then Eq. 3.4, becomes

$$
\begin{aligned}
P^{\prime}\left(P_{1}, P_{2}\right) & \propto \int \rho\left(f_{1}, t_{1}\right) d^{d} R_{1} \int \rho\left(f_{2}, t_{2}\right) d^{d} R_{2} \\
& \propto 1 .
\end{aligned}
$$

With a little algebra, the equation for the rate with the Bose-Einutein aymmetrization included, Eq. 3.3, becomea

$$
\mathcal{P}\left(\mathbf{P}_{1}, \mathbf{P}_{2}\right) \propto 1+\left|\vec{p}\left(\vec{\varphi}, q_{0}\right)\right|^{2},
$$

so that the ratio of the two pion counting rate with Bowe-Einstein symmetrization to the rate without is

$$
\begin{aligned}
C_{3}\left(\mathbf{P}_{1}, \mathbf{P}_{2}\right) & =\frac{\mathcal{P}\left(\mathbf{P}_{2}, \mathbf{P}_{2}\right)}{\mathcal{P}^{\prime}\left(\mathbf{P}_{1}, \mathbf{P}_{2}\right)} \\
& =1+\left|\vec{P}\left(\vec{q}, q_{0}\right)\right|^{2},
\end{aligned}
$$

where

$$
\begin{aligned}
\vec{q} & =\overrightarrow{p_{2}}-\overrightarrow{p_{1}} \\
q_{0} & =\left|E_{2}-E_{1}\right| \\
\vec{p}\left(\vec{q}, q_{0}\right) & =\iint e^{i\left(p \cdot t+\omega^{t}\right)} \rho(\vec{x}, t) d^{3} \vec{x} d t \\
& =\text { the Fourier tranoform of } \rho(\bar{x}, t) .
\end{aligned}
$$

Some of the propertice of the probability are: Firat, the symmetry under interchange of $P_{1}$ and $P_{2}$ in Eq. 3.3 implies that $\vec{p}\left(\vec{q}, q_{0}\right)$ is an even function in both $\vec{q}$ and 90, so that the absolute value in Eq. 3.10 is not strictly neceasary, and the order of $\overrightarrow{p_{1}}$ and $\vec{p}_{2}$ in Eq. 3.9 is not important. Second, the probability of emission is increased relative to the probability of emission of identifiatie particle for small values of $\vec{q}$ and $q 0$. Third, for Fermions the plus siga in Eq. 3.8 becomes 2 minus sign and the emission at small $Q$ 
is suppresed. Last, it is possible to justify Eq. 3.8 by saying that for $\Delta x \cdot \Delta p \leq \hbar=1$ and $\Delta E \cdot \Delta t \leq \hbar$ (where $\Delta x=\left|r_{1}-r_{2}\right|, \Delta p=\left|p_{1}-p_{2}\right|$ and so on) then the uncertainty principle allows the wave functions to overlap, and hence to interfere.

\subsection{Correlation Function Used in the Fit}

Genorally it is not posible to invert the Fourier transform (particularly aince whet is measured is the square of the abeolute value of the Fourier traufform), ac a source density function with soveral fres parameters is asumed. Its Fourier traseforco is then calculated, and the free parameters are adjusted for beat agreement with the data. The choice of the parameterization uned is made according to the phyvics is be explored.

Fourier transforms have the general property that large scaly: features traneform to small scale features. For example, the overall normalization of the number of pios emitters, 2 property of the integral of $\rho(\vec{x}, t)$ over all spase, tranaforms to the property that the intercept of the correlation function at $Q=0$ is $C_{2}(Q \rightarrow 0)=2$ (this is best sean by setting $P_{1}=P_{2}$ in Eq. 3.2 and then substisutiag the result into Eq. 3.3). Because the finite amount of data that can be taken in a realiatic experitseat restricts the resolution of the correlation function, which is roughly the Fourier transform of the pion source density, only the larger features of the pinn source deanity are well determined. These are the lifetime and the radius parameters in the two directions that the spectrometer can determine, the radius parameter parallel to the beam and the radius perpendicular to the beam (the spectrumeter cannot determine the impact plane).

In this experiment the source density $p(\bar{x}, t)$ is assumed to be the Gaussian

$$
\rho(x, t) \propto e^{-\left(\frac{t_{1}}{x_{1}}\right)^{2}-\left(\frac{\pi_{1}}{x_{1}}\right)^{2}-\left(\frac{1}{t}\right)^{2}}
$$

Where the notations $\perp$ (and II) mean perpendicular (and parallel) to the axis through the collision defined by the beam axis. The parameters $R_{\perp}, R_{\|}$and $\tau$ are the two radius parametert and the lifetime parameter. The Gaussian cource deasity is choser because the the transform is particularly simple to calculate. Note that other paramoterizations will yield similar forms for the correlation funetion, but the parameters may differ by a multiplicative factor. When comparing data it is essential to check the parameterizations used. 
Assuming this source density the probability can be calculated 2 .

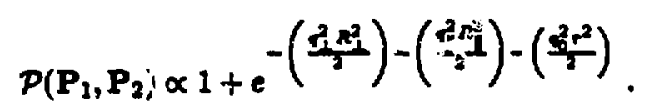

A number of aunumptions have been made in deriving these equations and what follow! is a discusaion some of the important ons. It has been shown that replacine plane wavem with Coulomb waves (but retaining the Gaussian acurce) changes the result by $<0.1 \%$ in the region of $f$ important for this effect[34]. Changing from a Gausian cource to $x$ uniform deavity spherical source (while asuming plune waves) shanges the value of the correlation function by less than $2 \%$ if $R_{\text {Gausian }}=1.52 \cdot R_{\text {uniform }}[7]$. Sn it is expected that a large variety of sources can be fit with the Gaussian parameterization.

The a priori ssumption of incoherent sources shouid be relaxed to allow it to be verified by experiment. If coherent sonrces were to exist, then the efiscts of the BoseEinstein correlations could be increased or reduced or eliminated. This will be discussed further in Section 3.5. Further, if particle misidentification occurs, then the supposed identical particle sample will include a number of pairs of particles of different types which will not interfere. This would leasen the mesesured effect. To allow for these (and other poutibilitiea) a parameter $\lambda$ was introduced $[35,14]$ giving us the correiation function that was fit in this experimeut

$$
C_{2}\left(P_{1}, P_{2}\right)=1+\lambda e^{-\left(\frac{2 k_{1}^{2}}{2}\right)-\left(\frac{A_{1}^{2}}{2}\right)-\left(\frac{d^{2} x^{2}}{2}\right)} .
$$

The correlation function is usisally written with a mubscript 2 to indicate that this is a second order (two particle) correlation effect. Further, the sormalization assumed is such that for large values of $\vec{q}, q_{0}$ the correlation function is 1 .

There has been an implicit assumption in the choice of the functional form for the source density $p(\bar{x}, t)$ since it anumes that $p$ has no momentum dependence. The choice for the definition of the experimentally determined correlation function (Eg. 3.15) will allow for a momentum dependence as long as it is the ame at all locations, because it will cancel out. Howevar, the momentum dependence cannot be a function of the emission point. Such a momentum dependence could be envisioned as arising from irotropic enission in the local reat frame of al extended, expanding source. Pratt[36] has shown that such a 
source would give an apparent source size that would decrease as a function of the pion energy.

\subsection{Experimental Correlation Function}

In the experiment the correlation function is defined to be the ratio of the two particle cross section to the product of two single particle cross sections with a normalization fuctor

$$
C_{2}\left(P_{1}, P_{2}\right)=D \frac{\frac{d p_{1}^{3} d p_{2}^{3}}{d p^{3} g}}{d p_{1}^{3} \frac{d^{3} q}{d p_{2}^{3}}}
$$

where $D$ is a normalization conatant, $d \sigma / d p_{1}^{3} d p_{3}^{3}$, the two-pion inclusive crons section, and $d^{3} \sigma / d p^{3}$, the singlo-pion inclusive cross section. While it is in principle possible to meanure the various croms actions separately, that is not usually done. Usually, in nuclear collision experiments, the single pion crow sections (the background) are estimated from relstive momentum spectrum of like-sign pions from different svents (event mixing).

The reasons for using event mixing are outlined below:

Using mixing of unlike-sign pions has the difficulty that the spectrometer can only detect one sign of particle at a time. Anuming that trisgering difficulties and the proton contamination could be dealt with, twice the beam time would be needed to do the experiment. There is also the theoretical uncertainty due to the different production rates of $\pi^{+}$and $\pi^{-}$and the different distributions of the protons and the neutrons in the nucleus.

Using theoretica methods to generate the background has the difficulty that nuclear collisions are not understood well enough to predict what the two-particle momentum spectra would be in the abeence of the Bone-Einstein effects. There is also the objection of using the model that is to be teated to generate the data that are supposed to be testing the model.

Simply meanuing the single pion momentum spectrum has the difficulty that the number of pions produced in the nuclear collision depends on the impact parameter. Therefore, the requirement of two pions in the spectrometer biases the two-pion trigger towards more central collisions than the oue-pion trigger[1]. The one-pion triggered data 
would then come from a different class of nuclear collision than the two-pion data and would not be a valid background sample. The only way to properly reproduce the bias is to sequire that the track-finding routines find two tracks and ignore one - which is the event-mixing technique.

Use of the event-mixing technique requires calculation of the effects of the observed but discarded pions in each of the events that are mixed to sorm the pseudo-event. This will be discussed in detail in Chapter 6.

\subsection{Coherence and the $\lambda$ Parameter}

The parameter $\lambda$ in Eq. 3.14 ha beea given the interpretation that if $\lambda=1$ then the source is fully incohereat, and if $\lambda=0$ then the source is fully coherent. Before commenting on this interpretation, it is necessary to define both $\lambda$ and "coherent".

The parameter $\lambda$ is defined in Eq. 3.14 but there are two ponsible interpretations. . The first interpretation is the value of $\lambda$ that would be obtained in the limit of infinite information about a single nuclear collision, which will be denoted $\lambda_{\text {trus. }}$ The second is the value of $\lambda$ that would be obtained from applying the event-mixing technique to a data sample of arbitrarily large vize, which will be denoted $\lambda_{\text {obo }}$.

In elementary optice "coherent" is defined a knowledge of the electric field at one location allows one to predict the electric field at another location (in the radiation field). This definition makes no mention of the number of photons present, and applies even if one photon at a time passes through the apparatus. Glauber[5] termed this "first order" coherence and pointed out that this level of information wa insufficient to determine the outcome of a correlation experiment, which requires two photons.

Definitions of second order coherence are based on properties of two photons. A simplified form of Glauber's definition of second order coherence[5] is

(probability of a photon in state $i$ and a photon in state $j)^{2}=$ (probability of

a photon in state $i$ ) $\times$ (probability of a photon in state $j$ )

The definition of incoherence then is

(probability of a photon in state $i$ and a photon in state $j$ ) $=$ (probability of

a photon in state $i) \times$ (probability of 2 photon in state $j$ ) 
Although photons are used here, any particle could be used. In particular, for a two pion correlation experiment one would replace "photon" with "pion".

An example of a second order coherent source is a laser. The important feature of the laser for second order coherence is the amplification technique, not the monochrornatic nature of the light. Amplification by stimulated emission results in many photons leaving the laser in the same atate. Therefore, if there is a photon in a given state it is extremely likely that there in a second photon in the same atate, and this state is the only state populated by photons. Taking extremaly likely to be $100 \%$, the probabilities (a a function of the photon momentum) are

$$
\begin{aligned}
\mathcal{P}\left(\mathbf{P}_{1}, \mathbf{P}_{2}\right) & =\mathcal{P}\left(\mathbf{P}_{1}\right) \\
& =\mathcal{P}\left(\mathbf{P}_{2}\right)
\end{aligned}
$$

and the laser is second order coherent. Further, $C_{2}\left(P_{1}, P_{2}\right)=1$ and $\lambda_{\text {trus }}=0$.

So if "pion lasers" are the sole source of pions in the nuclear collision then $\lambda_{\text {trwe }}=0$. If there are coherent and incoherent sources in the collision then $\lambda_{\text {true }} \leq 1$. Simple argument: show that if the single-particle momentum spectra are the same for the coherent and incoherent sources then

$$
\lambda_{\text {true }}=\frac{1}{1+J}
$$

where $f$ is the ratio of the number of coherent pion pairs to the number of incoherent pion pairs.

The effect of pion lasers on $\lambda_{\text {obe }}$ depends strongly on the event-mixing technique, and underlines the assumption (often not stated) that all events must have the same momentum distribution for the technique to appiy. Imagine the simple case where a pion laser is the sole source of pions in all of the nuclear collisions in the experiment. If the pion laser always has the same orientation, then event mixing produces the true background and $\lambda_{\text {obe }}=0$. If the pion laser changes orientation randomly from one event to the next (for example, the first $\Delta(1236)$ to decay triggers the laser and determines the direction) then the real spectrum will be a $\delta$-function. The background calculation will mix pions of random orientation, and hence the spectrum will be fat. The ratio of the two will be a $\delta$-function and $\lambda_{\text {obo }}$ will be arbitrarily large.

\footnotetext{
"Note that all photono leavias the lever in the ame atate in nymmetric under interchange of particle labels and the symmetrisation imponed in Section 3.2 doen not apply.
} 
Pion lasers are not the only mechanism that can induce second order correlations. Decay of resonances (where conservation of momentum is a factor), and dynamical correlations (where $\rho$ depends on $\bar{p}$ through the dynamics of the collision) can influence the value of $\lambda[37]$. 


\section{Chapter 4}

\section{Apparatus}

\subsection{The Bevalac Accelerator}

The heavy ion beams for this experiment were provided by the Bevalac accelerator at Lawrence Berkeley Laboratory. They were transported to the experiment using beam line 30-2, which consists of magnetic dipole and quadrapole magnets. The parameters for the beame ured are given in Table 4.1 .

\begin{tabular}{|c|c|c|c|c|}
\hline Ion & Energy & Typical Intensity & Beam Spot Size & Year \\
\hline \hline${ }^{00} \mathrm{Ar}$ & $1.82 \cdot \mathrm{A} \mathrm{GeV}$ & $1 \cdot 10^{8}$ Ions $/ \mathrm{spill}$ & $1 \mathrm{~cm} \times 1.6 \mathrm{~cm}$ & 1986 \\
${ }^{36} \mathrm{Fe}$ & $1.70 \cdot \mathrm{A} \mathrm{GeV}$ & $1 \cdot 10^{7}$ Ions/spill & $1 \mathrm{~cm} \times 6 \mathrm{~cm}$ & 1982 \\
${ }^{93} \mathrm{Nb}$ & $1.54 \cdot \mathrm{A} \mathrm{GeV}$ & $2 \cdot 10^{7}$ Ions/spill & $1 \mathrm{~cm} \times 1 \mathrm{~cm}$ & 1986 \\
\hline
\end{tabular}

Table 4.1: Beam parameters for the runs.

\subsection{The Janus Spectrometer}

The Janus spectrometer, as shown in Figs. 4.1 and 4.2, consists of two dipole magnets, a "C" magnet aear the target, and a window-frane magnet that is called the "Janus" magnet (after the Roman god). Between the $\mathrm{C}$ magnet and the Janus magnet are two wire chambers, and after the Janus magnet are two more wire chambers. The firat pair of chambers define the in-going (to Janus) vector, and the second pair of chambers define the out-going (from Janus) vector. The dellection in Janus gives the particles' momentum. 


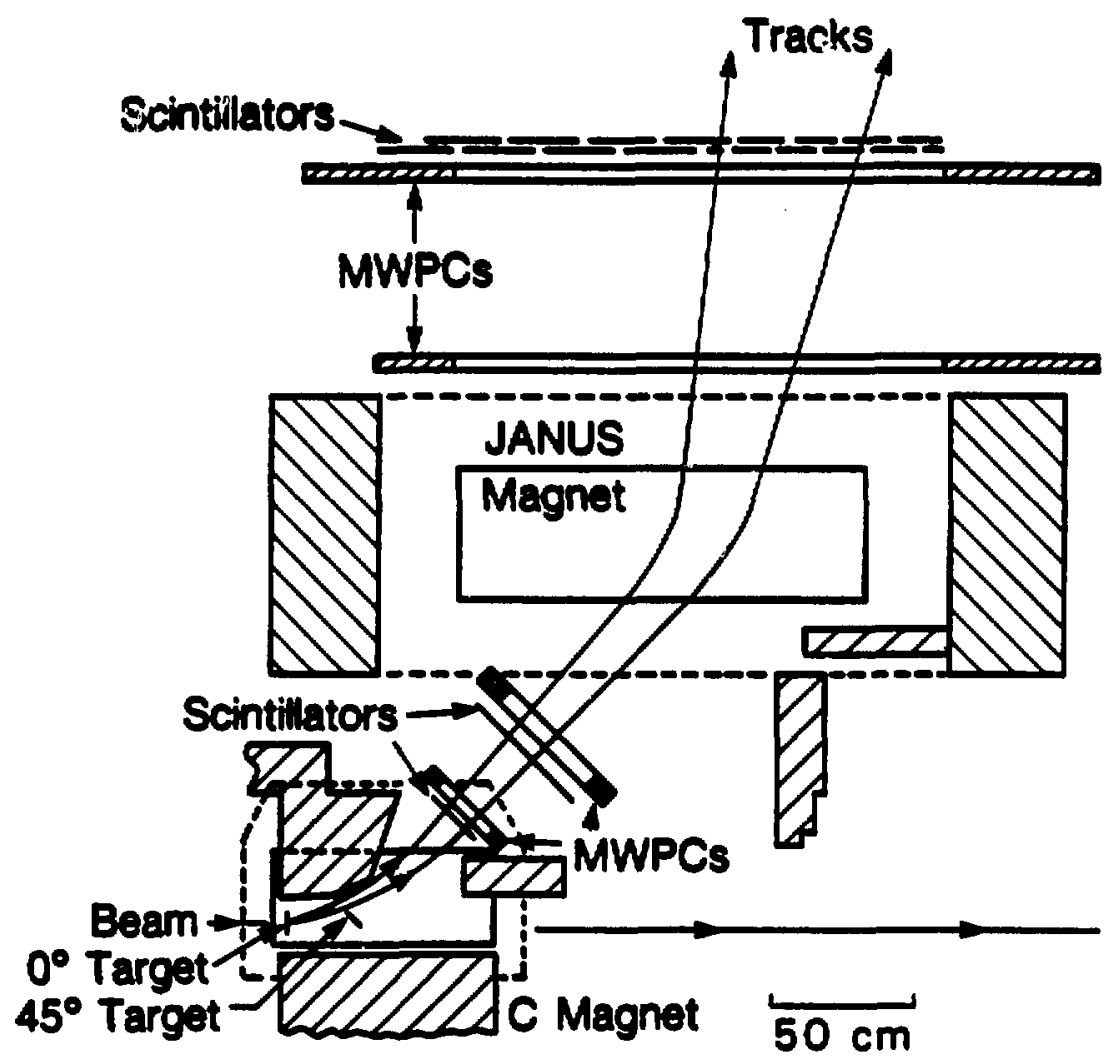

Figure 4.1: The Janu spectrometer in the plan view (1982). 


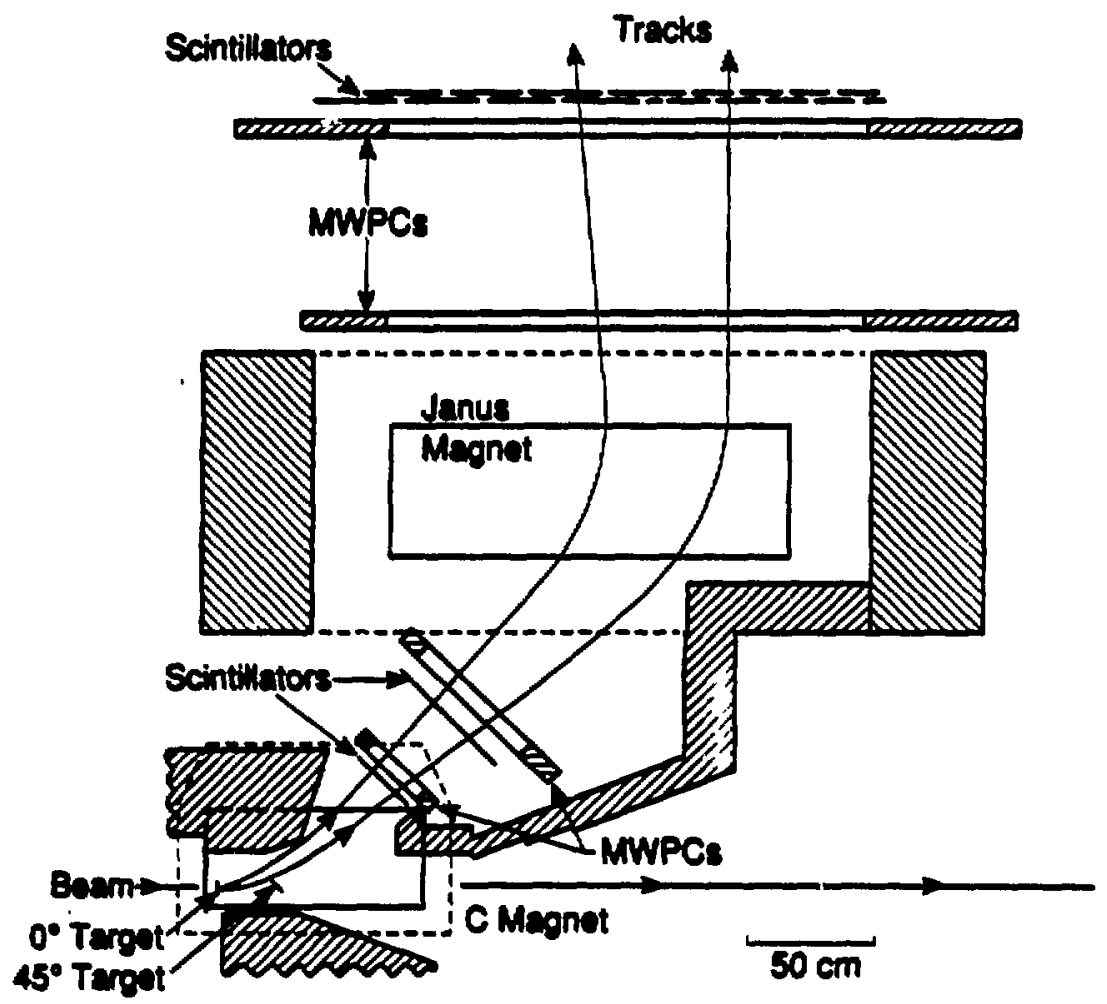

Figure 4.2: The Janus spectrometer in the plan viow (1986). The spectrometer was modified between the ruas by adding load walls to protect the socond wire chamber from s-rays produced by the baem interacting with the air downotream of the target, and replacing the second wire chamber. 
There are also a number of scintillation counters for triggering, time of flight determination and energy-loss measurement. Figure 4.1 shows the spectrometer as run in 1982 and Figure 4.2 shows the spectrometer as run in 1986. The spectrometer was modified between the runs by adding lead walls to protect the second wire chamber from $\delta$-rays produced by the beam interacting with the air downatream of the target, and replacing the second wire chamber.

The detailed deacription of the components will be given in approximate order of diatance from the exit window of the beam line. The $\mathrm{C}$ magnet has a pole tip size of $91.4 \mathrm{~cm}$ by $40.6 \mathrm{~cm}$ and a pole gap of $20.0 \mathrm{~cm}$. In this configuration the magnet can give fielde in the range of about $1 \mathrm{KG}$ to about $14 \mathrm{KG}$. The magnet wa mapped after the Fe data were taken using a feld mapper from the Magnetic Meacurements group. The field mapper measured the voltages acrose three orthogonal search coils a they were moved through the field volume. The data from the mapper were procenad on the sevax VAX-11/780. The field mapper and proceating techniques used are similar to those used for the Janus magnet in an earlier experiment[1].

\begin{tabular}{|c|c|c|c|c|c|}
\hline & \multicolumn{3}{|c|}{ Target } & \multicolumn{2}{|c|}{ Magnet fieldo } \\
\hline Set-up & Material & Ponition & ancle & C magnet & Janue magnet \\
\hline $\operatorname{Ar} 0^{\circ}$ & $\mathbf{K C l}$ & $26.0 \mathrm{~cm}$ & $90^{\circ}$ & $14.6 \mathrm{KG}$ & $12.0 \mathrm{KG}$ \\
\hline Ar $4^{\circ}$ & $\mathrm{KCl}$ & $2.8 \mathrm{~cm}$ & $45^{\circ}$ & $1.7 \mathrm{KG}$ & $9.0 \mathrm{KG}$ \\
\hline Fe $0^{\circ}$ & Stainless Steel & $26.0 \mathrm{~cm}$ & $90^{\circ}$ & $14.2 \mathrm{KG}$ & $11.4 \mathrm{KG}$ \\
\hline $\mathrm{Fe} 45^{\circ}$ & Fe & $0.0 \mathrm{~cm}$ & $45^{\circ}$ & $0.0 \mathrm{KG}$ & $8.6 \mathrm{KG}$ \\
\hline $\mathrm{Nb} 0^{\circ}$ & $\mathbf{N b}$ & $26.0 \mathrm{~cm}$ & $90^{\circ}$ & $13.0 \mathrm{KG}$ & $10.8 \mathrm{KG}$ \\
\hline $\mathrm{Nb} 45^{\circ}$ & $\mathrm{Nb}$ & $0.0 \mathrm{~cm}$ & $45^{\circ}$ & $1.5 \mathrm{KG}$ & $8.5 \mathrm{KG}$ \\
\hline
\end{tabular}

Table 4.2: Spectrometer parameters for the set-ups used.

The target materials used in this experiment are given in Table 4.2. The targets were placed in a target holder that rested on the lower pole tip of the $\mathbf{C}$ magnet. This places a reatriction on the choice of target in that it cannot be magnetic if the $\mathrm{C}$ magnet is used. For this reason the target used in the $0^{\circ}$ Fe runs was \#304 stainless steel. The composition of the stainjeas steel target (a given by the supplier, Cantle Metals - A. M. Castie \& $\mathrm{Co}$.) is given in Table 4.3. For the Ar run $2 \mathrm{KCl}$ target was used to avoid the 
necessity of using a cryogenic target. Since the properties measured depend on the mass number of the colliding species the slight difference in mass was not important. Targets 0.5 to $1.0 \mathrm{gm} / \mathrm{cm}^{2}$ thick were uned in all cases as a compromise between the data-taking rate and the multiple scattering in the target. At this thicknes, and for the materials used, the multiple acattering in the target is approximately equal to that in the first two wire chambere and countere.

\begin{tabular}{|c|r|}
\hline Eloment & Amount \\
\hline $\mathrm{F}$ & $70.9 \%$ \\
$\mathrm{Cr}$ & $18.5 \%$ \\
$\mathrm{Mi}$ & $8.5 \%$ \\
$\mathrm{Mn}$ & $1.6 \%$ \\
$\mathrm{Si}$ & $0.5 \%$ \\
\hline
\end{tabular}

Table 4.3: Stainleas steel target componition. Percentages are by weight.

The target location and orientation ured depends on the desired acceptance, as do the magnetic fields. This information is contained in Table 4.2. The origin for the coordinate syatem is taken as the $45^{\circ}$ target poaition and ponitive is upotream. The angle is the angle between the target plane and the beam direction, $45^{\circ}$ being such that the target is facing the Janus magnet.

The first scintillation counter (S1) is $30.3 \mathrm{~cm}$ by $15.2 \mathrm{~cm}$ and $0.18 \mathrm{~cm}$ thick. The photomultiplier tube used is an XP2020 with an active base suitable for high count-rate environments.

The firot wire chamber follows S1. The active area is $30.5 \mathrm{~cm}$ by $15.25 \mathrm{~cm}$ and contains about 400 wires. The wire spacing is $0.2 \mathrm{~cm}$ and the distance between sense planes is $1.4 \mathrm{~cm}$. The first plane of wires (closent to the target) is angled at $45^{\circ}$, the second plane is strung at $90^{\circ}$ (vertically), and the last plane is atrung at $0^{\circ}$ (horizontally). It is read out by a wire chamber readout aystem designed and built at LBL[38].

The second scintillation counter is actually two countern side-by-side (called S2A and S2B - S2A is upstream). The active area is $55.9 \mathrm{~cm}$ by $19.1 \mathrm{~cm}$ and is equally split by the two counters, which overlap slightly. The counters are $0.18 \mathrm{~cm}$ thick. These counters are each viewed by an RCA 8575 phototube on a standard LBL base. These counters are 
used to determine the start of the time of flight since it was felt that the lower counting rates in $\mathbf{S 2}$ than in $\mathrm{S1}$ would allow a better determination of the start of the event.

The second wire chamber follows the S2 counters. During the Fe runs, the wire chamber used was $52 \mathrm{~cm}$ by $19.4 \mathrm{~cm}$ in active area. The full height was $40 \mathrm{~cm}$, but some of the horisontal wires were not used. The order of the planes in this wire chamber was, the first plane was $0^{\circ}$, the excond was $90^{\circ}$ and the last plane was $-45^{\circ}$. The negative angle means that the upatream end of the wire is lower than the downotream end. The wire spacing was $0.2 \mathrm{~cm}$ for $0^{\circ}$ and the $90^{\circ}$ planes and $0.3 \mathrm{~cm}$ for the $-45^{\circ}$ plene. Between the frat and second sense planes the upacins was $1.2 \mathrm{~cm}$, and between the second and the third cenes planes the opecing was $2.2 \mathrm{~cm}$. The wire chamber contained about 600 wires, and was and out by the LBL wire-chamber read-out syatem.

For the Ar/Nb runs a diferent sacond wire chamber was used. $\quad$ The new wire chamber had an active area of $37.6 \mathrm{~cm}$ by $19.2 \mathrm{~cm}$. The full height of the wire chamber is $32.0 \mathrm{~cm}$ but, again, not all of the horizontal wires were used. The order of the planes in this chamber is $0^{\circ}, 90^{\circ}$ and then $-45^{\circ}$. The wire spacing is $0.2 \mathrm{~cm}$ in all sense planes and the spacing between the sense planes is $1.4 \mathrm{~cm}$, This chamber contains about 700 active wires, and it is read out by a LeCroy PCOS3 wire chamber readout aystem.

The Janus magaet follows wire chambar two. This magaet has a pole tip aize of $167.6 \mathrm{~cm}$ by $55.9 \mathrm{~cm}$, and a pole gap of $21.4 \mathrm{~cm}$. The magnetic field of the Janus magnet was mapped for an earlier experiment[1].

Following the Janus magnet are wire chambers three and four. There wire chambers are electrically identical, and the active areas are $200 \mathrm{~cm}$ by $25 \mathrm{~cm}$. The sense planes for these wire chambers are atrung with the wires $2 t-30^{\circ}, 90^{\circ}$ and then $30^{\circ}$. The wire spacing is $0.2 \mathrm{~cm}$ in all sense planea, and the spacing between sense planes is $1.4 \mathrm{~cm}$. These chambers are composed of about 2,000 active wires (each) and are read out by the LBL wire chamber readout system.

The last counters that the particlea go though are the $A B$ counters. These are two rows of counters (the B's are clower to the target), with eight $A$ counters and ten $B$ counters. The counters are $0.7 \mathrm{~cm}$ thick and have an active height of $30.5 \mathrm{~cm}$. Three different widthe are ured $(33.1 \mathrm{~cm}, 19.1 \mathrm{~cm}$ and $9.5 \mathrm{~cm}$ ), which are staggered (as shown

\footnotetext{
'The origind wire chamber had been returned to its owners at the ead of the Fe rua and it we unavilable for these raso.
} 
in Figs. 4.1 and 4.2) to give 17 pousible overlaps. The staggering gives the finest possible granularity for the number of counters, which becomes finer towarda the high momentum end (the downstream end) of the array. Some of these counters were first used in 1979, and the pulse height resolution has degraded noticeably in the older counters. This was considered acceptable for this experiment since the time of flight information alone can give particle identiflcation for mont of the accepted momenta.

Downtream of the target, and not on the flight path of the pione, is an ion chamber uned to monitor the beam intonuity[1]. The output la paned to an Ortec 439 current intecretor and then to the CAMAC where it is the recorded by the computer.

The algnals from the various parts of the experiment were lead out of the experimental area to the electronics shack, were the tricger logic is made. The remainder of the description of the electronics is given in the next section.

The acceptance of the spectrometer for the Fe retup in given on a rapidity vs. $P_{\perp}$ plot in Fig. 4.3. The data shown are Monte Carlo data, with one track per event, weighted to reflect the particle emission probability[39, 40]. The figure contains both the $45^{\circ}$ and the $0^{\circ}$ acceptances. The lines marked " $A^{n}$ are the lower of the two momentum cuts employed for each of the two acceptances. The lines marked " $B$ " are the higher of the two momentum cuts employed. These cuts are explained in detail in Chapter 6, and the values will be repeated here. For the $45^{\circ}$ data, the cuts were $\left|\vec{p}_{\mathrm{cmal}}\right|>100 \mathrm{MeV} / \mathrm{c}$ and $\left|\vec{p}_{\mathrm{cm}}\right|>150 \mathrm{MeV} / \mathrm{c}$. For the $0^{\circ}$ data the cuts were $\left|\vec{p}_{\text {proj }}\right|>30 \mathrm{MeV} / \mathrm{c}$ and $\left|\vec{p}_{\text {proj }}\right|>100 \mathrm{MeV} / \mathrm{c}$. Table 4.4 gives the corresponding apectrometer characteristica.

\begin{tabular}{|cccc|}
\hline$\theta_{\text {lat }}$ & $\theta_{\mathrm{cm}}$ & $\left|\vec{p}_{\mathrm{cm}}\right|$, accepted & Solid Angle \\
\hline$\sim 0^{\circ}$ & $\left(0^{\circ}, 32^{\circ}\right)$ & $(100,400) \mathrm{MeV} / \mathrm{c}$ & $12 \mathrm{msr}$ \\
$\sim 45^{\circ}$ & $\left(91^{\circ}, 106^{\circ}\right)$ & $(100,600) \mathrm{MeV} / \mathrm{c}$ & $29 \mathrm{msr}$ \\
\hline
\end{tabular}

Table 4.4: Spectrometer characteristics for the Fe setups.

Table 4.5 gives the locations of the effective edges of the magnets, and the centers of the wire chambers. Using these positions, the field values from Table 4.2 and the effective edge approximation, one can reproduce the acceptance of the apectrometer. The coordinate system used is: the beam direction is $-\hat{y}, \hat{z}$ is vertical and $\hat{x}$ is perpendicular to the beam direction. The values are for the Ar/Nb setup, the values for the Fe set up 




Figure 4.3: Contour plot of the acceptance for the Fe retups, both $45^{\circ}$ and the $0^{\circ}$. The arow marks the beam rapidity, The lines marked " $A$ " are the lower of the two momentum cuts employed each acceptance, the lines marked "B" are the higher of the two cuts. 


\begin{tabular}{|c|ccc|}
\hline & \multicolumn{3}{|c|}{ location (cm) } \\
\hline \hline item & $x$ & $y$ & $z$ \\
\hline Beam direction & - & $-\hat{y}$ & - \\
Target pocition & - & 26.0 to 0.0 & - \\
C magnet effective edge & 41.0 & - & - \\
Wire Chamber 1 & 48.7 & -48.2 & 0.1 \\
Wiso Chamber 2 & 78.5 & -80.2 & -0.1 \\
Januu magnet effective edge & 126.6 & - & - \\
Jar'ue magnet effective edge & 204.8 & - & - \\
Wire Chamber 3 & 237.8 & -142.6 & -0.6 \\
Wire Chamber 4 & 316.9 & -142.4 & -0.2 \\
\hline \hline
\end{tabular}

Table 4.5: Locations of the principle components of the Janus spectrometer as used in 1986.

are similar. The effective edge approximation is discussed in detail in Chapter 5.

\subsection{Fast Electronicis and Computer Readout}

The trigger logic used in this experiment is given in Figs. 4.4 and 4.5. A few comments about the trigger logic diagram will be made here. The "Run Gate" was the output from a Jorway output register, and was used to allow the computer to enable and disable the triggers to start and stop the runs. The "Computer Free" was the inverse of the "Computer Busy" output from the trigger module that was set when the module was triggered, and remains set until the trigger was cleared by the computer. The "Fast Hold Off" (from "Event" to the "Master Gate") was used to disable the "Master Gate" until the "Computer Busy" can be aserted.

Once the event was triggered the data were acquired using standard CAMAC modules, read out by a program running on a Bi Ra MBD-11 connected to a computer. For the Fe data the computer was a PDP-11/45, and for the Ar/Nb data the computer was a VAX11/750. The PDP-11/45 based aystem could acquire about 200 events per spill (limited by the tape writing during the spill), whereas the VAX-11/750 based system could acquire 
Signals from Hardware

Signals to/from Electronics
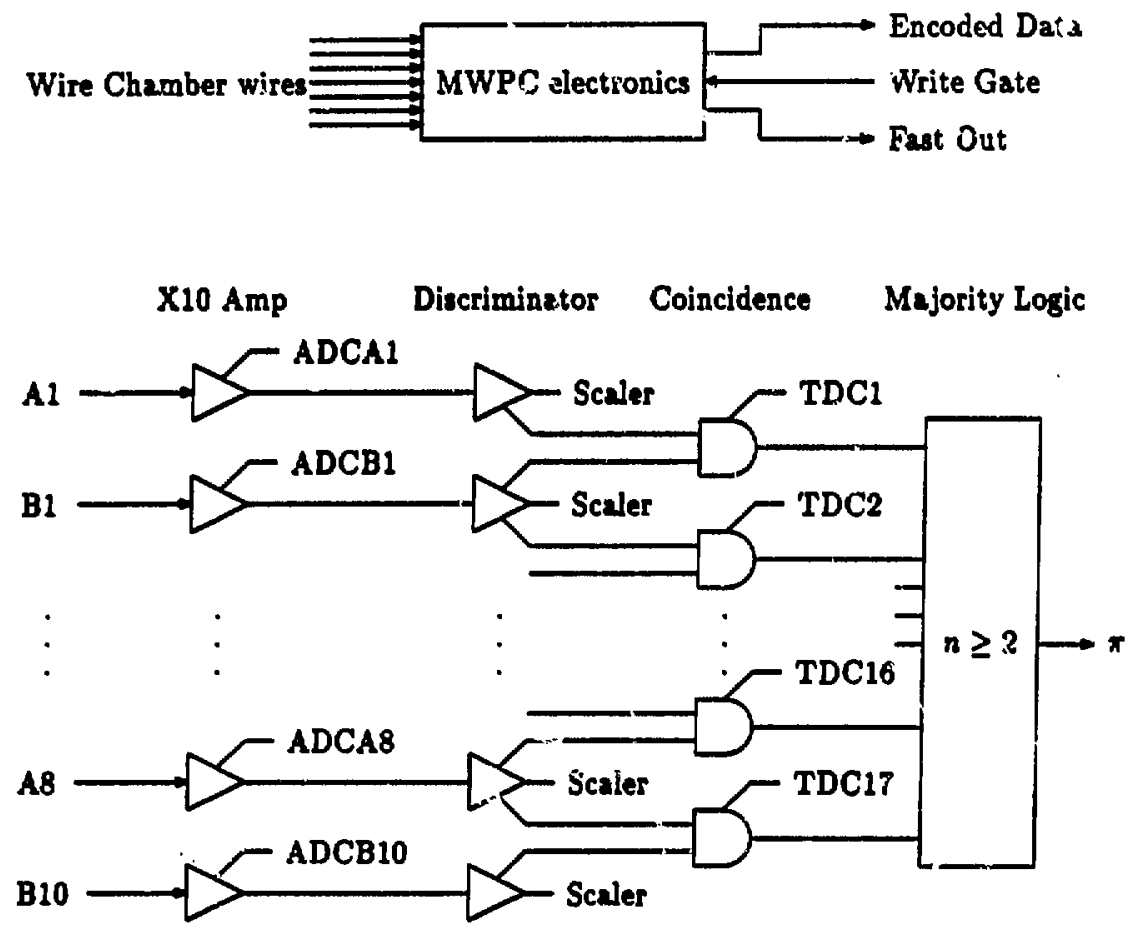

X10 Amp Rise-time Compensated

Coincidence

Discriminator

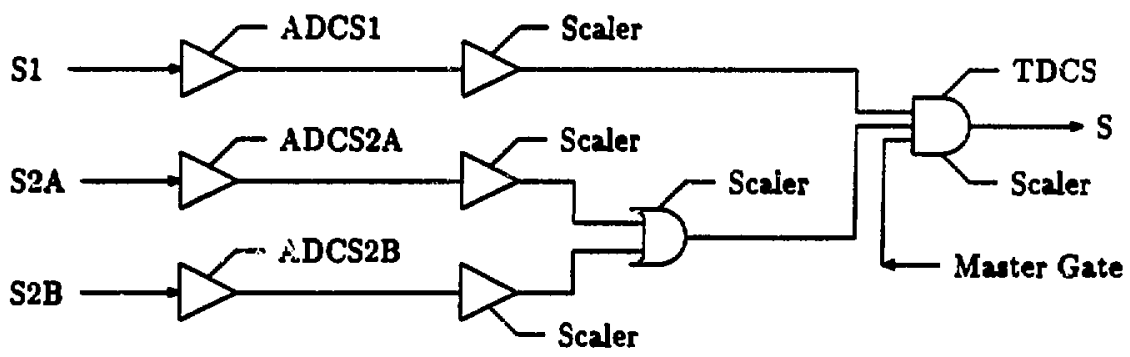

Figure 4.4: Trigger logic part 1. 
Signals from/to Electronics
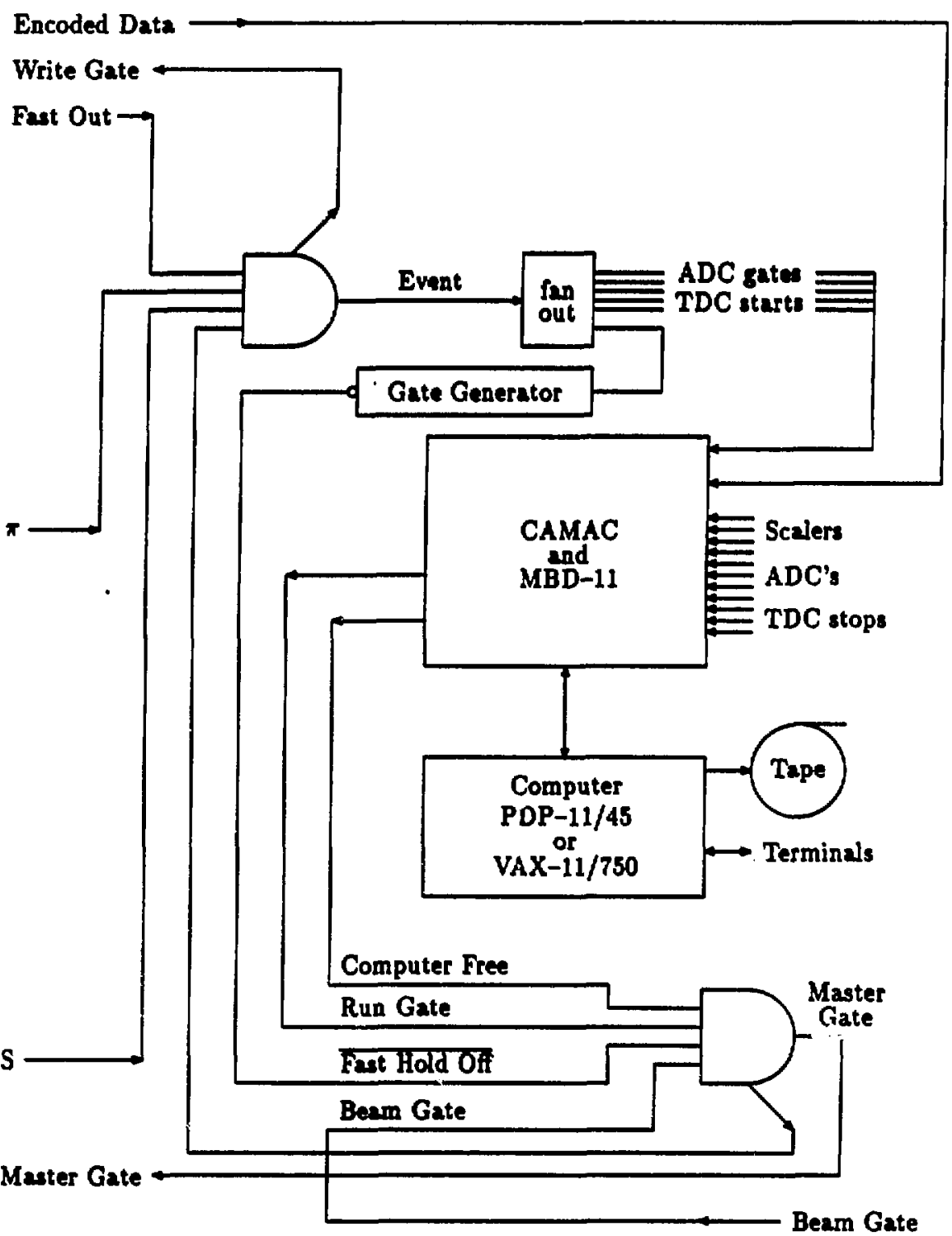

Figure 4.5: Trigger logic part 2. 
about 300 events per spill (limited by the event read-out speed). In addition, the VAX$11 / 750$ based system could do significantly more on-line analysis.

The slow logic, used to synchronize the experiment and the computer to the accelerator beam spills, is shown in Fig. 4.6. When the VAX-11/750 based system was in use, the "Start of apill" and "End of spill" events were used to disable the 11/750's analysis of event data during the spill so the $11 / 750$ could tranafor data into its memory at the largeat rate posaible.

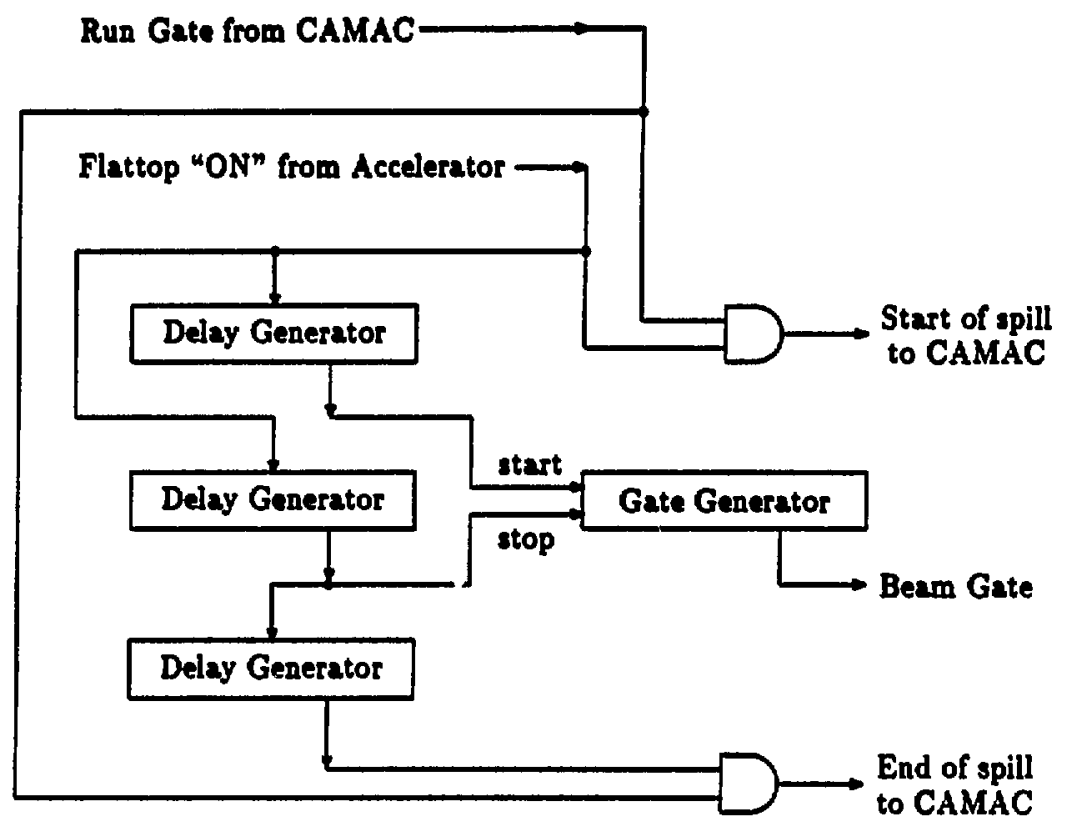

Figure 4.6: Slow Logic.

The data acquisition programs running in the MBD-11 and the PDP-11/45 (or VAX11/750) were the $Q$ aystem[41]. These programe were written (and maintained) by the MP-1 group at Los Namos and the Real Time Systems group at Lawrence Berkeley Laboratory. The author wishes to thank the group for the use of their programs and their efforts in correcting the few buge that showed up during the experimental runs.

\footnotetext{
'In 1988, the MP-1 group became Group MP-6, LAMPF, Los Alamos National Laboratory.
} 


\section{Chapter 5}

\section{Track finding and Particle Identification}

\subsection{Outline of the Problem}

Track finding and particle identification in the two pion experiment are broadly divided into three taks. First, there is finding the hits in the wire chambers given the raw data (the wire numbers of the struck wires). Second, there is finding the tracks given the hits in the wire chambers. Third, there is particle identification, and momentum determination for each track found. The programs (or subroutines) to accomplinh the earlier tasks are faced with the simultañeous requirements of being fust enough to look at almost every event in the experiment, but also complete enough not to introduce statistical biases that will complicate the later analysis. This must be done in an experiment that requires two tracks in the spectrometer for a good event. In addition, the region of most interest, that of low momentum differences, is where the two tracks are physically close together and, therefore, easier to confuse. A further complication for the Fe data set was that the track finding was originally done on a PDP-11/45 and the program had to fit into the addreas apace atiowed by the 16 bit addreasing.

The size of the calculation task is illustrated with the track finder. In the ideal case of just two real tracks, there are four posaible out-going (from the Janus magnet) tracks defined by the two bits in each of the two rear wire chambers. Similarly, there are four 
possible in-going (to the Janus magnet) tracks defined by the two hits in each of the two front wire chambers. Therefore, the eight hits in the four wire chambers define sixteen possible candidates for the first track (once the first track is found, there is only one choice for the second track in this case). Although some of the candidates for the track can be eliminated early in the calculation, ance the candidate in obviously bad, the presence of stray particles atriking the wire chambers increaser the number of candidaten to conaider and, to an order of magaitude, the aumber of candidaten that have to be considered for the firnt track is an order of magnitude larger than the number of events.

\subsection{Hit Finding}

The hit finding routines work a follows: The wire chambers were positioned so that the tracks were approximately perpendicular to the wire chambers. It is then possible to ignore the dimension perpendicular to the wire chamber, which is approximately parallel to the particle's path, approximating the three separated sense planes of the wire chamber by three sense planes which are superimponed. If a particle goes though the wire chamber and fires all three planes, the hit will be located by the three fired wires that pass through a single point. In general, due to the finite resolution of the wires and non-normal track, the three wires will not define a single point, but will define a small triangle. The program must calculate the size of this triangle and use the size to decide if a triplet of wires forms a hit or not. The method chosen must be simple enough to calculate for every possible triplet of wires in every wire chamber for every event.

To derive the method used here, it is first necessary to define the symbols used:

Let $N_{i}$ be the number of the wire of interest for plane $i$.

$C_{i}$ be the number of the wire passing through the center of the wire chamber for plane $i$.

$w_{i}$ be the separation of the wires for plane $i$.

$\bar{n}_{i}$ be the unit normal to the wire, in the direction of increasing $N$, for plane $i$.

In Figure 5.1 these definitions are illustrated in a wire chamber for a single hit within the wire chamber. In this experiment $N_{i}$ is chosen, for all wire chambers, to increase going 


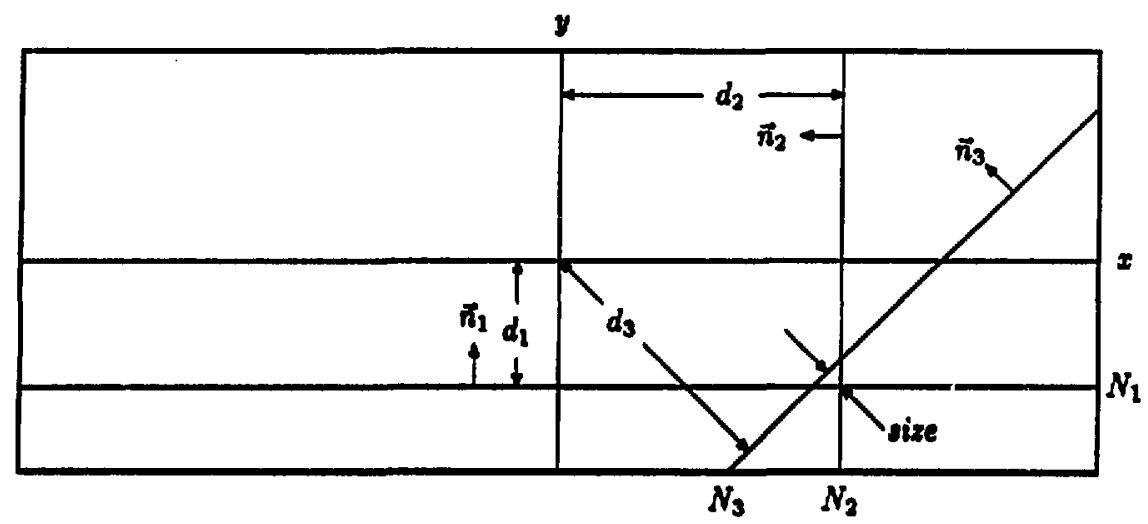

Figure 5.1: A triangie in a wire chamber.

to the left for non-horizontal wires, or up for horizontal wires, a drawn. The (signed) distance from the center of the wire chamber (which is taken as the origin) to the wire is

$$
d_{i}=w_{i}\left(N_{i}-C_{i}\right)
$$

so the wire is given by $\vec{x}$ such that

$$
\vec{x} \cdot \vec{n}_{i}=v_{i}\left(N_{i}-C_{i}\right)
$$

A "cross" is defined as the intereection of two wires. The location of a cross is a simultaneous solution to two of Eq. 3.2 for the two different planes of wires. In component notation this is $(x, y)$ such that

$$
\begin{aligned}
& x n_{x 1}+y n_{y 1}=w_{1}\left(N_{1}-C_{1}\right) \\
& x n_{x 2}+y n_{y 2}=w_{2}\left(N_{2}-C_{2}\right)
\end{aligned}
$$

Assuming that

$$
\left|\begin{array}{ll}
n_{x 1} & n_{y 1} \\
n_{s 2} & n_{y 2}
\end{array}\right| \neq 0
$$

otherwive the wires are parallel, and none of the wire chambers in this experiment have different planea with parallel wires, the matrix can then be inverted and $x$ and $y$ are given 
by

$$
\left(\begin{array}{l}
x \\
y
\end{array}\right)=\frac{1}{\left|\begin{array}{ll}
n_{x 1} & n_{y 1} \\
n_{x 2} & n_{y 2}
\end{array}\right|}\left(\begin{array}{cc}
n_{y 2} & -n_{y 1} \\
-n_{x 2} & n_{x 1}
\end{array}\right)\left(\begin{array}{l}
w_{1}\left(N_{1}-C_{1}\right) \\
w_{2}\left(N_{2}-C_{2}\right)
\end{array}\right) .
$$

Putting this into Eq. 3.2 for the third plane, the wire number for the third plane is given by

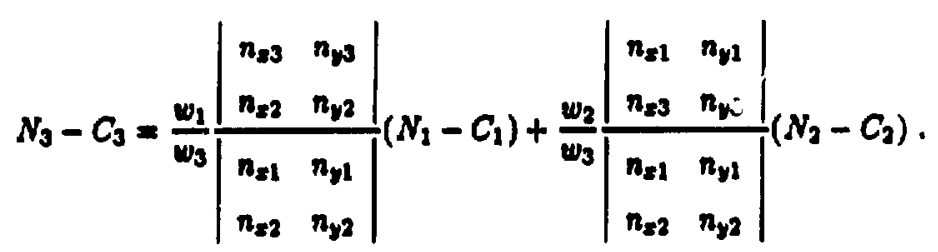

Hence, for a triangle of zero size

$$
v_{1}\left|\begin{array}{ll}
n_{x 3} & n_{y 3} \\
n_{x 2} & n_{y 2}
\end{array}\right|\left(N_{1}-C_{1}\right)+w_{2}\left|\begin{array}{ll}
n_{x 1} & n_{y 2} \\
n_{x 3} & n_{y 3}
\end{array}\right|\left(N_{2}-C_{2}\right)+w_{3}\left|\begin{array}{ll}
n_{x 2} & n_{y 2} \\
n_{x 1} & n_{y 2}
\end{array}\right|\left(N_{3}-C_{3}\right)=0 .
$$

The above equation is included on account of its aymmetry and because from it the third wire through a hit can be found if the other two wires are known. The equation used is that which gives the difference between the real $N_{3}$ wire and that predicted by the cross of $N_{1}$ and $N_{2}$

$$
\Delta N_{3}=\frac{w_{1}}{w_{3}} \frac{\left|\begin{array}{ll}
n_{x 3} & n_{y 3} \\
n_{x 2} & n_{y 2}
\end{array}\right|}{\left|\begin{array}{ll}
n_{x 1} & n_{y 1} \\
n_{x 2} & n_{y 2}
\end{array}\right|}\left(N_{1}-C_{1}\right)+\frac{w_{2}}{w_{3}} \frac{\left|\begin{array}{ll}
n_{x 1} & n_{y 1} \\
n_{x 3} & n_{y 3}
\end{array}\right|}{\left|\begin{array}{ll}
n_{x 1} & n_{y 1} \\
n_{x 2} & n_{y 2}
\end{array}\right|}\left(N_{2}-C_{2}\right)-\left(N_{3}-C_{3}\right) .
$$

There is a small computational complication. The resolution of the wire chambers is half integral (in terms of wire spacings). Since the computers store integers more efficiently than fractional numbers, it is deairable to use twice the wire number instead of the wire number, since twice the wire number is integral. For this reason the equation actually 
used is

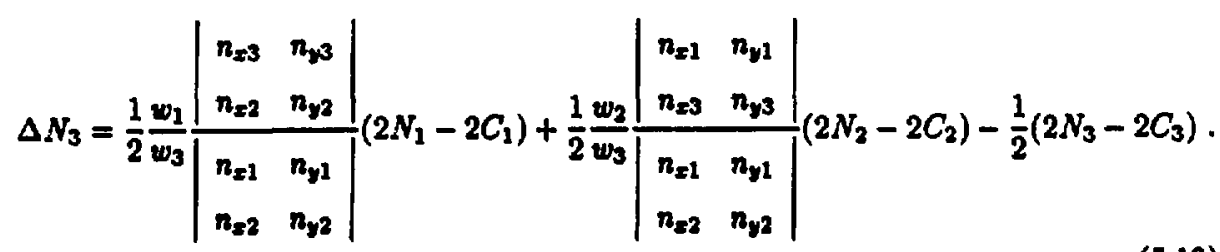

The coefficients of $\left(2 N_{i}-2 C_{i}\right)$ are celculated once for this experiment and entered into the codes. The $2 C_{i}$ are entered into the codea, 10 that the calculation of a triangle size requires 1 addition, 4 eubtractlone and 3 multiplications, and can be done for each triplet of wires. A further anving could have been realied by clearing the parenthesis and adding all the conatants together. However, it was felt that the increased chance for error when varying the center wire numbers offeet the possible gain and this was not done.

The hit finding process starts with each triplet of wires being checked against Eq. 5.10 and if the aize is leas than the maximum size ( 5 wires) the triplet is considered a triangle and a poasible hit.

Experience has shown that hits cannot be allowed to share wires. That is, a tingle wire cannot be allowed to be part of two different hits. If this is allowed, the distribution of the second hit relative to the first shows clear indications that the number of hite sharing wires is greater than one would expect for a random distribution. So the hit finding proceeds as follows: All triangles smaller than the maximum size are stored as hit candidates. If too many candidates are found the event is rejected to avoid biases. The triangles are ordered by size (smaller first) and each triangle in checked for shared wires with previously found hits. If there are none, the triangle is considered good and is added to the list of found hits and its wires ancociated with the hit. If there are shared wires then the triangle is rejected. The smalleat triangle is, of course, always considered a good hit.

This is best illustrated as in Fig. 5.2, where a typical three bit event is shown. The wire angles are thone of the first and second wire chambers, however a similar picture could be drawn for the third and fourth wire chambers. There are potentially five triangles, labelled 1-5, and 3 shared wires, labelled $N_{1}, N_{2}$, and $N_{3}$. The four reasonably small hits are labelled 1-4. The requirement of small triangle size eliminates the triangle near 5 as a hit, but the assignment of the hits based on triangle size alone is ambiguous for 


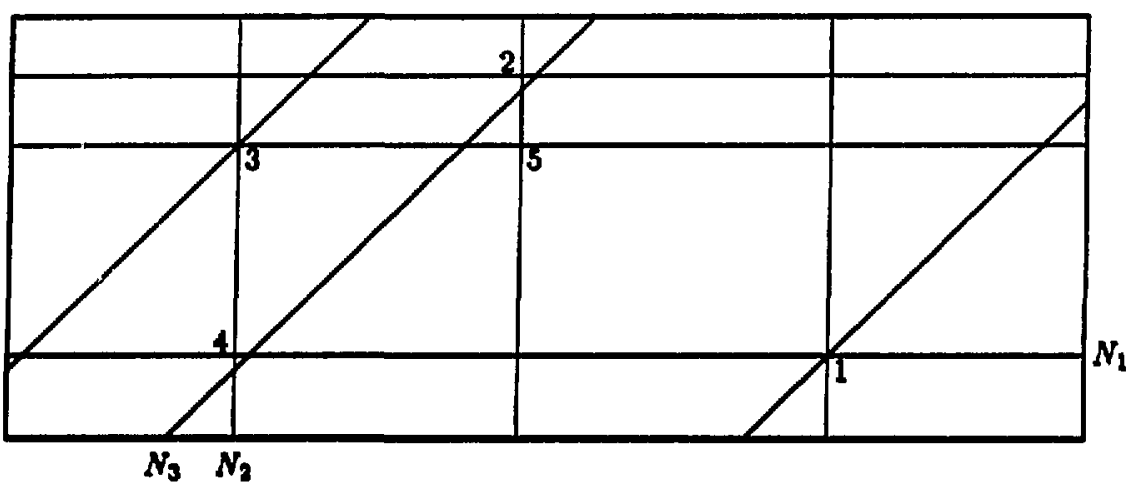

Figure 5.2: Thies hits in a wire chamber ahowing pousible shared wires, $N_{1}, N_{2}$, and $N_{3}$.

the triangles near 2 and 4. However, following the preference for small triangles, then the wires for the hits near 1 and 3 should be removed, so that it is not possible for these wires to be shared with other hits. It is then clear that 2 is the remeining hit.

It has been anumed that the wire chambers are $100 \%$ efficient. In reality, the wire chambers were measured to be about 95\% - 99\% efficient. Taking 95\% as an example, a two-track event with 24 struck wires and efficiency of $95 \%$ per wire will give an event efficiency of $0.95^{24}$, or $54 \%$. Clearly, it cannot be required that all hits be perfect triangles, but miscing wires must be allowed. ${ }^{1}$ So the wires that are not associated with good triangles are checked to ace if they crow within the chamber boundaries, if so they are called a hit, and the position is recorded. At this stage, hits formed from crosses are alowed to share wires with other crosses.

A side effect of the procedure is that the hits are stored in the arrays as smalleat triangles first, then larger triangles, and then croases. When track finding is done the hit arrays are searched in this order, and the track finding is biased towards smaller triangles.

At this point the number of hits in each chamber is counted. If there are not at least a many hits in each chamber a tracks sought, no further proceasing of the event is done.

\footnotetext{
'The trect-fider's eflciency for wing the wirw is mevented to be abont 85\%. So from the treckfader's point of viow it is emential 10 sllow miming wirm. The diference between the track-finder's eficieacy and the wire-chamber's exiciency in due to the shared-wire removel in the hit finder and the treck finder.
} 


\subsection{Effective Edge Track Finding}

Before starting with the effective edge work, recall that the Janus spectrometer (see Figs. 4.1 and 4.2) consiats of two dipole magnets, the $C$ magnet near the target, and the Janus magnet. Between the $\mathrm{C}$ magnet and the Janus magnet are two wire chanbers, and after the Janus magnet are two more wire chambers. The deflection in Janus gives the particles' momentum.

Once the bits in the wire chambers have been found, the locations of the hits are pased to the track fnding routine. To allow the track finding to proceed at a reusonable pace, ${ }^{2}$ the track findlug is done in the eficetive edge approximation, with vertical focusaing. In this approximation, the feld is asumed to be sero outside the magnet and at (conatant) full feld invide the magnet. The boundarion for "inside" and "outside" can be chosen to either give the beat approximation te the real field in the eenne of the real tracks are well fit by the effective edge tracks, or that the deflection given by the effective edge field (when set to the central value of the real field) is a good approximation to the deflection in the real field. Studies of Monte Carlo tracks using the field map show that for Janus the two choices are nearly the same. In the $\mathrm{C}$ magnet the effective edge porition was choeen to give the best agreement for the target traceback position and initial angles of the momentum vector.

The motion of the particles is either that of a straight line, in the field free regions, or that of a section from a spiral (with the radius determined by the Lorentz force law), in the full field regions. Viewed parallel to the field, the track follows an arc in the full field regions. The fields in the $\mathrm{C}$ magnet and the Janus magnet are both vertical, so in the plan view the tracks follow arca inside the full field regions of the $\mathrm{C}$ and the Janus magnet. One can easily show ${ }^{3}$ that at the boundaries the track is continuous, the derivatives in the horizontal directions are continuous and the vertical derivative (臬) has a jump discontinuity given by

$$
\Delta\left(\frac{d z}{d 0}\right)=\frac{\tan t_{0}}{r}
$$

where $s$ is the path length, $z$ is the vertical coordinate ( $z_{0}$ is the height of the path above

\footnotetext{
${ }^{2}$ The hit and track finding progran wa originally written to rum on a PDP-11/45, and attained a speed of about 10 events per second on the PDP-11/4s.

'Sine, for example, [12].
} 
the magnet's mid-plane), $r$ is the radius of curvature, and $\theta$ is the angle between the particle's path and the normal to the field boundary.

In the track finding program the effective edge motion is used as follows. The first step is to take all the hits in the firat two wire chambers and generate all the in-going (to Janus) vectors. If the $\mathrm{C}$ magnet is turned off, the in-going vector is traced back to the target plane and the position is checked. The next stop is to take all tho hits in the last two wire chambers and generate all the out-going (from Januu) vectors. Then a simple seometric check is made to $\mathrm{me}$ if a circle (In the plan viow) can be made in the Janus magnat uning the vectorn.

The offective edse approximation requires that the circle (in the full field region) be tangent to the in-going and out-going vectors at the point the vectors intersect the effective edges of the magnet. Recall that for any point on a circle, the line from the center of the circle to a point on the circle is perpendicular to the tangent at that point.

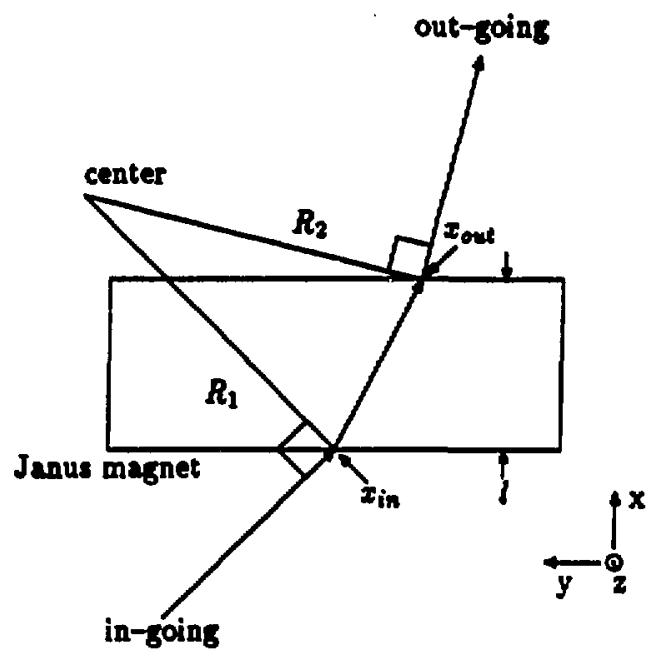

Figure 5.3: The Janus magh : with the eet up for the $\Delta r$ calculation.

The set up is shown in Fig. 5.3, the points $x_{\text {in }}$ and $x_{\text {ont }}$ are the intersection of the in-going and out-going vectors with the effective edges. The lines $R_{1}$ and $R_{2}$ are perpendicular to the in-going and out-going vectors at $x_{i n}$ and $x_{\text {out }}$. If the two vectors are 
from a good track, then the center of the circle is the intersection of $R_{1}$ and $R_{2}$. Then $R_{1}$ and $R_{2}$ are two radii of the circle that the path defines, and the fractional difference, $\Delta r=\left(R_{1} / R_{2}\right)-1$, will be small. It is an eany exercise to calculate $R_{1}$ and $R_{2}$ from the length of the side of the triangle from $x_{i n}$ to $x_{\text {out }}$, the angle between $R_{1}$ and $R_{2}$, and the sine law. The choice of form for $\Delta r$ was made to make the calculation insensitive to round-off errors. However, if both $R_{1}$ and $R_{2}$ are large, then the fractional error could be small, whereas the difference could be large. Therefore 2 cut is imposed 10 that $R_{1}$ and $R_{2}$ are not more than soveral times a large $a$ the radius of the least curved track. The tracks at this point are ordored by $\Delta r$ so that the "beat" tracks are checked firat.

Since two tracks are desired, all the steps that follow are done for both tracke.

- First, the momentum ${ }^{4}$ is calculated from deflection in the Janus magnet. One can easily show ${ }^{3}$ that

$$
p=\frac{q}{c\left|\sin \theta_{\text {in }}-\sin \theta_{\text {out }}\right|}
$$

where $l$ is the effective edge field length, $\theta$ is the angle between the particle's path and the normal to the field boundary (in for entering the full field region and out for leaving), $B_{0}$ is the field value at the center of the magnet, $c$ is the speed of light, and $q$ is the particle's charge." The procedure adopted for calculating $/$ wa to calculate $\Delta$ ain $\theta$ for a sample of Monte Carlo tracks (made using the full field map). Then uning the known momentum of the track, and the field $\left(B_{0}\right), l$ is calculated. The momentum of the real tracks is then found from l, $B_{0}$ and the $\Delta \sin$ of the track.

Once the a omontum is known (Eq. 5.12) then the vertical motion can be calculated using Eq. 5.11. The motion can be easily expressed in matrix notation where the vector of interest is

$$
\left(\begin{array}{l}
2 \\
y
\end{array}\right) \text {. }
$$

Then motion from one point to another an arc length $S$ apart in a constant field region

\footnotetext{
'The monentun cakculated bere is ouly und in the efective edse track finding. Soe Soction 3.6 for the decription of how the find momenture is found.

See, for excuple, [1].

This is the only location where, will be und for charge, cleswhere it is the diference in the momentum of the piona.
} 
is just

$$
\left(\begin{array}{c}
z \\
y
\end{array}\right)_{\text {out }}=\left(\begin{array}{ll}
1 & s \\
0 & 1
\end{array}\right)\left(\begin{array}{c}
z \\
z y
\end{array}\right)_{\text {in }},
$$

and the deflection at the field boundary is

$$
\left(\begin{array}{l}
z \\
d
\end{array}\right)_{\text {ont }}=\left(\begin{array}{cc}
1 & 0 \\
-j & 1
\end{array}\right)\left(\begin{array}{c}
x \\
y
\end{array}\right)_{\text {in }},
$$

where $\}=m_{r} t_{20} a$ in Eq. 5.11 .

The motion from the first to the recond wire chamber given the initial $y_{0}$. Then the remainder of the motion can be found by applying Eq. 5.14 to move the particle from one location to the next, and applying Eq. 5.15 to move the particle acrost the field bounduries.

In the Janus magnet a further aimplification is made; because the arc length $(S)$ is hard to calculate it is deaired to replace it with one of the coordinates, $x$. In the field free regions this change of variables is trivial, and the transfer matrix becomes

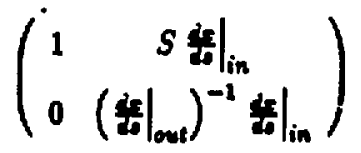

and in the field free regioss $\frac{d x}{d x}$ is conotant. All the need be done is to replace $S$ by $X$, the change in $x$, to recover the original form of the transfer matrices.

In the full field regions cyout is not necessarily equal to to lin and is certainly not constant. However, to the accuracy of the other approximations made in the derivation, this is assumed to be true, and the full field regions are calculated in the same fashion as the field free regions. In the Janus magnet the tracks never make an angle of more than $\sim 43^{\circ}$ with the $x$ axis and generally less, 20 do varies around its maximum value of 1 . In the $\mathrm{C}$ magnet, the tracks are handled differently, as will be described below.

In a similar calculation, the deflection matrix beromes

$$
\left(\begin{array}{cc}
1 & 0 \\
\left(\left.x\right|_{\text {out }}\right)^{-1}(-f) & \left.\left(\left.x\right|_{\text {out }}\right)^{-1} \mathrm{xz}\right|_{i=0}
\end{array}\right) \text {. }
$$

Again, to the accuracy of the other approximations made in the derivation, we asesme

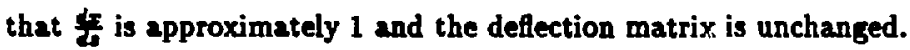


With these approximations, the transfer matrices and the deflection matrices do not depend on the path length and are easy to calculate. Using the appropriate transfer matrix from the second effective edge to the third or fourth wire chamber, the height of the track can be predicted in the third and fourth chambers. A cut is made requiring that the hits used be close to the predicted height. ${ }^{7}$ At this point the track finding in the Janus magaet is complete anc there is a good chance that the track found is a real one. Therefore, the omphals in the routines shifts from fast computing times to accuracy.

Next, the track from chamber three to chamber four is extended as a straight line to the AB array and the counters that the track atruck are recorded. Tracks that mias the array are, of course, rejected.

If the $\mathrm{C}$ magnet is on, then the target traceback is done here. This is done to the full accuracy of the effective edge approximation because the tracks start out parallel to the beam line and the approximation ured in the Janus magnat fails. The track is projected from the second chamber to the first chamber, and then to the effective edge of the $C$ magnet. The radiun of the tr sck in the $\mathrm{C}$ magnet is computed from the Lorentz force law, and the new components of the velocity vector are computed from Eq. 5.11. The track then follows a spiral path back to the plane of the target. "The length of the spiral is computed, and then the position at the target plane. A cut is made requiring that the initial porition of the track be near the beam spot.

The tracks are then checked to see that the second track does not shase wires with the first. Recall that, although triangles cannot share wires with anything else, crosses were allowed to share wires with other crosses. So at this level, a cut is imposed so that crosses are allowed to share wires in the two tracks only if all other hits in the pair of tracks are triangles. This gives the distribution of hits closest to flat. If the second track passes this cut, the pair of tracks is considered to be a good event and the data are written to the output file for further procensing.

The accuracy of the effective edge approximation has two aspects. The first is the efficiency for finding single tracks, and the second is the efficiency for finding the two tracks correctly. When calculating the correlation function using the event-mixing technique, the single track efficiencies cancel out (see Section 6.2) and need not be measured. However, a

\footnotetext{
${ }^{7}$ These cuta are called $\Delta Z_{3}$ and $\Delta Z_{t,}$ respectively.
} 
good efficiency is desired to allow the largest data sample possible. The major concern is that two track efficiency depends in a predictable way on the event geometry. The track finding in single track and double track events were studied using Monte Carlo data using the full field map (in both magnets) with simulated multiple scattering and energy loss (with Lonidiu straggling). The Monte Carlo data were presented to the track finder with one track per event to check the single track case, and with two tracks per event to check the two track case. For the single track data, the results are given in Table 5.1. The crordinates used are: $\dot{z}$ is vertical, and $\dot{x}$ is horizontal, perpendicular to the beam. The quantities $\Delta Z_{3}$ and $\Delta Z_{4}$ are the differences between the predicted (based on the effective edge approximation) and the actual heights in the third and fourth wire chambers.

\begin{tabular}{|c|l|}
\hline Quantity & RMS error \\
\hline \hline Target traceback $x$ & $1.0 \mathrm{~cm}$ \\
Target traceback $z$ & $1.0 \mathrm{~cm}$ \\
$\Delta r$ & $0.79 \%$ \\
$\Delta Z_{3}$ & $0.69 \mathrm{~cm}$ \\
$\Delta Z_{4}$ & $1.0 \mathrm{~cm}$ \\
$|\vec{P}|$ & $3.4 \mathrm{MeV} / \mathrm{c}$ \\
\hline
\end{tabular}

Table 5.1: Effective edge parameters for one Monte Carlo track per event.

When two tracks are present in sach event, the possibility for misidentifying the hits exists. Therefore the histograms of the parameters include not only the effective edge errors as given in Table 5.1, but also random matches. These histograms are given in Fig. 5.4, 5.5, and 5.6. It can be seen that the effective edge approximation allows accurate track reconstruction.

With the Monte Carlo dat2, ihe tracks output by the track finder can be compared with the input. There exist three possibilities for an event: First, the event can be found correctly. Second, the event can be missed altogether, because not enough tracks can be found. Third, the event can be returned with the tracks confused, that is, with some hits from each input track assigned to one output track. The histograms for each of these classes for the first wire chamber are presented in Fig. 5.7. The figure shows the fraction of the input data in each class as a function of the distance between the two hits in this 


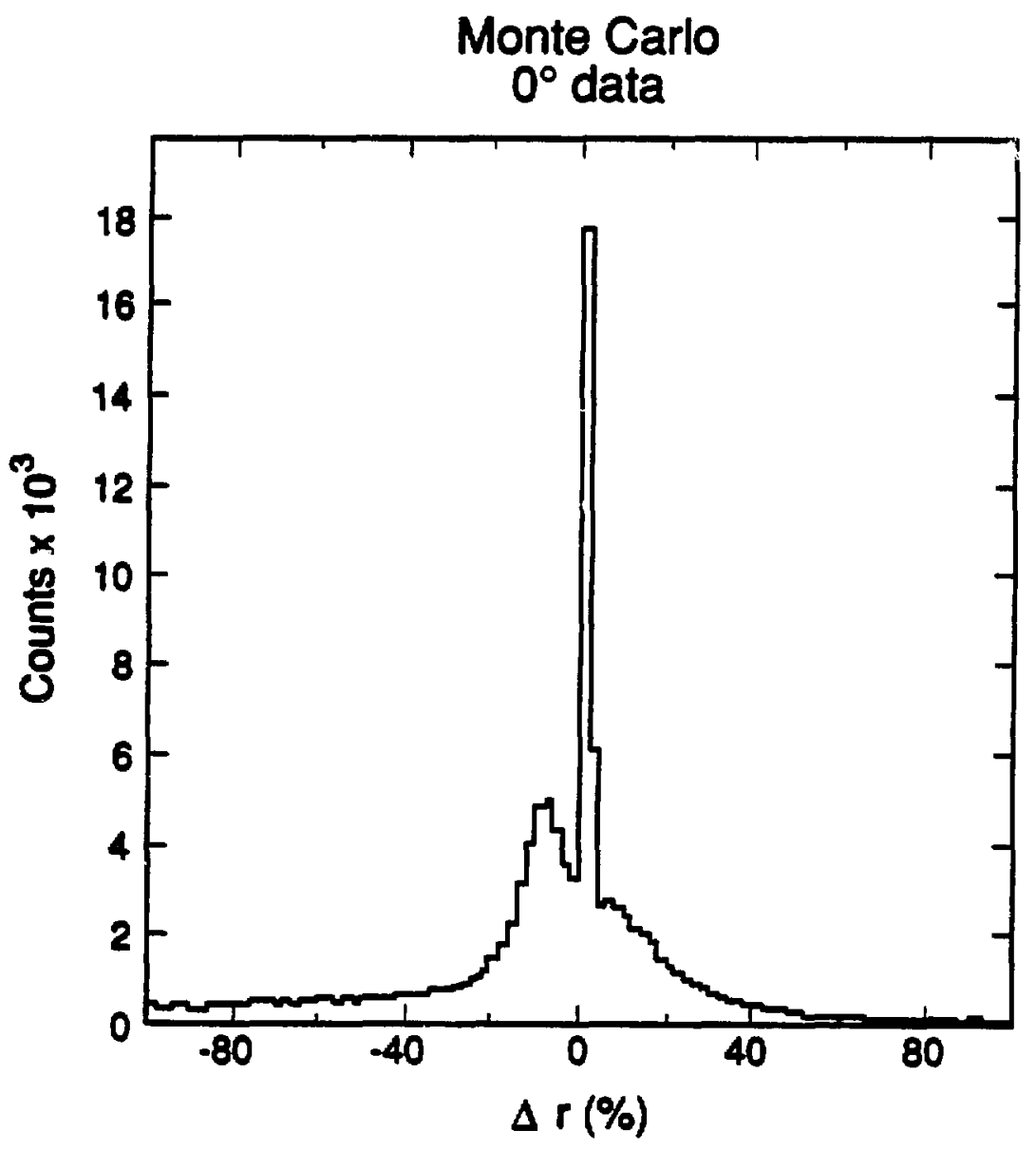

Figure 3.4: $\Delta r$ with two trackt per eveat. 


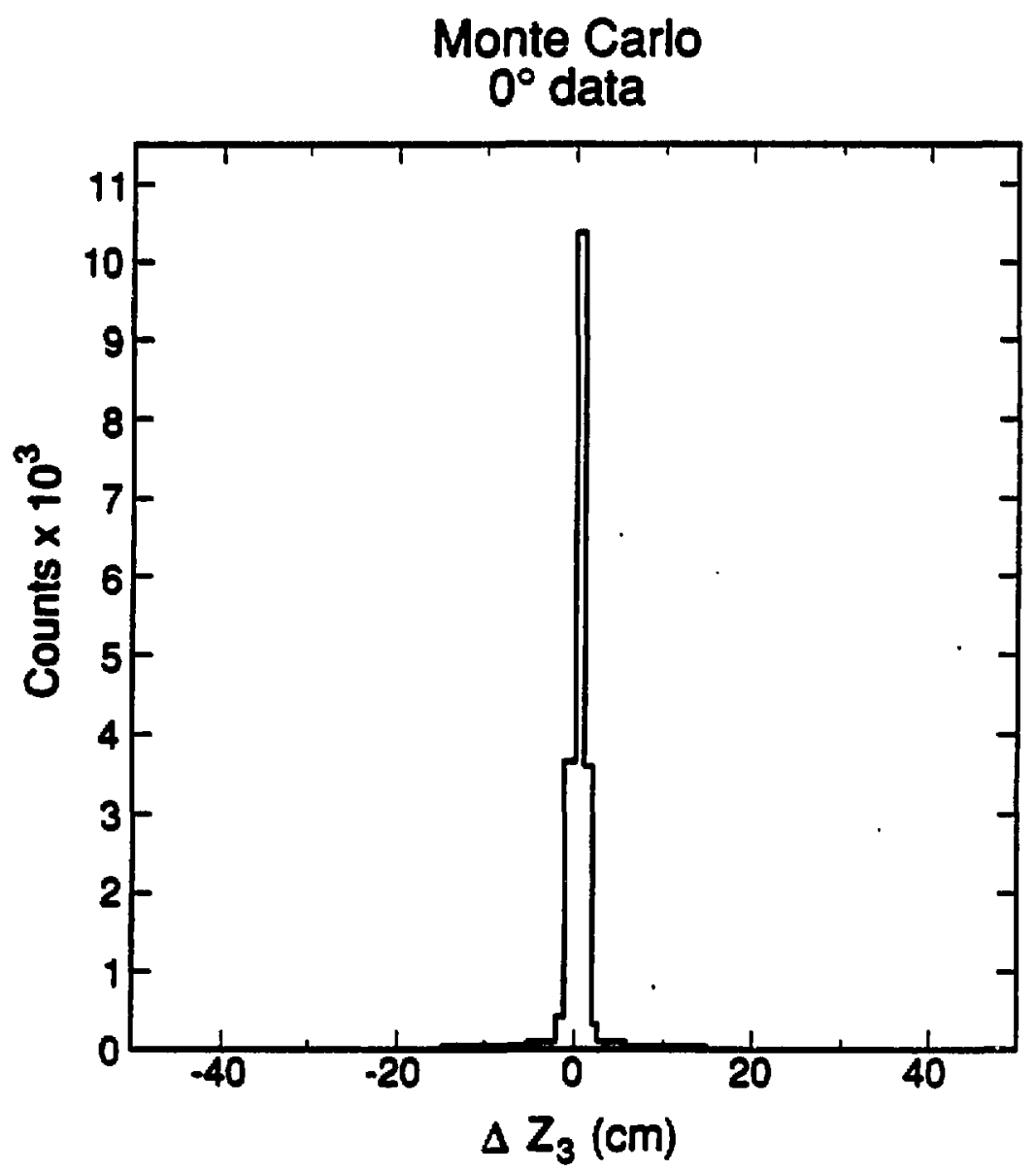

Figure 5.3: $\Delta Z_{3}$ with two tracks per event. 


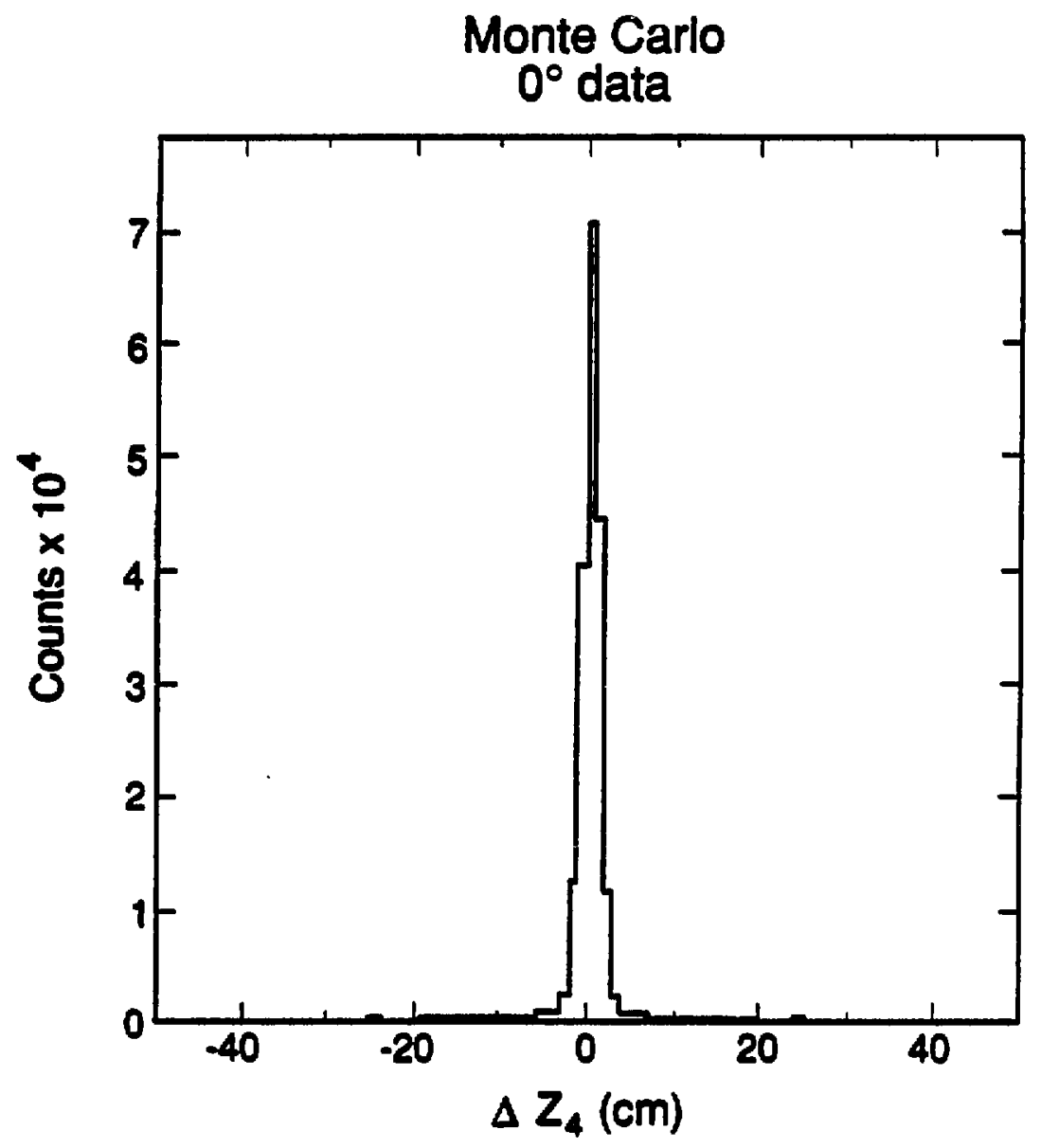

Figare 5.6: $\Delta Z_{4}$ with two trecks per event. 


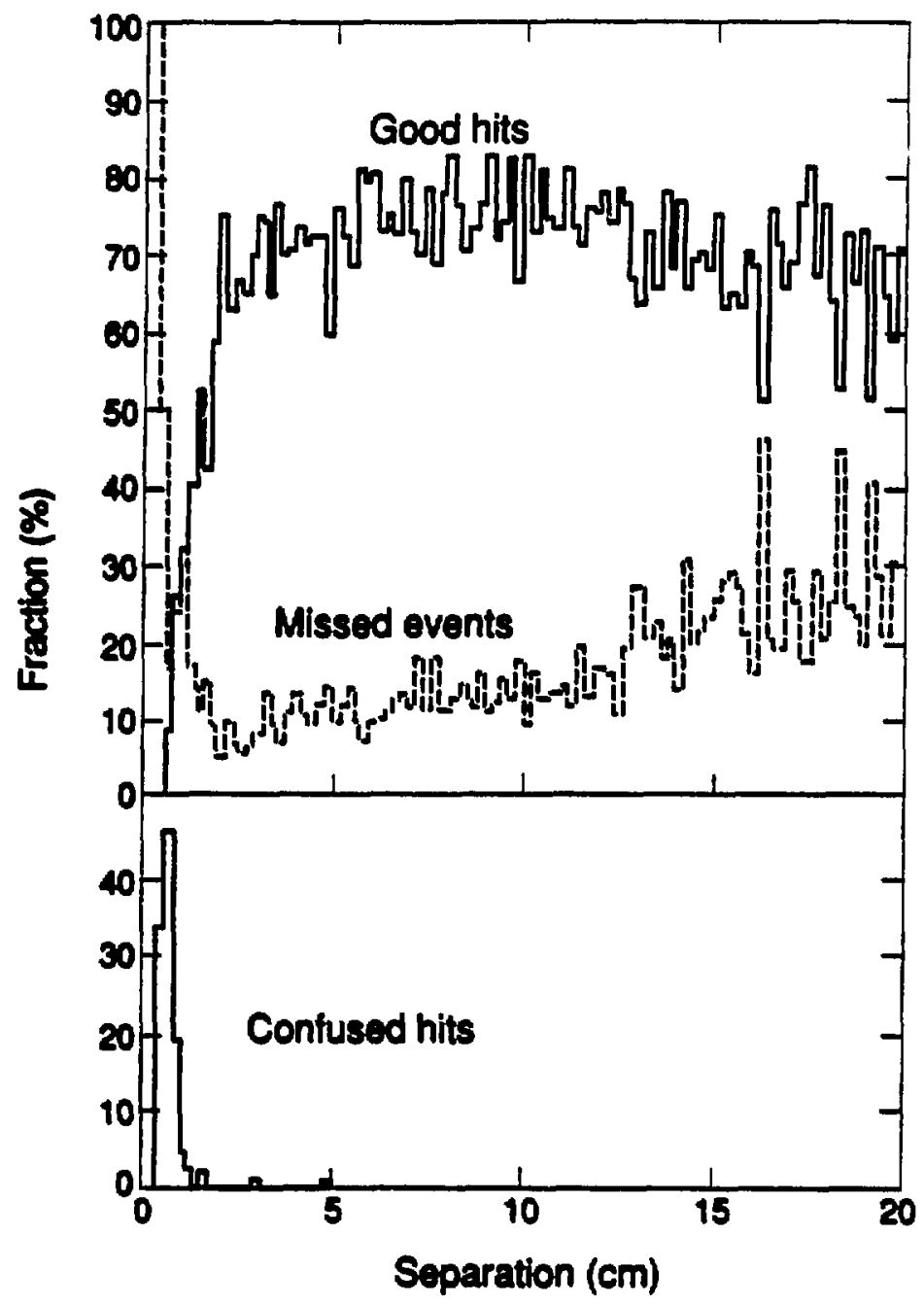

Figure 5.7: Track finding as a function of the hit separation in wire chamber one for Monte Carlo data. 
wire chamber. The classes shown are: good hits - where the two tracks in the event were found correctly, missed events - where the two tracks in the event were not found, and confused hits - where the hits in this wire chamber were not found correctly (although two tracks were still found in the event). The histograms for the other wire chambers are similar, although the fraction of events with low separations is smaller for the other wire chambers since they are farther from the target. The feature to notice is that for separation of greater than $2 \mathrm{~cm}$ the fraction of confused hits is easentially zero, and the fraction of correctly found ovents is approximately constant. This is discussed further in the next rection (Section 5.4).

At this point track finding is considered complete and the event information is written to tape to allow further proceuing. About $10 \%$ of the triggers ourvive the firat level prograns to be written to tape. Before discussing the further proceasing, the two track efficiency problems and their intuence in the data analysis will be discussed.

\subsection{Two Track Efficiency}

The track-finding routines used introduce some two track efficiency effects. Correcting for them requires understanding how they are introduced in the real data, so that they can be simulated in the background data.

First, there is the hardware trigser requirement. Since the trigsering requirement it - that two independent $A B$ pairs fire, events where both tracks strike the same $A B$ pair will not fire the hardware trigger. Although it is possible that two AB pars will fire for some reason when only a single one was struck by the two particles (for example, from a $\delta$-ray striking a nearby counter), it is far more likely that a single pion event will cause this trigger than a two pion event, simply because of the large number of single pion events in the spectrometer. Therefore, a cut is made that requires that the two tracks trace to different $A B$ pairs for the event to be accepted.

Second, the requirement that the triangles do not share wires means that the probability for detection of two hits that do share wires is reduced. This is because if a wire is missing from either of the two triangles, the remaining hit (call it the first hit) will form a triangle and the shared wire will be assigned to it, then the second hit will have only one wire passing though it and it is not possible to form a crose to recover the second 
hit. Attempts to relax this requirement have always produced data where the number of tracks sharing wires is larger than one would expect for a random hit distribution. Rather than attempt to calculate the probability of missing hits in this fashion, which would depend on the plane efficiencies, a cut is made requiring that the two hits be such that, if all planes fired, the separation of the centroids of the clumps of fired wires in all planes is greater than or equal to 3.5 wires. The choice of 3.5 wires is determined by the requirement that there must be an unfired wire between two clumps of fired wirea for the fired wires to be realved as separate clumps of wires. Allowing one of the clumps to be two wires wide, this implies that the minimum distance between the centroids for the clumpe to be resolved is 2.5 wires. Allowing for the wire chamber resolution of $\pm 1 / 2$ wire for each of the hits implies that the minimum separation between hits to guarantee that the clumpe are resolved is 3.5 wires.

Third, the effective edge track reconstructlon confuses hits in the tracks when the hits are separated by less than $2 \mathrm{~cm}$. The track identification routine can eliminate these tracks, but the tracks are atill lost. The CPU time required to use the methods of the track identification routine to recover there events would be prohibitive. Rather than try to calculate the efficiency for confusion, and aubsequent removal, a cut is made requiring that the hits be separated by at least $2 \mathrm{~cm}$ in all chambers. Also, as pointed out in Section 3.3, if the hit separation is more than $2 \mathrm{~cm}$ the eficiency for reconatruction of Monte Carlo two track events is approximately constant (see Fig. 5.7).

These three cuts give a step-function two track efficiency - either the track is cut, or the two tracks are sufficiently well separated in all detectoru that the track finding efficiency is constant. These cuts are imposed on the real data and the background date at the stage where the data were histogrammed and at any point where the two track efficiency function $\left(\rho_{i j}\right)$ is used when calculating the event weights in the residual correlation removal (see Chapter 6). The Monte Carlo test of the correlation function fitting procedure (see Section 7.2) included these cuts.

\subsection{Particle Identification}

Particle identification in the two pion experiment wa done at a simple level. If the spectrometer is set to accept negative particles then all detected particles are assumed 
to be negative pions. The electron contamination was estimated by taking the number of $\pi^{\circ}$ 's produced to be equal to the number of $\pi^{-1}$ 's produced, taking all of the $\pi^{0 \text { 's }}$ as decaying into $2 \gamma$ 's, which are asnumed to continue at the momentum of the $\pi^{\circ}$ (this will overestimate the number of high energy electrons) and then the conversion probability for the $\gamma^{\prime} / 3$ was calculated for the target and the air up to the first counters. The particle identification in the negative data is estimated to give an electron contamination of about $3 \%$ or less.

\subsection{Momentum and Other Track Parameter Fitting}

After the particles have been identified, a better track reconstruction is done.

First, any misning wires in the track are reconatructed uning equations derived from Eq. 3.8. The tracks' parameters are then calculated a functions of the measured quantities, namely the wire numbers. The procedure used is to use a Monte Carlo simulation to generate a large number of tracks to give the wire numbers hit in the wire chambers as a function of the initial position of the particle and its vector momentum. Note that since there are twelve planes of wires and only five free parameters (two for the initial position at the target plane and three for the vector momentum) some of the wire chamber data are redundant. There is sufficient redundancy that the fourth wire chamber is removed from the input to the fits and later used to verify the effective-edge track reconstruction. The remaining wire chamber data (nine planes) are subject to a principle component analysis[43] to determine which linear combinations of the wire numbers show the most variation in the Monte Carlo data set and which show the least. The five linear combinations showing the most variation were considered to be the most significant to the fit of the parameters as a function the wire numbers, and the remaining four combinations redundant. Each of the desired parameters was then fit using orthogonal polynomials as a function of the five retained wire number combinationa. In this experiment Chebyshev polynomials were used aince they have good properties for interpolating data. The program used for this analysis was ExIKA written by Harald von Fellenberg and modified by Jim Miller and William Zajc to run on the computers ured.

The input data for this procedure is a Monte Carlo aimulation of the experiment. The Monte Carlo program is described in Appendix A. We used Monte Carlo data with 
multiple scattering and Landau straggling to determine the momentum resolution of this procedure and the results are given in Table 5.2. The coordinate system used is: $-\hat{y}$ is along the beam line, $\vec{z}$ is vertical, and $\hat{x}$ is parpendicular to the beam. Note that $\vec{p}$ was calculated in the laboratory frame and $\mathbf{Q}$ was calcidated in the center of mass (of the nuclei) frame.

\begin{tabular}{|c|c|}
\hline Quantity & RMS error \\
\hline$p_{s}$ & $3.6 \mathrm{MeV} / \mathrm{c}$ \\
$p_{v}$ & $2.2 \mathrm{MeV} / \mathrm{c}$ \\
$p_{s}$ & $3.4 \mathrm{MeV} / \mathrm{c}$ \\
\hline$q_{s}$ & $5.1 \mathrm{MeV} / \mathrm{c}$ \\
$q_{v}$ & $1.7 \mathrm{MeV} / \mathrm{c}$ \\
$q_{s}$ & $4.8 \mathrm{MeV} / \mathrm{c}$ \\
$q_{0}$ & $1.8 \mathrm{MeV}$ \\
\hline
\end{tabular}

Table 5.2: Resolution for Monte Carlo simulated events.

From the date of Table 5.2 the resolution in the components of $\left(\vec{q}, q_{0}\right)$ histogrammed is computed to be (using $\Delta$ to denote the uncertsinty in a quantity) $\Delta q_{0}=2 \mathrm{MeV}$, $\Delta q_{1}=2 \mathrm{MeV} / \mathrm{c}$ and $\Delta q_{\perp}=7 \mathrm{MeV} / \mathrm{c}$. The momentum differences were histogrammed over a raage of $0-250 \mathrm{MeV} / \mathrm{c}$ (or $\mathrm{MeV}$ for 90 ) with a bin width of $10 \mathrm{MeV} / \mathrm{c}$ (or MeV). The apectrometer's sesolvtion is eatimated to change the value of the correlation function in the bins of the histogram by < $1 \%$ (FWHM). The range of the parameters resolvable by the apparatus was eatimated by requiring that the correlation function vary by $e^{-1 / 2}$ over the full range of the histograms, and from the first bin to the second bin. The range over which $R_{\perp}, R_{\|}$and cr are resolvable is from $\sim 1$ to $\sim 20 \mathrm{fm}$.

In addition to the momentum, other track parameters were fit using the principle component analysis. The parameters are: the target traceback, the distance to the lead walls in the $\mathbf{C}$ magnet, and the position of the hit in the fourth wire chamber. One can derive from the individual target tracebacks the separation between the two tracks at the target (which should be zero if the two tracks come from the same nuclear collinion). These quantitiea were compared to the expected values to verify that the track was reconstructed

\footnotetext{
"These valwea imply that the histograna have 25 bies on each side.
} 
correctly. These cuts were typically placed at three times the corresponding RMS error as calculated frum the Monte Carlo tracks. 


\section{Chapter 6}

\section{Background Generation and Function fitting}

\subsection{Background Event Generation}

The word "background" is used $h_{\text {ine }}+$ mean a two pion data sample with all the physics incorporated except for the Bose-Einstein symmetrine.ion. The measured correlation function is then the ratio of the rea data to the background data. It is vital that the single and two track efficienciea be incorporated currectly so that when the ratio of the real data and the background data is taken the efficiencies will cancel.

In this experiment the method of event mixing was used to generate the background data sample. Event mixing consists of taking one pion from an event, adding to it one pion from a different, ranciomly chosen, event and calling this a two picn event, which is then used to form the background. This assumes that the momentum distribution of a pion in the two pion event is not influenced by the other pion in the event (the ignored pion). Since the pions are correlated (measurement of this correlation is the subject of this thesis), this is only approximately true. The background events are corrected for the correlation by weighting the pions that are mixed to form the event. To show how this weighting is done and, how the background events are generated, it is necessary to consider in detail how the correlation function is calculated from the two pion data. This requires the calculation of the "residual correlation" for the background histogram bins. 
The reajaal correlation calculation is then cast as an event weighting calculation, and the momentum binning assumed no longer appears.

Once the background events have been generated, the correlation function is fit to the measured and the background data samplea. The Principle of Maximum Likelihood was uned when fitting the data, to avoid biasing the data when the number of counts per bin is small and Poisson atatiatics must be used. Two different measures of the quality of the fit are given, that of a restricted $x^{2}$, and that of a generalized $\chi^{2}$, denoted $\chi_{\text {FuL }}^{2}$, which is derived from the Principle of Maximum Likelihood and is based on the log of the likelihood function.

\subsection{Derivation of Event Mixing}

This section uses notation based on the notation uned in [1]:

Let $\boldsymbol{\eta}_{i}$ be the one particle detection efficiency.

$\eta_{i j}$ be the two particle detection efficiency.

$\omega_{i}$ be the number density of emitted particles.?

$C_{i j}$ be the Bone-Einatein correlation function.

$\rho_{i j}$ be the two particle contribution to the detection efficiency, defined such that $\eta_{i j}=\eta_{i} \eta_{j} \rho_{i j}$.

In the above $i, j$ represent momentum bins; That is $i \rightarrow \vec{p}_{i}$ and $j \rightarrow \vec{p}_{j}$ and so on. Note that [1] asumed that $\eta_{i j}=\eta_{i} \eta_{j}$, or $\rho_{i j} \equiv 1$. Although only one dimension of the histograms will be shown explicitly, it is simple to extend to arbitrary numbers of dimensions by adding subscripts. ${ }^{2}$ The correlation function, $C_{i j}$, will not be taken to be an explicit function of $\vec{q}$. Since $\vec{q}=\vec{p}_{1}-\vec{p}_{2}$, one can regard $C(\vec{q})$ as $C\left(\vec{q}\left(\vec{p}_{1}, \vec{p}_{2}\right)\right)=C_{i j}$ without low of generality.

The lettess $i, j, k, \ldots$ will be uned for sums over momentum bins and $\alpha, \beta, \gamma, \ldots$ for sums over events. The calligraphic letter $\mathcal{P}$ will be uned for probability. In the two pion

\footnotetext{
'Note the tochnique of event mixins semen that a single aumber dencity of emittod particlen, wi, describes all the eventu. Section 3.3 diecum some of the concequences of this ammption. See aleo [37] for a discruiton of this and related points.

${ }^{2}$ One can aloo number all the biso sequentially in some fanhion and we the equations derived here.
} 
events there are two momenta, so $\vec{p}_{a 1}$ will be used for the $\alpha^{\text {th }}$ event's first pion momentum and $\vec{p}_{\beta 2}$ for the $\beta^{\text {th }}$ event's second pion momentum.

With this notation, the two pion experiment consists of measuring a set of $N$ two pion events, which can be written

$$
\left\{\left(\vec{p}_{a 1}, \vec{v}_{0}-\right)\right\}_{a=1}^{N} \text {, where } N=\text { the number of events. }
$$

Central to the analysis is making a histogram, so the definition of a function that corresponds to making a hintogram is ueeded. Therefore, define a $\delta$ function by

$$
\delta(p \in i)=\left\{\begin{array}{l}
1 \text { for } \vec{p} \in \text { ith momentum bin } \\
0 \text { for } \vec{p} \notin \text { ith momentum bin }
\end{array}\right.
$$

then making a histogram of the momentum distribution for the first pion in the eventa corresponds to finding the $n_{i}$ that are the number of counts per bin by

$$
n_{i}=\sum_{\alpha=1}^{N} \delta\left(\tilde{p}_{\alpha 1} \in i\right)
$$

Before starting into the details of the calculations, it is best to ieveal the plan of attack. What is wanted is, of course, the $C_{i j}$ in terms of known quantities. The known quantities in this experiment are the momentum distributions of the pions, the $\delta\left(\vec{p}_{a z} \in i\right)$, for the data set of two pion events. The functions $\eta_{i}, w_{i}$ and $\rho_{i j}$ are unknown (or, in the case of $\rho_{i j}$, known approximately). Therefore, the calculation will express the $\eta_{i}$, $\omega_{i}$ and $C_{i j}$ in terms of the momentum distributions and other known quantities. This : equires that some normalizations be assumed for the probabilities and for the momentum distributions. In many cases the value assumed for the normalization is unimportant, so long as a normalization is assumed.

Assume the following normalizations

$$
\begin{aligned}
\sum_{i=1}^{M} \omega_{i} & =1, \text { Probsbility of emission somewhere }=1, \text { and } \\
\sum_{i} \eta_{i} \omega_{i} & =\Omega, \text { The efficiency of the spectrometer. }
\end{aligned}
$$

Since $\Omega$ is unknown, this can be used to define $\Omega$. Also, the normalization of $\omega_{i}$ implies that $\omega_{i} \sim \mathcal{O}\left(\frac{1}{k}\right)$, where $M=$ the number of bins. 
The normalization for the real two track probability with detector problems, $\mathcal{P}_{2}^{R}$ (the superscript $R$ is intended to denote a "real" momentum distribution, as opposed to the "fake" momentum distributions that will be considered later on), is found by considering the definition of $\mathcal{P}_{2}^{R}$,

$$
\begin{aligned}
\mathcal{P}_{2}^{R}(i, j) & \equiv \text { the real probability of a two track event with } \vec{p}_{1} \in \text { ith bin, } \vec{p}_{2} \in j \text { th bin } \\
& =\eta_{i} \omega_{i} \eta_{j} \omega_{j} C_{i j} p_{i j} .
\end{aligned}
$$

It is clear that,

$$
p_{2}^{R}(i, j) \propto \sum_{a} \delta\left(p_{a 1} \in i\right) \delta\left(p_{a 2} \in j\right) .
$$

To find the constant of proportionality use

$$
\sum_{i j} \sum_{a} \delta\left(\vec{p}_{\alpha 1} \in i\right) \delta\left(\vec{p}_{\alpha i} \in j\right)=N .
$$

Note that if $C_{i j} \rho_{i j}=1 \forall i, j$, then ${ }^{3}$

$$
\begin{aligned}
\sum_{i j} \mathcal{P}_{2}^{R}(i, j) & =\sum_{i j} \eta_{i} \omega_{i} \eta_{j} \omega_{j} C_{i j} \rho_{i j} \\
& =\Omega^{2},
\end{aligned}
$$

so define $A$ such that

$$
\begin{aligned}
\sum_{i j} \mathcal{P}_{2}^{R}(i, j) & =\sum_{i j} \eta_{i} \omega_{i} \eta_{j} \omega_{j} C_{i j} \rho_{i j} \\
& =\Omega^{2} A .
\end{aligned}
$$

Then, using

$$
\frac{\Omega^{2} A}{N} \sum_{i j} \sum_{a} \delta\left(\vec{p}_{\alpha 1} \in i\right) \delta\left(\vec{p}_{\alpha 2} \in j\right)=\Omega^{2} A
$$

the normalization desired can be seen to be

$$
\mathcal{P}_{2}^{R}(i, j)=\frac{\Omega^{2} A}{N} \sum_{a} \delta\left(\vec{p}_{a 1} \in i\right) \delta\left(\vec{p}_{a 2} \in j\right) .
$$

The above definition can be seen to be equivalent to

$$
\begin{aligned}
A & =\frac{\sum_{i j} C_{i j} \rho_{i j} \eta_{i} \omega_{i} \eta_{j} \omega_{j}}{\sum_{i} \eta_{i} \omega_{i} \sum_{j} \eta_{j} \omega_{j}} \\
& =\frac{\text { The number of detected two track events }}{\text { The number of two track events detected, if seen one track at a time }}
\end{aligned}
$$

\footnotetext{
${ }^{3}$ The symbol " $\gamma^{n}$ is uned to denote the phrace "for all".
} 
Note that $A$ is unknown unless the $C_{i j}$ and $\rho_{i j}$ are known.

Now, to find the correlation function, use the defin'tions (Eqs. 6.4, and 6.10) of $\mathcal{P}_{2}^{R}$, to get

$$
\eta_{i} \omega_{i} \eta_{j} \omega_{j} C_{i j} \rho_{i j}=\frac{\Omega^{2} A}{N} \sum_{\alpha} \delta\left(\vec{p}_{\alpha 1} \in i\right) \delta\left(\vec{p}_{\alpha 2} \in j\right)
$$

thus

$$
\sigma_{i j}=\frac{\frac{n^{2} A}{N} \sum_{\alpha} \delta\left(\vec{p}_{\alpha 1} \in i\right) \delta\left(\vec{p}_{\alpha 2} \in j\right)}{\eta_{i} \omega_{i} \eta_{j} \omega_{j} \rho_{i j}} .
$$

At this point the task of calculating the correlation function, $C_{i j}$, is half complete. The correlation function has been expressed in terms of the two particie momentum distribution, the $\eta_{i}$, the $\omega_{i}$ and $\rho_{i j}$. It will turn out to be impossible to eliminate $\rho_{i j}-$ the two particle contribution to the detection efficiency - it will be approximated by the cuts outlined in Chapter 5. These cuts will be applied to the real data (to give an explicit form for $\rho_{i j}$ ) and whenever $\rho_{i j}$ appears in a formula.

Now to continue the calculation of the correlation function, the $\eta_{i} \omega_{i}$ are needed. The $\eta_{i} \omega_{i}$ are just the real single track probability of detection (which will be denoted $\mathcal{P}_{1}^{R}$ ). It is expected that, since the correlation is not strong, the two pions in the event will behave approximately independently. So the single pion detection probability can be approximated by just ignoring one of the two pions in the two pion event. So define $\mathcal{P}_{\mathbb{1}}^{F}$ to be the "fake" one track probability, that is, the probability of detection for one track without regard to the second track of the pair in the event. Roughly,

$$
\mathcal{P}_{1}^{F}(i)=\frac{1}{N} \sum_{\alpha} \delta\left(\vec{p}_{\alpha 1} \in i\right), \text { where } \vec{p}_{\alpha 2} \text { is ignored. }
$$

The normalization for $\mathcal{P}_{\mathfrak{l}}^{F}$ is given by considering

$$
\mathcal{P}_{1}^{F}(i) \propto \eta_{i} \omega_{i} \sum_{m} \eta_{m} \omega_{m} C_{i m} \rho_{i m},
$$

where the sum is just the probability of observing the first pion's at momentum $i$, summed over all possible values tor the second pion's momentum. If $C_{i m} p_{i m}=1 \forall i, m$, then

$$
\eta_{i} \omega_{i} \sum_{m} \eta_{m} \omega_{m} C_{i m} \rho_{i m}=\eta_{i} \omega_{i} \Omega
$$

\footnotetext{
The firat track will be used to generate the background. This in not emential since the anigament of the tracke should be random. The computer codes pick the tracke randomly from the firat and recond track is the events to a: -id biaces in the track-finding programs.
} 
Wheres, if there are no two pion effects, so $C_{i j} \rho_{i j}=1 \forall i, j$, then $\mathcal{P}_{1}^{F}(i)=\eta_{i} \omega_{i}=$ $\mathcal{P}_{1}^{R}(i)$ (that appears in the denominator of Eq. 6.14 above). Therefore, a convenient normalization is

$$
\mathcal{P}_{1}^{F}(i)=\eta_{i} \omega_{i} \frac{1}{\Omega} \sum_{m} \eta_{m} \omega_{m} C_{i m} \rho_{i m}
$$

Now, write

$$
P_{i}^{F}(i)=\sum_{m} \frac{1}{\Omega} \eta_{i} \omega_{i} \eta_{m} \omega_{m} C_{i m} p_{i m}
$$

and use Eq. 6.13 for $\eta_{i} \omega_{i} \eta_{m} \omega_{m} C_{i m} \rho_{i m}$. Then

$$
P_{i}^{F}(i)=\frac{\Omega A}{N} \sum_{m} \sum_{a} \delta\left(\vec{p}_{a 1} \in i\right) \delta\left(\vec{p}_{a 2} \in m\right) .
$$

Interchange the $m, \alpha$ sume and un

$$
\sum_{m} \delta\left(\vec{p}_{a 2} \in m\right)=1 \text {, since } \vec{p}_{a 2} \text { must be somewhere, }
$$

then the normalization for $P_{1}^{F}(i)$ is given by

$$
\mathcal{P}_{1}^{F}(i)=\frac{\Omega A}{N} \sum_{a} \delta\left(\bar{p}_{a 1} \in i\right) .
$$

Use both expressions for $\mathcal{P}_{1}^{F}(i)$ (Eqs. 6.17, and 6.21) to get

$$
\frac{\Omega A}{N} \sum_{a} \delta\left(\vec{p}_{a 1} \in i\right)=\eta_{i} \omega_{i} \frac{1}{\Omega} \sum_{m} \eta_{m} \omega_{m} C_{i m} \rho_{i m}
$$

and so,

$$
\eta_{i} \omega_{i}=\frac{\not A}{\frac{\not}{\hbar} \sum_{a} \delta\left(\vec{p}_{a 1} \in i\right)} \frac{1}{\hbar \sum_{m} \eta_{m} \omega_{m} C_{i m} \rho_{i m}}
$$

and the $\eta_{i} \omega_{i}$ have been expressed in terms of a momentum distribution and a weighting factor.

This is a formal solution to finding the correlation function in that Eq. 6.23 gives us the $\eta_{i} w_{i}$ and Eq. 6.14 gives us $C_{i j}$ in terms of the $\eta_{i} w_{i}$ and known (or measurabie) quantities.

To examin the role of the weighting factor more carefully, set

$$
W_{i}=\frac{1}{\hbar \sum_{m} \eta_{m} \omega_{m} C_{i m} \rho_{i m}}
$$

then combining Eq3. 6.14 and 6.23 gives

$$
C_{i j}=\frac{\frac{\Omega^{2} A}{d} \sum_{a} \delta\left(\bar{p}_{a 1} \in i\right) \delta\left(\bar{p}_{a 2} \in j\right)}{\left(\frac{\phi A}{N}\right)^{2} W_{i} W_{j} \rho_{i j} \sum_{\rho} \sum_{\gamma} \delta\left(\vec{p}_{\beta 1} \in i\right) \delta\left(\vec{p}_{\gamma 1} \in j\right)}
$$


and it in apparent that $C_{i j}$ is the ratio of the real data to a background data sample that is made by combining pions from different events. Another important feature is the fact that the $\eta_{i} \omega_{i}$ do not appear in the calculation of $C_{i j}$. The $\eta_{i} \omega_{i}$ in the numerator sancel those in the denominator and, as a result, measuring the spectrometer's single track efficiency is unnecessary. This is an extension of Eq. 3.15 in that the two track efficiency is included in the denominator.

The origin of the weighting factor is thin: even though only one pion is used, there was another pion detected in the event. If there were no correlation between the pions then the probability distributiou for the iguored pion would be $\eta_{m} \omega_{m}$ (if $m$ is its momentum bin), and the ignored pion would just weight, on average, the kept pion by $\sum_{m} \eta_{m} \omega_{m}$, a constant. But the pions are correlated 00 the probability distribution for the ignored pion, given that the kept pion has momentum $i$, is $\eta_{m} \omega_{m} C_{i m} \rho_{i m}$ (which, when aummed over the momentum bins, gives a weight of $\left.\sum_{m} \eta_{m} \omega_{m} C_{i m} \rho_{i m}\right)$ and the kept pion, through the correlation function, modifes the probability distribution of the ignored pion. If the modified probability distribution happens to increase the probability of the ignored pion being in.regions where the detection efficiency is high, then the probability of detecting the kept pion is increased (relative to the case where there is no correlation). The combination of the probability of emission $\left(\eta_{m}\right)$, the probability of detection $\left(\omega_{m}\right)$, the two particle contribution to the detector efficiency $\left(p_{i m}\right)$, and the corralation function $\left(C_{i m}\right)$ all contribute to the weighting factor.

\subsection{Residual Correlations}

Define "renidual correlation" to be the ratio between the fake single track probability and the real single track probability, that is $\mathcal{P}_{i}^{F} / \mathcal{P}_{1}^{R}$. This is just the reciprocal of the weight defined in Eq. 6.24. From Eq. 6.17 this can be seen to be

$$
\frac{\mathcal{P}_{1}^{F}(i)}{\mathcal{P}_{1}^{R}(i)}=\frac{1}{\Omega} \sum_{m} \eta_{m} \omega_{m} C_{i m P_{i m}} .
$$

For an ideal spectrometer, $\eta_{m}=1 \forall m$, and $\rho_{\text {im }}=1 \forall i, m$, then

$$
\frac{\mathcal{P}_{i}^{F}(i)}{\mathcal{P}_{i}^{R}(i)}=\frac{1}{\Omega} \sum_{m} \omega_{m} C_{i m},
$$


which is not necessarily equal to one. In principle, it should be necessary to correct for residual correlations for perfect spectrometers, since the emission probability can induce a residual correlation. For $24 \pi$ spectrometer with (only) two track resolution problems, $\rho_{\text {im }} \neq 1$, and

$$
\frac{\mathcal{P}_{1}^{F}(i)}{\mathcal{P}_{1}^{R}(i)}=\frac{1}{\Omega} \sum_{m} \omega_{m} C_{i m} P_{i m} .
$$

So revidual correlations could be important.

\subsection{Iteration Scheme for the Event Weights}

To calculate the correlation function, a pract:eal way to calculate the $\eta_{i} \omega_{i}$ is needed, so expand Eq. 6.23 in a amall parameter. It will prove to be possible to derive a succestive approximation scheme in powern of a suitably chosen small parameter. The expansion used here is motivated by noting that the correlation between pions is not expected to be strong. This implies that the effect of the ignored pion will not be large, or

$$
\mathcal{P}_{i}^{R}(i) \approx \mathcal{P}_{i}^{F}(i)
$$

which is equivalent to

$$
\eta_{i} \omega_{i} \approx \frac{\Omega A}{N} \sum_{a} \delta\left(\bar{p}_{a 1} \in i\right)
$$

which is equivalent to, from Eq. 6.23,

$$
\frac{1}{\Omega} \sum_{m} \eta_{m} \omega_{m} C_{i m \rho_{i m}} \approx 1 \text {. }
$$

So, write

$$
\frac{1}{\Omega} \sum_{m} \eta_{m} \omega_{m} C_{i m} \rho_{i m}=1+\frac{\delta_{i}}{\Omega} .
$$

Since $\Omega \leq 1, \delta_{i}$ is still a small number then, using $\frac{1}{\Omega} \sum_{m} \eta_{m} \omega_{m}=1$,

$$
\frac{1}{\Omega} \sum_{m} \eta_{m} \omega_{m} C_{i m} \rho_{i m}=\frac{1}{\Omega}\left(\sum_{m} \eta_{m} \omega_{m}+\delta_{i}\right) \text {. }
$$

Define $\delta_{\text {im }}$ such that

$$
\frac{1}{\Omega} \sum_{m} \eta_{m} \omega_{m} C_{i m} \rho_{i m}=\frac{1}{\Omega} \sum_{m} \eta_{m} \omega_{m}\left(1+\delta_{i m}\right),
$$

a suitable such $\delta_{i m}$ is $\delta_{i m}=C_{i m} \rho_{i m}-1$. If this is used, then $\delta_{i}=\sum_{m} \eta_{m} \omega_{m} \delta_{i m}$ (from Eq. 6.29 and 6.30 ) and, on the average, $\delta_{i m}$ is small. 
With this as motivation, take $\delta_{i m}=C_{i m} \rho_{i m}-1$ and expand Eq. 6.22 in powers of $\delta_{\text {im }} \cdot{ }^{5}$ Recall Eq. 6.22:

$$
\frac{\Omega A}{N} \sum_{\alpha} \delta\left(\bar{p}_{\alpha 1} \in i\right)=\frac{\eta_{i} \omega_{i}}{\Omega} \sum_{m} \eta_{m} \omega_{m} C_{i m} P_{i m}
$$

Use $C_{i m} \rho_{i m}=1+\delta_{i m}$ and $\frac{1}{n} \sum_{m} \eta_{m} \omega_{m}=1$, then

$$
\frac{\Omega A}{N} \sum_{\alpha} \delta\left(\vec{p}_{\alpha 1} \in i\right)=\eta_{i} \omega_{i}\left(1+\frac{1}{\Omega} \sum_{m} \eta_{m} \omega_{m} \delta_{i m}\right)
$$

and

$$
\eta_{i} \omega_{i}=\frac{\eta A \sum_{a} \delta\left(\bar{p}_{a 1} \in i\right)}{1+\hbar \sum_{m} \eta_{m} \omega_{m} \delta_{i m}} .
$$

So far there have been no approximations. Note that $\eta_{m} \omega_{m}$ appears as $\sum_{m} \eta_{m} \omega_{m} \delta_{i m}$, so $\eta_{m} \omega_{m}$ can be accurate to the $(n-1)^{\text {th }}$ order in $\delta_{i m}$, but the equation will still be $n^{\text {th }}$ order in $\delta_{\text {im. }}$. This suggests the following approximation scheme be used

$$
\begin{aligned}
& \left(n_{i} \omega_{i}\right)^{0 \mathrm{ch}}=\frac{\Omega A}{N} \sum_{a} \delta\left(\vec{p}_{a 1} \in i\right) \\
& \left(\eta_{i} \omega_{i}\right)^{n+h}=\frac{\not A \sum_{a} \delta\left(\vec{p}_{a 1} \in i\right)}{1+\frac{1}{R} \sum_{m}\left(\eta_{m} \omega_{m}\right)^{(n-1) t h \delta_{i m}}} .
\end{aligned}
$$

Note that Eq. 6.33 results from assuming that $\delta_{i m}=0$. The problems with this idea are that (1) $A$ is unknown, and (2) $\delta_{\text {im }}$ will be hard to calculate. However, having found the approximation scheme, the $\delta_{\text {im }}$ can be eliminated and a condition to give $A$ can be found.

First, the condition to give $A$ will be found. Note that $\frac{1}{\hbar} \Sigma_{i} \eta_{i} \omega_{i}=1$. Calculate this sum using the $0^{\text {th }}$ order equation (Eq. 6.33), to get

$$
\begin{aligned}
\sum_{i} \eta_{i} \omega_{i} & =\frac{\Omega A}{N} \sum_{i} \sum_{\alpha} \delta\left(\vec{p}_{\alpha 1} \in i\right) \\
\Omega & =\frac{\Omega A}{N} \cdot N
\end{aligned}
$$

and $A=1$ to $0^{\text {th }}$ order of $\delta_{i m}$. Hence

$$
\left(\eta_{i} \omega_{i}\right)^{a h}=\frac{\Omega}{N} \sum_{a} \delta\left(\vec{p}_{a 1} \in i\right)
$$

and at each step one can $f_{i x} A$ by the requirement that $\sum_{i} \eta_{i} \omega_{i}=\Omega$.

\footnotetext{
${ }^{5}$ Note that ie small parameter, $\delta_{i m}$, is not directly related to the histogram making function, $\delta\left(\vec{p}_{a 1} \in i\right)$.
} 
The $\delta_{i m}$ can then be eliminated through use of the definition $\delta_{i m}=C_{i m} \rho_{i m}-1$ and noting that $\frac{1}{\delta} \sum_{m} \eta_{m} \omega_{m}=1$. With these, the iteration scheme for calculating the $\eta_{i} \omega_{i}$ becomes

$$
\begin{aligned}
\left(\eta_{i} \omega_{i}\right)^{\alpha \text { th }} & =\frac{\Omega}{N} \sum_{a} \delta\left(\vec{p}_{\alpha 1} \in i\right) \\
\left(\eta_{i} \omega_{i}\right)^{\text {nth }} & =A \frac{f \sum_{a} \delta\left(\vec{p}_{\alpha 1} \in i\right)}{\hbar \sum_{m}\left(\eta_{m} \omega_{m}\right)^{(n-1) \text { th } C_{i m} P_{i m}}}
\end{aligned}
$$

where in Eq. 6.39, $A$ is chosen 20 that $\sum_{i}\left(\eta_{i} w_{i}\right)^{\text {nth }}=\Omega$.

Now the iteration scheme for the bin weights is converted into something easier to calculate on an event by event basis. This is desirable aince it is easier to deal with event weights because the histogram binning does not appear in the calculation of the event weights. The bin weighting scheme is recast into an event weighting scheme by using the fact that for small momentum bins (and continuous functions $F$ )

$$
\sum_{a} \delta\left(\vec{p}_{a 1} \in i\right) F\left(\vec{p}_{i}\right)=\sum_{\alpha} \delta\left(\vec{p}_{a 1} \in i\right) F\left(\vec{p}_{a 1}\right)
$$

where the difference is that $F$ is evaluated at the center of the momentum bin in the first case, and at the momentum of the pion in the event in the second case. Note that if histogramming is to have any merit in this anslysie, the momentum bins must be small enough so that this is true. The $\eta_{a} w_{a}$ (the event probabilities for detection and emission) will be defined so that

$$
\sum_{a} \eta_{a} \omega_{a} \delta\left(\vec{p}_{a 1} \in i\right)=\eta_{i} \omega_{i}
$$

then the correlation function, Eg. 6.14, becomes

$$
C_{i j}=\frac{\frac{\Omega^{2} A}{N} \sum_{a} \delta\left(\vec{p}_{a 1} \in i\right) \delta\left(\vec{p}_{a 2} \in j\right)}{\sum_{\rho} \delta\left(\vec{p}_{\beta 1} \in i\right) \eta_{\beta} \omega_{\beta} \sum_{\gamma} \delta\left(\vec{p}_{\gamma 1} \in j\right) \eta_{\gamma} \omega_{\gamma} p_{\beta \gamma}} .
$$

A suitable set of such probabilities can be seen to be

$$
\begin{aligned}
& \left(\eta_{\alpha} \omega_{\alpha}\right)^{\alpha \mathrm{h}}=\frac{\Omega}{N}
\end{aligned}
$$

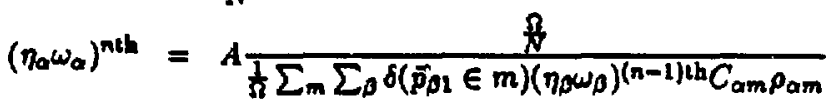

since the addition of the sum over events and the $\delta$ functions in Eq. 6.42, and the removal of the same from 6.43, and 6.44, reproduces Eqs. 6.14, 6.38 and 6.39 (using Eq. 6.40, and 
using Eq. 6.41 to change from $\eta_{\beta} \omega_{\beta}$ to $\eta_{m} \omega_{m}$ in Eqs. 6.42, 6.44). Interchanging the $m$ and $\beta$ sums and uaing Eq. 6.40 gives

$$
\begin{aligned}
& \left(\eta_{a} \omega_{a}\right)^{\text {alh }}=\frac{\Omega}{N} \\
& \left(n_{\alpha} \omega_{\alpha}\right)^{\text {nch }}=A \frac{\frac{R}{h}}{\frac{1}{h} \sum_{\alpha}\left(\eta_{\alpha \omega_{\beta}}\right)^{(n-1) \text { th }} C_{\alpha \rho} \rho_{\alpha \beta}} .
\end{aligned}
$$

The normalization condition for $A$ in Eq. 6.46, is found from Eq. 6.41 and using Eq. 6.20, with these

$$
\begin{aligned}
\Omega & =\sum_{i}\left(\eta_{i} \omega_{i}\right)^{\text {nth }} \\
& =\sum_{i} \sum_{j} \delta\left(\vec{p}_{\beta 1} \in i\right)\left(\eta \omega_{\beta}\right)^{\text {nth }} \\
& =\sum_{j}\left(\eta \omega_{\beta}\right)^{\text {nth }} .
\end{aligned}
$$

Finally, to get the event weights and eliminate the normalization of the $\eta_{a} \omega_{\alpha}$, set $W(\alpha)=\frac{2 \alpha_{\alpha} \alpha}{n}$. Than Eq. 6.42 becomes

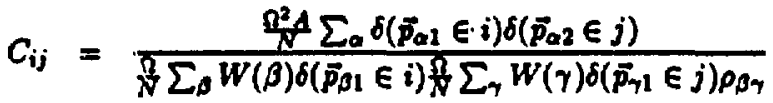

$$
\begin{aligned}
& =\frac{A N \sum_{a} \delta\left(\vec{p}_{o 1} \in i\right) \delta\left(\vec{p}_{a 2} \in j\right)}{\sum_{\beta} W(\beta) \delta\left(\vec{p}_{\beta 1} \in j\right) \sum_{\gamma} W(\gamma) \delta\left(\vec{p}_{\gamma 1} \in j\right) p_{\beta \gamma}}
\end{aligned}
$$

and the iteration scheme becomes

$$
\begin{aligned}
& (W(\alpha))^{\alpha \text { th }}=1 \\
& (W(\alpha))^{\text {nth }}=A \frac{1}{\sqrt{W} \sum_{\beta}(W(\beta))^{(n-1) \text { th }} C_{a \beta \rho_{\alpha \beta}}}
\end{aligned}
$$

where, in Eq. 6.50, the normalization condition is that $A$ is chosen such that

$$
\sum_{\beta}(W(\beta))^{\text {nth }}=N
$$

or the average weight is one.

At this point the task is complete, $C_{i j}$ has been expreased in terms of momentum dis. tributions (and some constants) with event weights, and the event weights are expressed as 2 succeasive approximation scheme involving the correlation function and the two particle contribution to the spectrometer's efficiency. All that remains is to describe how 
the correlation function is fit, and how to convert these equations to the ones used in the computer codes.

In the actual computer codes, the normalization of $C_{i j}$ is fit. Therefore, in Eq. 6.48, $A N$ is only estimated by setting it equal to the inverse of the ratio of the total number of real events to the total number of background events, where real and background are defined below. Also, due to problems associated with taking the ration of small numbers[44] that renult from the moderate-sized data samples available, the value of $C_{i j}$ is not calculated. Rather, one chooces $C_{i j}$ to maximize the agreement in this equation

$$
R_{i j}=C_{i j} \cdot B_{i j}
$$

where the $R_{i j}$ are the real events per bin and the $B_{i j}$ are the background, or fake, events per bin. These are given by

$$
\begin{aligned}
& R_{i j}=\sum_{\alpha} \delta\left(\vec{p}_{\alpha 1} \in i\right) \delta\left(\vec{p}_{\alpha 2} \in j\right) \\
& B_{i j}=\frac{1}{A N} \sum_{\beta} \sum_{\gamma} W(\beta) W(\gamma) \rho_{\beta \gamma} \delta\left(\vec{p}_{\beta 2} \in i\right) \delta\left(\vec{p}_{\gamma 2} \in j\right)
\end{aligned}
$$

since the ratio of $R_{i j}$ and $B_{i j}$ is just Eq. 6.48.

\subsection{Momentum Difference (Q) Histograms}

For convenience, the calculation has used momentum bins to derive the residual correlation and the event weighting used to eliminate it. However, now that this has been done, there is no longer any explicit reference to the binning assumed. Therefore the histogramming assumed in Eqs. 6.33 and 6.54 can be changed without effecting the validity of the event weighting.

Recalling derivation of the correlation function (Chapter 3), the natural variables to use are $q_{\perp}, q_{1}$ and $q_{0}$, the components of the momentum difference of the pions, and the energy difference. Taking one index for each of $q_{\perp}$, qा and $q_{0}$, the natural enumeration of the histogram bins" in a triplet of indices, $i j k$. So now letting $i j k$ stand for the $i j k^{\text {th }}$ $\mathbf{Q}$ bin and using the four vector $\mathbf{Q}$ explicitly (and adding the normalization factor $D$ )

\footnotetext{
"This experiment und hiatograme 25 bins on each of the three sides. The bin width was $10 \mathrm{MeV} / \mathrm{c}$ (or MeV, for $\boldsymbol{\varphi}$ ).
} 
Equations 6.52, 6.53 and 6.54 become

$$
\begin{aligned}
& R_{i j k}=D \cdot C_{i j k} \cdot B_{i j k} \\
& R_{i j k}=\sum_{a} \delta\left(\left\{\mathbf{P}_{\alpha 1}-\mathbf{P}_{\alpha 2}\right\} \in i j k\right) \\
& B_{i j k}=\sum_{\beta} \sum_{\gamma} W(\beta) W(\gamma) \rho_{\beta \gamma} \delta\left(\left\{\mathbf{P}_{\Delta 1}-\mathbf{P}_{\gamma 1}\right\} \in i j k\right) \\
& \text { where } i j k \text { refers to the } i j k^{\text {th }} Q \text { bin. }
\end{aligned}
$$

The $W(\beta)$ and $W(\gamma)$ are calculated according to Eq. 8.49 and 6.50, where the overall normalization $(A)$ in the last iteration of the weights is ignored. This is done for the sake of computias apend; Since the normalization of the $C_{i j h}$ is fit this changen only the value of the normalization and doee not change the value of any of the physically significant parameters.? The form for $C_{i j h}$ used is that of Eq. 3.14, where $q_{\perp}, q_{\mid}$and $q 0$ are evaluated at thas center of the $i k^{\text {th }} Q$ bin.

\subsection{Background Fluctuations}

The number of counts in the background histogram is defined by Eq. 6.57

$$
B_{i j k}=\sum_{\rho} \sum_{\gamma} W(\beta) W(\gamma) p_{\beta \gamma} \delta\left(\left\{P_{\rho z}-P_{\gamma 1}\right\} \in i j k\right) \text {. }
$$

Because of the weight factors, the statistical fluctuation in the bins is not just the square root of the number of counts. Regarding each event as a bin with one count in it by taking the uncertainty in counting each weighted event as equal to the event weight, the estimated uncertainty in $B_{i j k}$ is (using $\Delta$ to denote the uncertainty in a quantity)

$$
\begin{aligned}
\left(\Delta B_{i j k}\right)^{2} & =\sum_{\beta}\left\{\sum_{\gamma} W(\beta) W(\gamma) p_{\beta \gamma} \delta\left(\left\{P_{\Delta_{1}}-P_{\gamma 1}\right\} \in i j k\right)\right\}^{2} \\
& +\sum_{\gamma}\left\{\sum_{\delta} W(\beta) W(\gamma) p_{\rho_{\gamma}} \delta\left(\left\{\cdots \gamma_{1}-P_{\gamma 1}\right\} \in i j k\right)\right\}^{2}
\end{aligned}
$$

where the two terms are due to each event appearing twice, once in the $\beta$ sum and once in the $\boldsymbol{\gamma}$ sum, in the function $\delta\left(\left\{P_{\rho 1}-P_{\eta 1}\right\} \in i j k\right)$. In the second term interchange

\footnotetext{
'While on the topic of computing speed, I sote that Eq. 6.50 and Eq. 6.57 both require donble loope over all events, heace go $\mathrm{m} N^{2}$. I do sot think that the $N^{2}$ behavior of Eq. 6.50 can be eliminated.
} 
the dummy summation labels, and use the fact that $\rho_{\beta \gamma}$ and $\delta\left(\left\{\mathbf{P}_{\beta 1}-\mathbf{P}_{\gamma 1}\right\} \in i j k\right)$ are symmetric functions, to find that

$$
\left(\Delta B_{i j k}\right)^{2}=2 \sum_{\beta}\left\{\sum_{\gamma} W(\beta) W(\gamma) \rho_{\rho_{\gamma}} \delta\left(\left\{\mathbf{P}_{\rho_{1}}-\mathbf{P}_{\gamma 1}\right\} \in i j k\right)\right\}^{2} .
$$

A plot of the fractional uncertainty $(\Delta B / B)$ is shown in Fig. 6.1. To eliminate those bins where the uncortainty is very large, a cut is made requiring that the fractional uncertainty be lese than $30 \%$. This cut is found to not change the fitted valuea at all, but improves the Principle of Maximum Llkelibood fit. Note that, for $N=$ the number of eventl, $\Delta B_{i j h} \sim O\left(N^{3 / 2}\right)$ and $B_{i j h} \sim O\left(N^{2}\right)$ so that the fractional error $\Delta B_{i j h} / B_{i j h} \sim$ $O\left(N^{3 / 4}\right)$.

\subsection{Other Corrections}

The discussion of the fitting procedures has ignored the Coulomb interactions of the pions with each other and with the nuclear fragments. These effects are handied as described in [1]. The formulas used there, and in this work, are from Gyulasy and Kaufimann[45] and the temperatures required for the formula come from the Nagamiya group $[39,40]$ and Sullivan et al.[46].

The pion-nuclear matter correction is handled by correcting the individual pion's momentum before histogramming, which does not alter any of the derivationa given above. This correction involves the Coulomb interaction between the pion and the target fragment, the source and the projectile fragment. The assumed distribution of the nuclear charge between the projectile fragment, the interaction region and the target fragment is $20 \%, 60 \%$ and $20 \%$, based on the average impact parameter. The pion-pion correction is handled by weighting the background events with the Gamow factor, ${ }^{*}$ to reflect the detection probability for \& pion pair with a given momentum difference. This introduces a weighting factor in the calculation of the background that would be placed in Eq. 6.57 next to the por term. This term in symmetric in the two pion momenta and the derivation of the uncertainty in $B_{i j k}$ can follow the one given above.

\footnotetext{
"See, for example, [47].
} 


$$
\begin{gathered}
1.54 \cdot \mathrm{A} \mathrm{GeV} \\
0^{9} \mathrm{Nb}+\mathrm{Nata}
\end{gathered}
$$

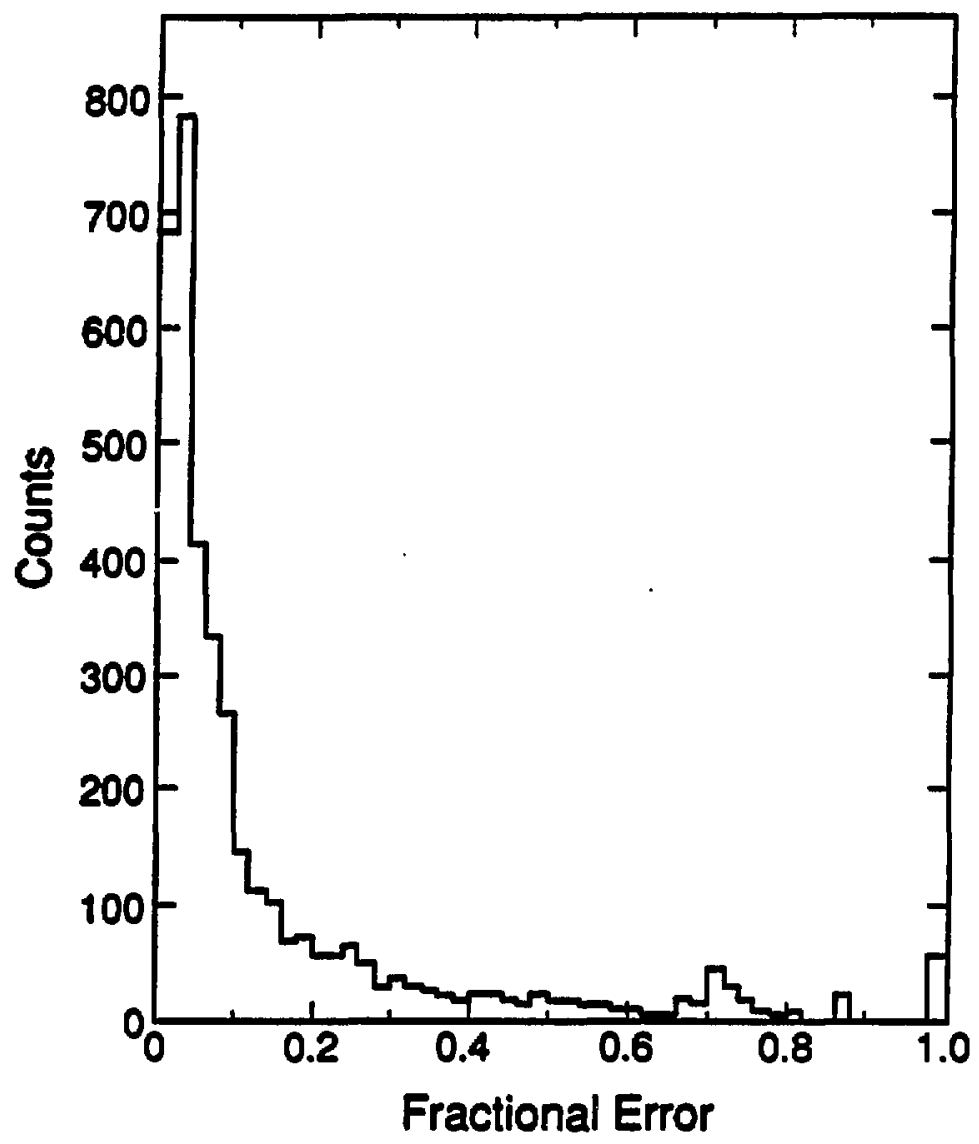

Figare 6.1: Estimated fractional nacertainty in the background. 


\subsection{Fitting the Correlation Function}

When fitting the correlation function there are three areas of concern. The first is what function of the data should be minimized to extract the parameters, the second is what are the atatiatical uncertainties in the extracted parameters, and the third is how to estimate the probability that the theory used describee the data.

There are $25^{3}=15625$ bins in the histograms, wo the average number of counts per bin in small and therefore, the correlation function cannot be calculated by just calculating $R_{i j k} / B_{i j k}$ and then trying to ft a curve uning a loast squaren ft. Inotead, the Principle of Maximum Likelihood is used. In its most genoral form, one attempts to maximize the probability that the measured data came from the theory, by varying parametera in the theory[48]. The details of applying the principle to this problem are given in [1] and the main reaults will be summarized here. When applied to this experiment, one maximizes the probability that the real counts in a bin came from the measurement of a. number of counts equal to the background counts in that bin times the correlation function. Assuming that the real counts are Poisson distributed, and that the fluctuations in the predicted values (e.g., $D \cdot C_{i j k} \cdot B_{i j k}$ ) are negligible compared to the fluctuations in the real counte, this is

$$
\Phi=\prod_{i j k} \frac{\left(D \cdot C_{i j k} \cdot B_{i j k}\right)^{R_{i j h}}}{R_{i j k !}} e^{-D \cdot C_{i j k} \cdot D_{i j h}}
$$

where the product is restricted to bins where $B_{i j k} \neq 0$. Based on Eq. 6.59 and the arguments given in [1], the eatimated fluctuations in the background counts go $23 n^{3 / 4}$. So, to make the fluctuations in the background counts amall, all possible events are used in Eq. 6.57. When making the fits, it is easier computationally to minimize the quantity $F=-\ln \$$ rather than maximize the quantity $\$$. The correlation function is then fit by adjusting the parameters in $C_{i j k}$ (and $D$ ) to minimize $F$, the negative log of the likelihood function. The computer program used for the numerical minimization and estimates of the uncertainties was MINUIT[49].

The uncertainty estimates were made from contour plots of the log-likelihood function $(F)$, where the contour of intereat is that of the minimum value +0.5 . This contour would correspond to a $1 \sigma$ level if this were a $\chi^{2}$ fit. Note that the probability of all the parameters

\footnotetext{
'Recall the bintograme ueed 25 bine on exch of the three sides.
} 
being inside this contour is about $10 \%$ for the number of parameters present in our fits[50]. The uncertuinties given are taken from the tangent method, which uses the largest and smallest values inside the contour for a parameter, without regard to the values of the other parameters. This implies that the probability of this parameter's true value being within the uncertainty quoted is the ame a for a $1 \sigma$ interval, without regard to the values of the other parameters[50]. Some confidence contours and their corresponding uncertainties are given in Chapter 7.

There are two eatimators of the quality of the fit (once the fit has been made) used in this experiment. The firat is a reatricted $x^{2} /$ NDF and the second is the $x_{\text {inL }}^{3} / \mathrm{NDF}$, a defined in Eq. 6.61.

The $x^{2} /$ NDF is calculated in the usual manner, however only those bins predicted (based on $D \cdot C_{i j k} \cdot B_{i j h}$ ) to have more than five counts are used[48]. The background fuctuations, a calculated in Section 6.6, are not included in the uncertainty entimates. The resson for the second was simply that it was found that including the fluctuations did not change the value very much (which is good since one wants the background fluctuations to be small). The reason for the first in that the $\chi^{2}$ calculation asumes that the error distribution is Gaussian. Although the real spectrum is assumed to be Poisson distributed, for more than 5 counts per bin the two distributions are essentially identical. The distribution of the reatricted $\chi^{2}$ is that of a true $\chi^{2}$ with the number of degre ss of freedom in the range of $N$ to $N-L$ where $N$ is the number of bine, and $L$ is the $r . m b e r$ of parameters in the fit[48]. Note that the number of degrees of freedom reported here is always $N-L$, where $L=5$ for the two-radius parameter fits. This estimator has the virtue of allowing confidence levels to be estimated using standard methods, and one can verify the quality of the fit. This eatimator has the failing of not being sensitive to the entire region that is being fit. However, since the fit is made using the Principle of Maximum Likelihood, the fit is unbiased and, presumably, the quality of the fit is the same in any region.

The $x_{\text {Pul }}^{2} /$ NDF is derived from the Principle of Maximum Likclibood fitting method and is calcslated according to the prescription of [1]. The parameters were fit by minimizing the quantity $F=-\ln \$$. The related quantity, $x_{\text {Sul }}^{2}=2 F+$ (constant), has the additional property that it reduces to the usual $\chi^{2}$ in the limit of sufficient statistica, if 
the constant is chosen properly. With the proper constant the expression is

$$
\chi_{F M L}^{2}=-2 \ln \left\{\prod_{i j k} \frac{\left(D \cdot C_{i j k} \cdot B_{i j k}\right)^{R_{i j k}}}{R_{i j k} !} e^{-D \cdot C_{i j k} \cdot B_{i j k}}\right\}-\sum_{i j k} \ln \left\{2 \pi R_{i j k}\right\}
$$

where the product and the sum are restricted to those bins where $B_{i j k} \neq 0$. Although this estimator is unbiased even if the number of counts is small, it is difficult to determine exactly what the confidence levela are from standard methoda, since the distribution is not known[50]. This estimator has the virtue of being unbiased and the flaw not not allowing confidence levels to be calculated.

\subsection{Summary of the Fitting Procedure}

The procedure for fitting the correlation function is then: a functional form for the $C_{i j k}$ is assumed, and the free parameters (in Eq. 3.14 these are $\lambda, R_{\|,} R_{\perp}$ and $\tau$ ) are set to values corresponding to typical nuclear sizes. Equations 6.40 , and 6.50 are used to calculate weights and Eq. 6.50 is iterated until stable to further iteration. The $R_{i j k}$ and $B_{i j k}$ are calculated from Eqs. 6.56 and 6.57 using the weights. The parameters in the correlation function are adjusted to maximize agreement in Eq. 6.55 to give new correlation function parameters. This correlation function is used to calculate new weights and the procedure is repeated unt:il the correlation function parameters are stable to further iteration. Generally less than five iterations are required. This procedure has been checked using Monte Carlo data with a known correlation function (using Eq. 3.14) and has been verified to converge to th: input parameter values. The results from the Monte Carlo tests are shown in Section 7.2. 


\section{Chapter 7}

\section{Results from the Correlation}

\section{Function Fits}

\subsection{Presentation of the Data}

The data (both the real counts and the background, or reference sample, counts) were histogrammed as a function of $q_{\|}, q_{1}$ and $q_{0}$. The fits were made using these three dimensional histograms. Displaying the fits in the obvious fashion of fixing, for example, $q_{\eta}, q_{1}$ and showing the data 25 a function of $q_{0}$ has two features. The first is that thare would be $25^{2}=625$ such displays, the second is that most of the bins in any given display would be empty, both from limited statistics and from the acceptance limits of the spectrometer in $\mathbf{Q}$ space. $^{1}$

Therefore, averages are made over (again, for example) the $q_{\|}-q_{\perp}$ plane, at a given value of $q_{0}$, to display averages of the qrantities as a function of $q_{0}$. By using each of the three variables $\left(q_{\|}, q_{\perp}\right.$ and $\left.q_{0}\right)$ in turn, three displays are made showing the data and the fitted correlation function projected onto each of the coordinate axes. Note that these are averages weighted by the spectrometer's acceptance - the projections of the data and the fitted correlation function are both distorted by the acceptance.

The definition of the data points in the projection $\left(C_{k}\right)$ is

$$
\mathcal{C}_{k}=\frac{\sum_{i j} R_{i j k}}{D \cdot \sum_{i j} B_{i j k}}
$$

\footnotetext{
${ }^{1}$ There is, in addition, the physical constraint that for particle pairs, $q_{1}^{2}+q_{\perp}^{2} \geq q_{0}^{2}$.
} 
the uncertainty in the data points is estimated as

$$
\left(\Delta \mathcal{C}_{k}\right)^{2}=\mathcal{C}_{k}^{2}\left\{\frac{1}{\sum_{i j} R_{i j k}}+\frac{\sum_{i j}\left(\Delta B_{i j k}\right)^{2}}{\sum_{i j}\left(B_{i j k}\right)^{2}}\right\}
$$

and the fitted curve is given by

$$
\left\langle C_{k}\right\rangle=\frac{\sum_{i j} \sigma_{i j k} \cdot B_{i j k}}{\sum_{i j} B_{i j k}}
$$

where $D$ is the over-all nornalization constant (which is fit, sen Eq. 6.35), $R_{i j k}$ is the real counts per bin, $B_{i j h}$ is the seckground (or reforence ample) counte per bin and $\Delta B_{i j h}$ is the entimated uncrertalaty in the background conats per bin (men Eq. 6.56, 6.57 and 6.59). In Equations 7.1, 7.2 and 7.3, the lat index ( $k$ ) was kept and the first and recond indices ( $i$ and $j$ ) were averaged over. By keeping either the first, or the second, index (e.g., $i$ or $j$ ) and averaging over the remaining indices, a total of three rets of similar equations can be preduced, each set of equations correspriading to the averages as a function of one of $90, q \perp$ and $q 1$. Note that if $R_{i j k}=D \cdot C_{i j k} \cdot B_{i j k}$ the curves will be cloce to the data points. From Eq. 7.3 one can are how the averages are weighted by the spectrometer's acceptance.

\subsection{Fits to Monte Carlo Data}

Section 6.9 referred to a teat of the correlation function fitting routines using Monte Carlo data with a known correlation function. The data for this test were generated so that the correlation function was the same as that predicted by the theory, Eq. 3.14, with $\lambda=1$, $R_{\|}=3, R_{\perp}=4$ and $r=2$. The Moate Cario pions were then processed by nimulatinf, the spectrometer a used in the $0^{\circ} \mathrm{Nb}$ set up with $\left|\vec{p}_{\text {rojoj }}\right|>30 \mathrm{MeV} / \mathrm{c}$ (see Table 4.2). The Monte Carlo program is deacribed in Appendix A. The data output from the Moats Carlo simulation were paned to the neual data analyais strenm with the same geometrici'l cutu as uned in the real data analyas. The only difference was that the pulse-height and time-of-light cuts in the sciatillation counters were not uned siace purticle contamination was not simulated. This procedure gives a close approximation to the spectrometer's momentum limits and two track efficiency efrects.

Table 7.1 show the parameters extracted from tine fit to the Monte Carlo data. The confidence level for this fit (based on the $\chi^{2} / \mathrm{NDF}$, wee Section 6.8) is $35 \%$. In Figures 7.1, 


\begin{tabular}{|c|c|c|}
\hline Parameter & Input value & Fitted value \\
\hline \hline$R_{\perp}(\mathrm{fm})$ & 4.0 & $3.8 \pm 0.2$ \\
$R_{\|}(\mathrm{fm})$ & 3.0 & $3.2 \pm 0.3$ \\
$\tau(\mathrm{fm} / \mathrm{c})$ & 2.0 & $2.2_{-0.4}^{+0.6}$ \\
$\lambda$ & 1.0 & $0.98 \pm 0.05$ \\
$\chi^{2} / \mathrm{NDF}$ & - & $595 / 600$ \\
$\chi_{\text {FuL }}^{2} / \mathrm{NDF}$ & - & $2646 / 2359$ \\
Events & - & 14000 \\
\hline
\end{tabular}

Table 7.1: Parameters extracted from Monte Carlo simulated events. Only atatiatical uncertainties are shown.

7.2 and 7.3 the projections for this fit are shown. In Figures 7.4, 7.5 and 7.6 the corresponding confidence coutours are shown.

In Figures 7.1, 7.2 and 7.3 some effects of the spectrometer's acceptance can be seen: First, the intercept \&s $q \rightarrow 0$ is not $C_{2}=1+\lambda$ w one might expect from Eq. 3.14. This is because the average at, for example, $q_{0}=0$ includes bins where $q_{\perp}$ and $q_{\|}$are not zero, and $C_{2}<1+\lambda$. Similar arguments apply for the other projections. Second, the shapes of the data and the fitted curves do not follow Eq. 3.14, the most obvious being ior qn in Fig. 7.3. However, the projections for both the data and the fitted correlation function have the same shape indicating that this is an acceptance effect. See Equation 7.3 to see how the fitter correlation function is weighted by the acceptance.

One can see that a spectrometer with a limited angular acceptance couples the de. termination of the radius parameter parallel to the direction of nbservation and the lifetime parameter. The cause can be visualized by imagining two pion momentum vectors constrained to be within some small angular range. The variation of angle (at a fixed energy) gives momentum differences perpendicular to the average $\vec{p}$ of about gporpendicular $\approx|\vec{p}| 2 \operatorname{con}\left(\frac{1}{2}\right)$, which is large. Therefore, the momentum difference perpendicular to the pion momentum is decoupled from the energy difference, and the lifetime parameter is decoupled from the corresponding radius parameter. The variation of angle (at a fixed energy) gives momentum difference parallel to the average $\vec{p}$ of about $q_{\text {pardllel }} \approx|\vec{p}| 2 \sin \left(\frac{4}{2}\right)$, which is small, whereas varying $|\vec{p}|$ gives large variations in the 


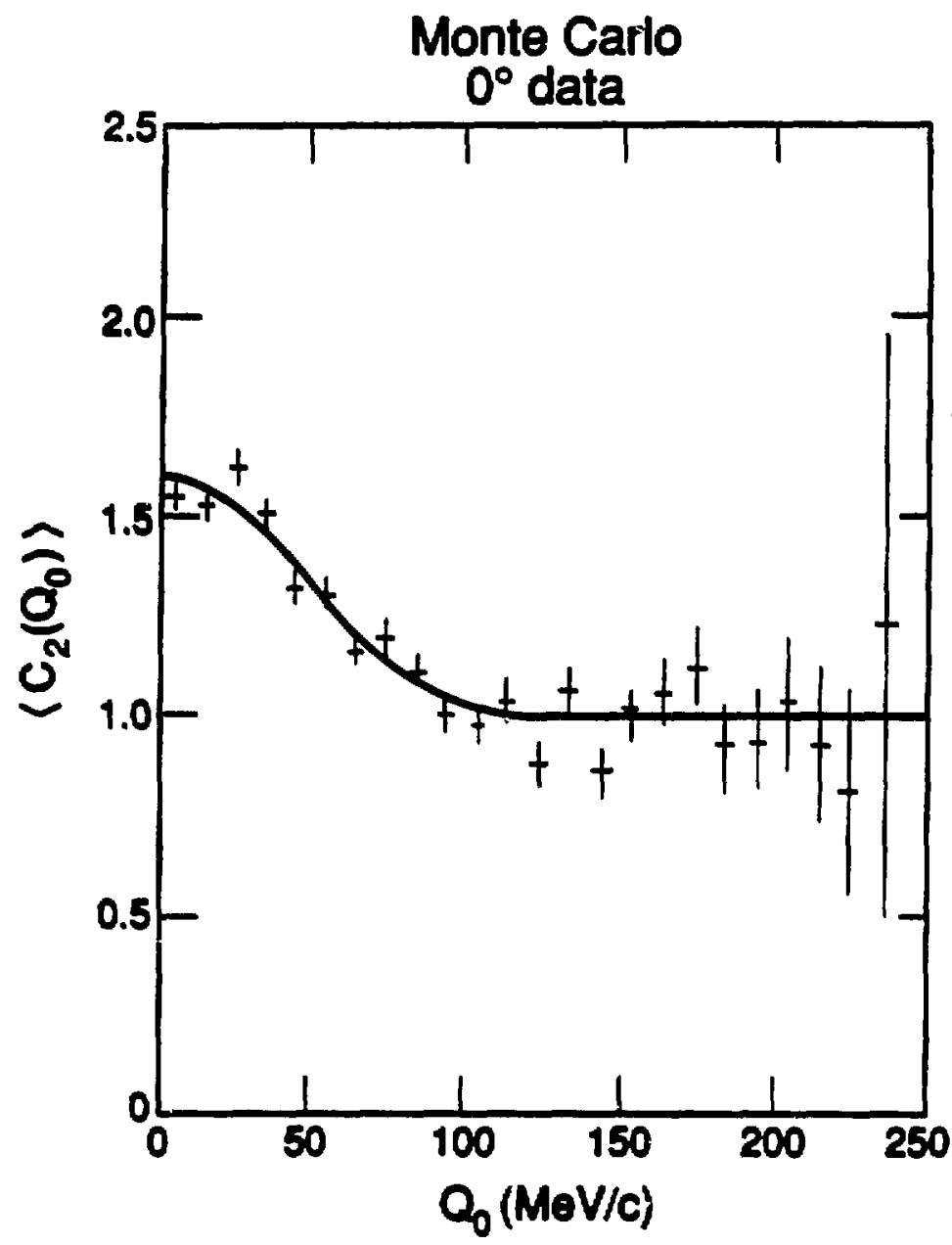

Figure 7.1: $\left\{C\left(C_{0}\right)\right\rangle$ for the Monte Carlo data. Only statiotical uncertainties are shown. 


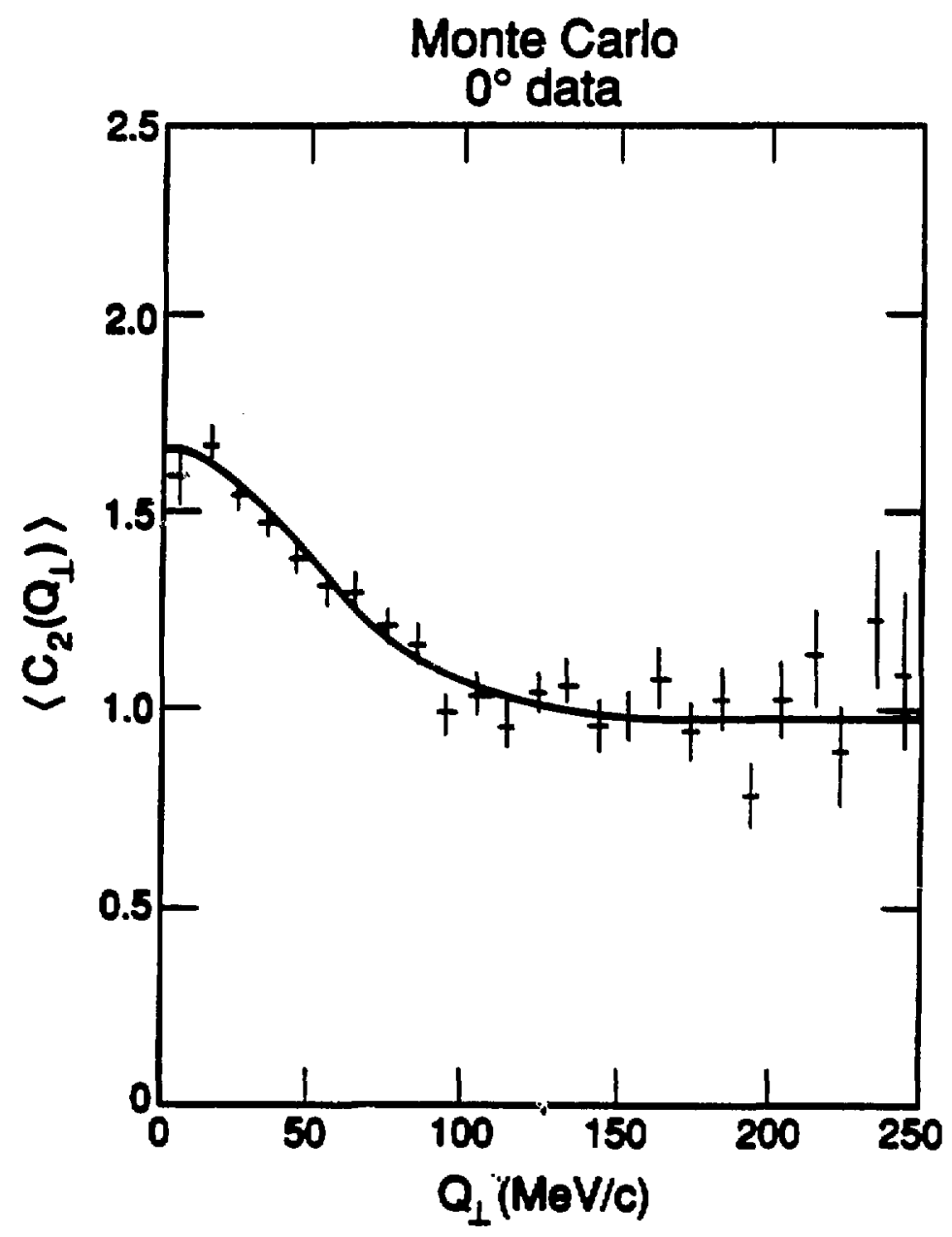

Figure 7.2: $\left\{C\left(\varphi_{1}\right)\right\}$ for the Moate Carlo data. Only statistical uncertainties are shown. 


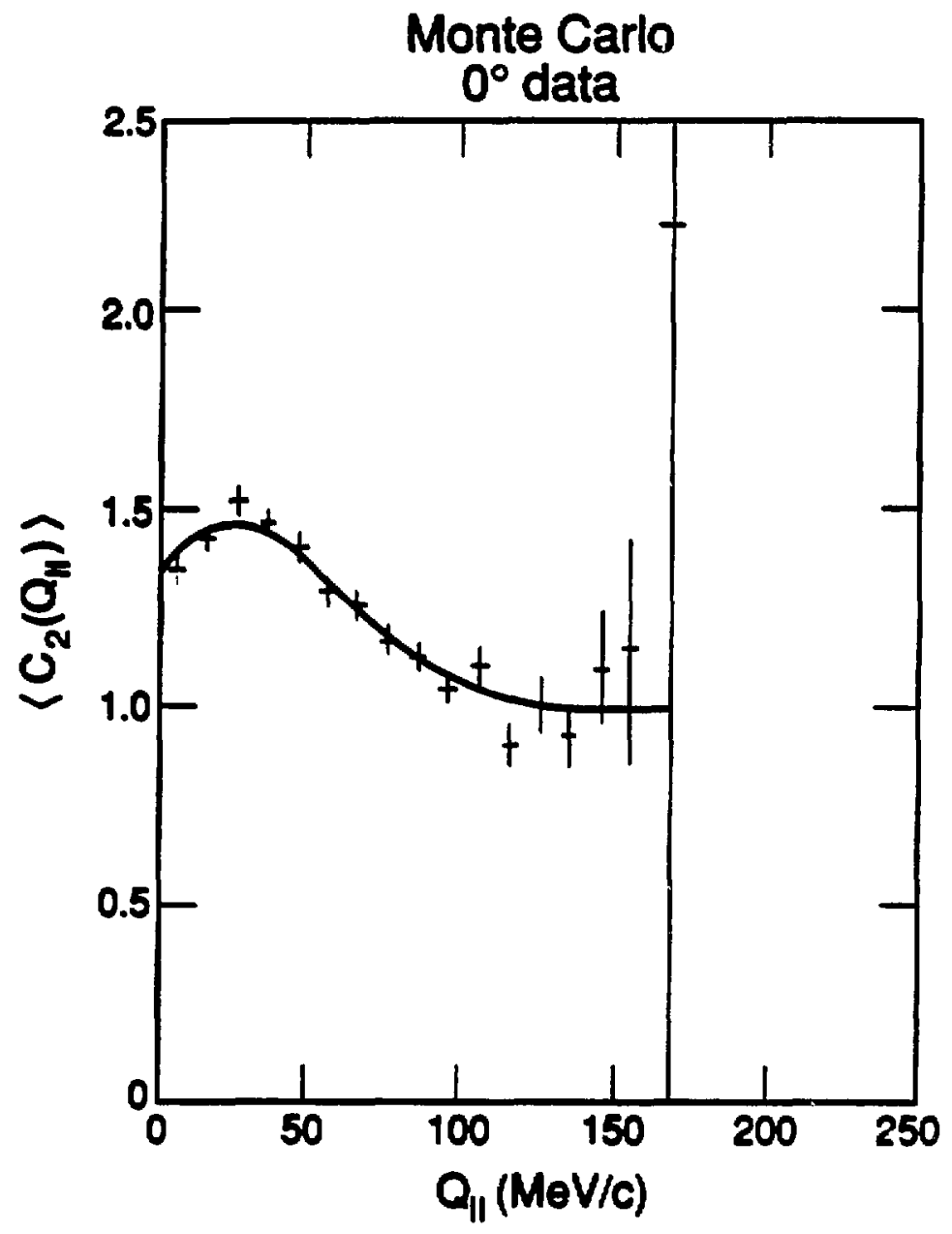

Figure 7.3: $(C($ q1 $))$ for the Monte Carlo data. Only atatistical uncertainties are shown. 


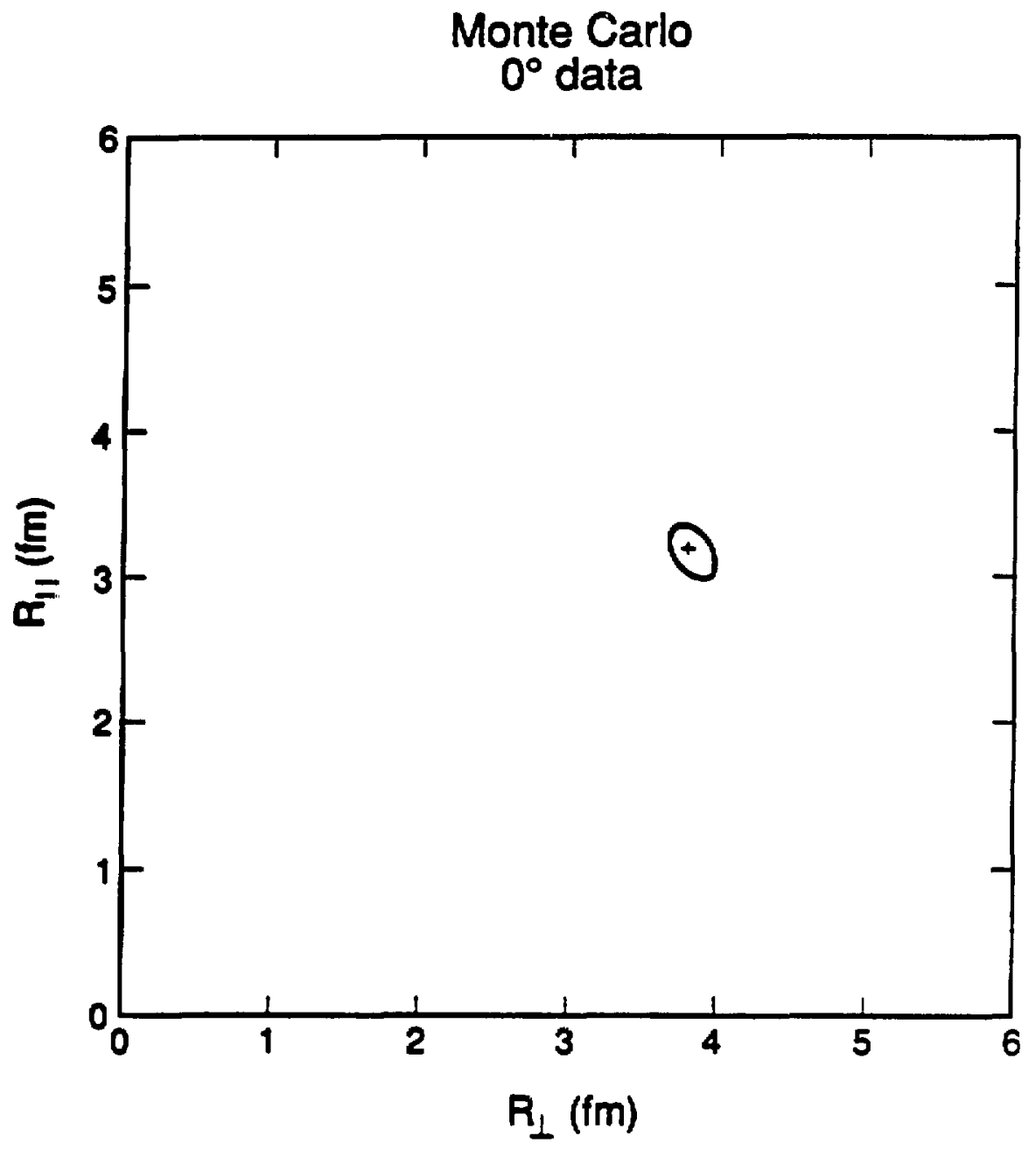

Figure 7.4: The 1e confider ce contour for the Monte Carlo data $-R_{\|}$ve. $R_{\perp}$ plane. 


\section{Monte Carlo $0^{\circ}$ data}

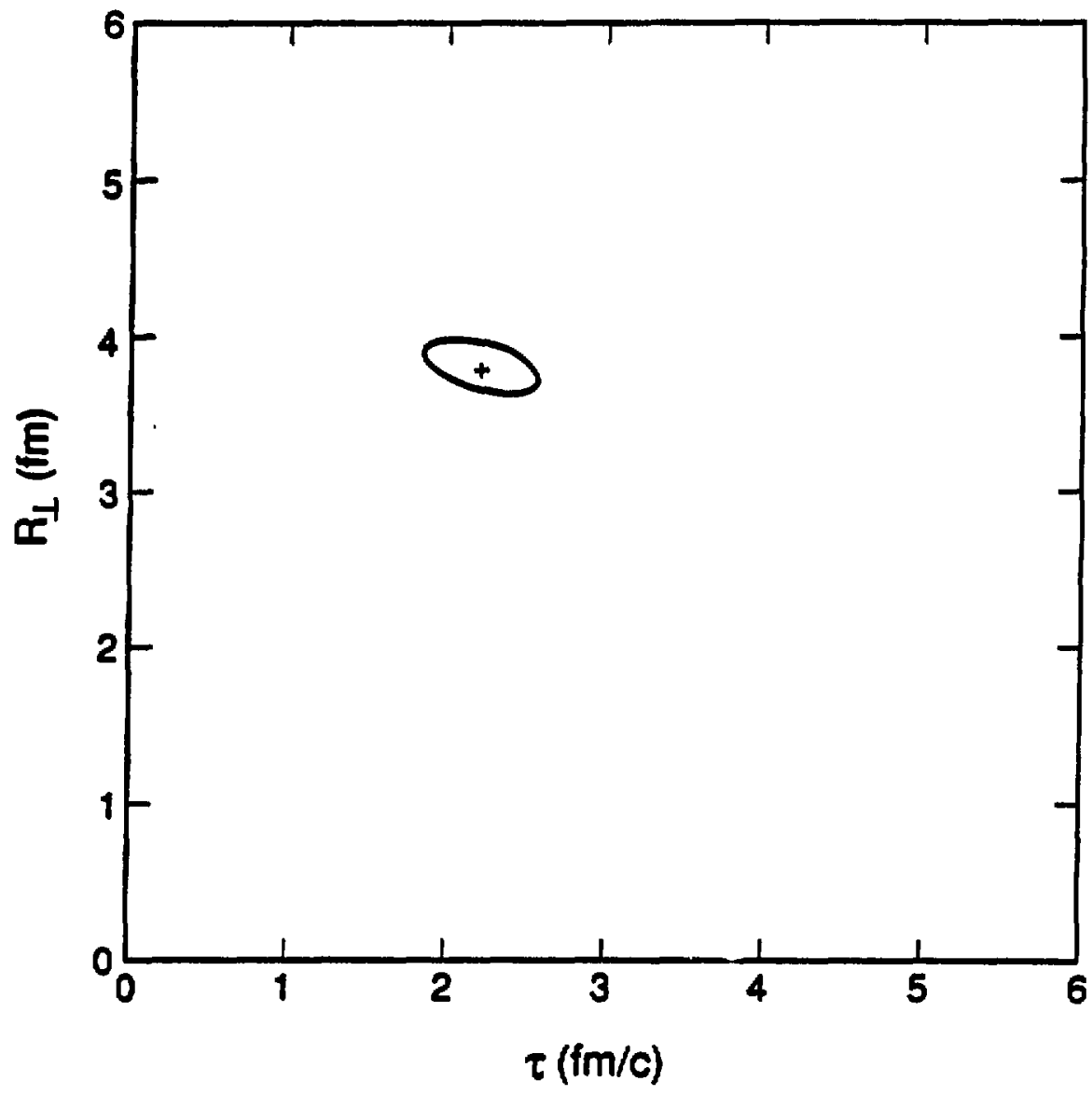

Figure 7.5: The 10 confidence contour for the Monte Carlo data $-R_{\downarrow}$ vs. $T$ plans. 


\section{Monte Carlo \\ $0^{\circ}$ data}

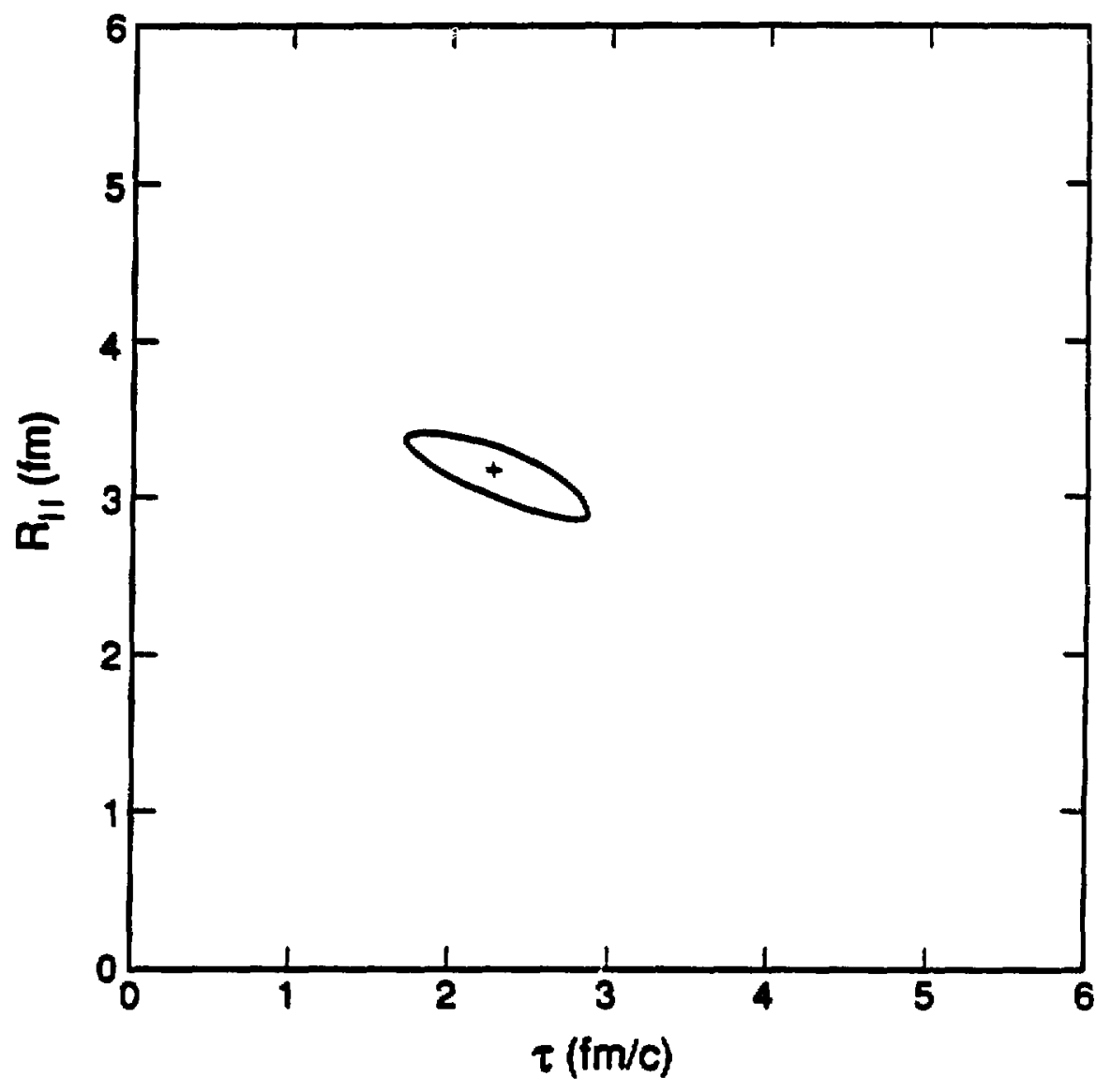

Figure 7.6: The 10 confidence coatour for the Moate Carlo data $-R_{\|}$va. $r$ plane. 
momentum difference parallel to the average momentum while varying the energy difference as well. Therefore, the momentum difference parallel to the direction of observation will be coupled to the energy difference and one cannot easily separate the lifetime and the corresponding radius parameter.

In the confidence contour plots a coupling between two variables causes the principle axes of the confidence contour ellipee to not be parallel to the coordinate axes. Instead, the axes are parallal to whatever combinations of the variables are independent. In the confideace contour plota for the Monte Carlo data one can see the greater cuupling between $R_{\|}$and $r$, than between $R_{\perp}$ and $r$. Note that the Moate Carlo was a simulation of the $0^{*}$ spectromater where this is expected since the pion momenta are constrained to be to within an angular range clowe to the beam axis.

\subsection{Projections for the Data}

Figures $7.7,7.8$ and 7.9 show the projections for the $0^{\circ} \mathrm{Nb} \pi^{-}$data sample with $\left|\vec{p}_{\text {proj }}\right|>$ $30 \mathrm{MeV} / \mathrm{c}$. This data sumple wa chonea for display since it is the largest data sample and, correspondingly, has the smallest statistical uncertainty in the data points. For this reason any systematic effects will be the most anparent in this data set. These plots do not show any systematic differences between the data and the corselation function used to fit the data. Note the similaritie between the $0^{\circ}$ data and the Monte Carlo, which wa a simulation of the $0^{*}$ data. For comparion, the projections from the $45^{\circ} \mathrm{Nb}$ uncut data sample (this data sample is uncut in the senee that no cuts have been imposed that would remove correctly reconstructed pions, see Section 7.7) are shown in Figures 7.10, 7.11 and 7.12. This is a slightly smalles data set, but again, no systematic differences between the data and the fitted correlation function can be meen. The parameters deduced from these fits are listed in Tables 7.5 and 7.7 (which appear later in Section 7.7).

The projections, of cours, reduce the amount of data output (from $25^{3}=15625$ bins to $3 \times 25=75$ bins), 0 some information is lost in making the projections. This means that there are some poaible aystematic effects that could be manked in the projections. For example, bini along the line given by $q_{0}=q_{\|}=q_{\perp}$ could be high (or low), and this would not show in the projections. However, calculations of tine confidence levels from the $\chi^{2}$ /NDF (see Section 6.8) have verified that the confidence levels are acceptable. 


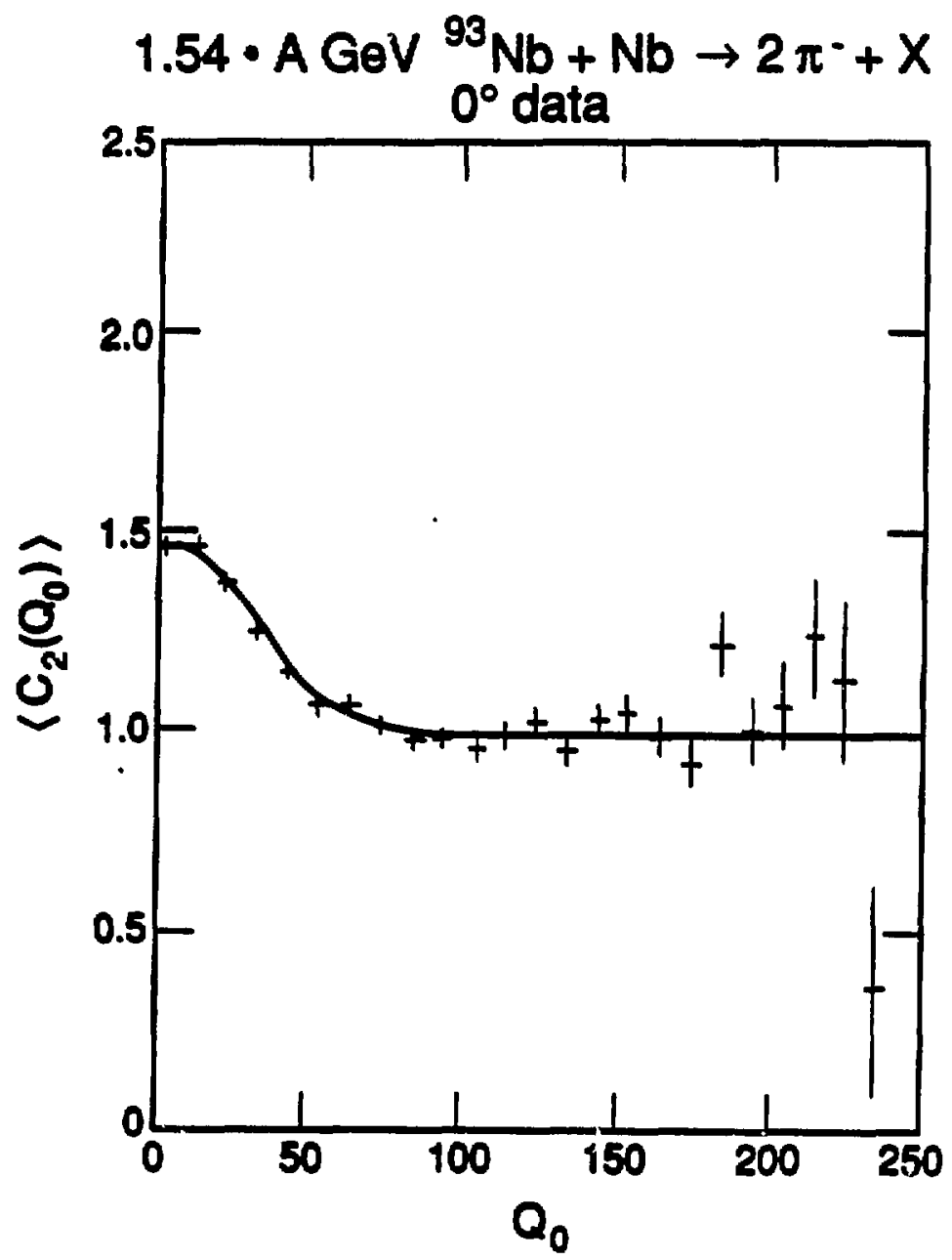

Figure 7.7: $(C(\varphi 0))$ for the $0^{\circ}$ No least cut data. Only atatiatical üncertaintied are shown. 


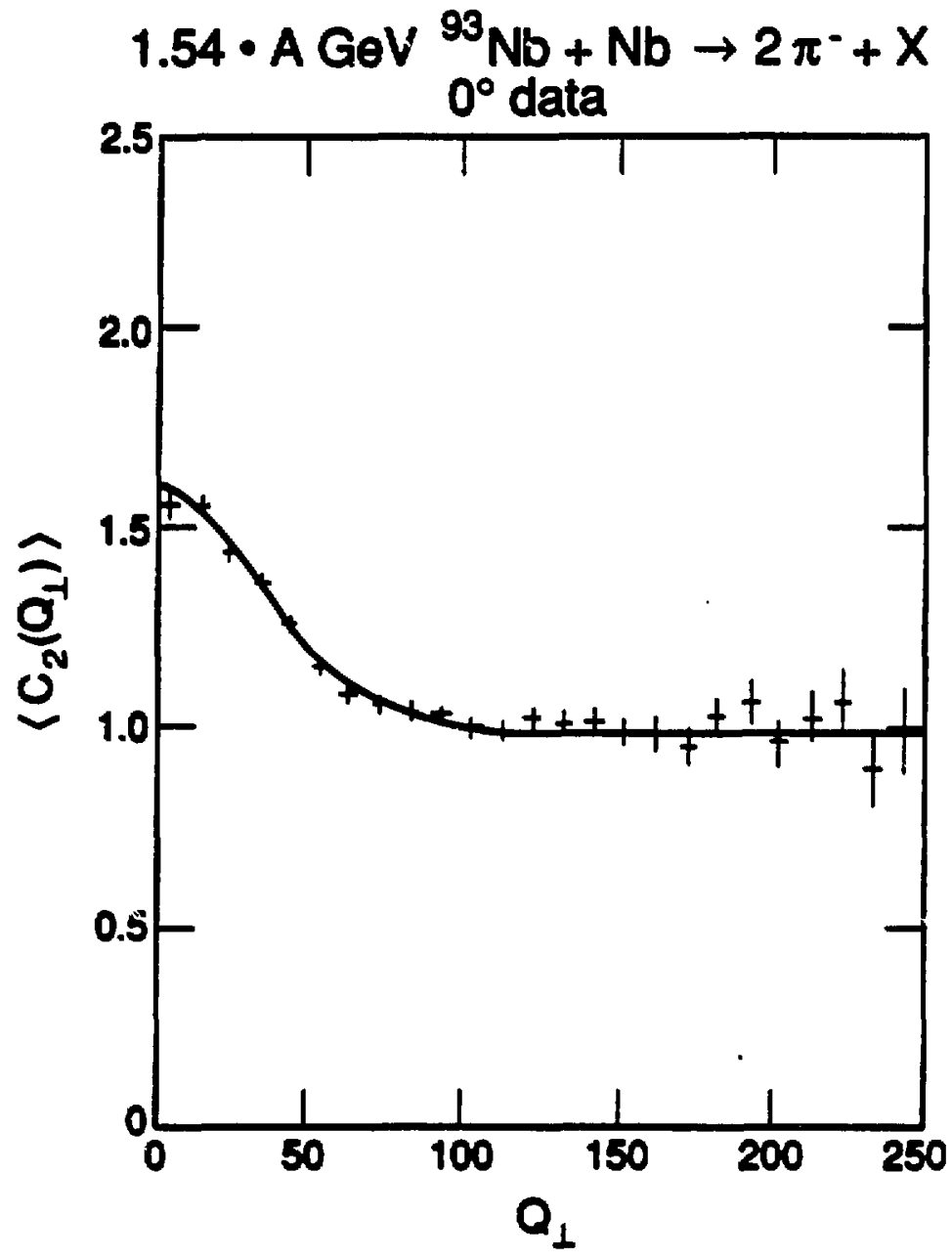

Figure 7.8: $\left(C\left(q_{1}\right)\right)$ for the 7 No least cut dat2. Only statistical uncertaintius are shown. 


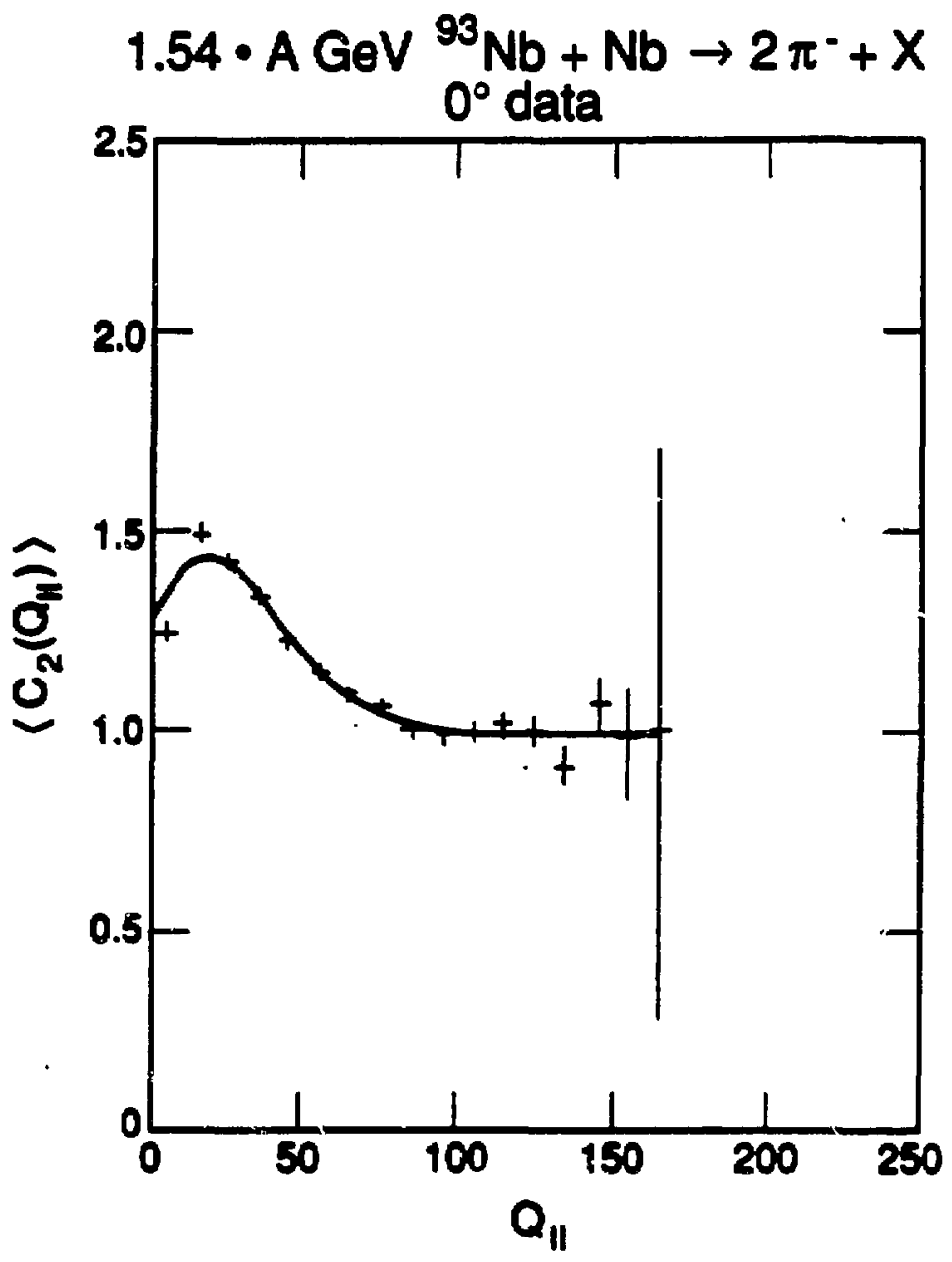

Figum 7.9: $(C(n)))$ for the $0^{\circ} \mathrm{Nb}$ last cut data. Only statistical uncertuinties are shown. 


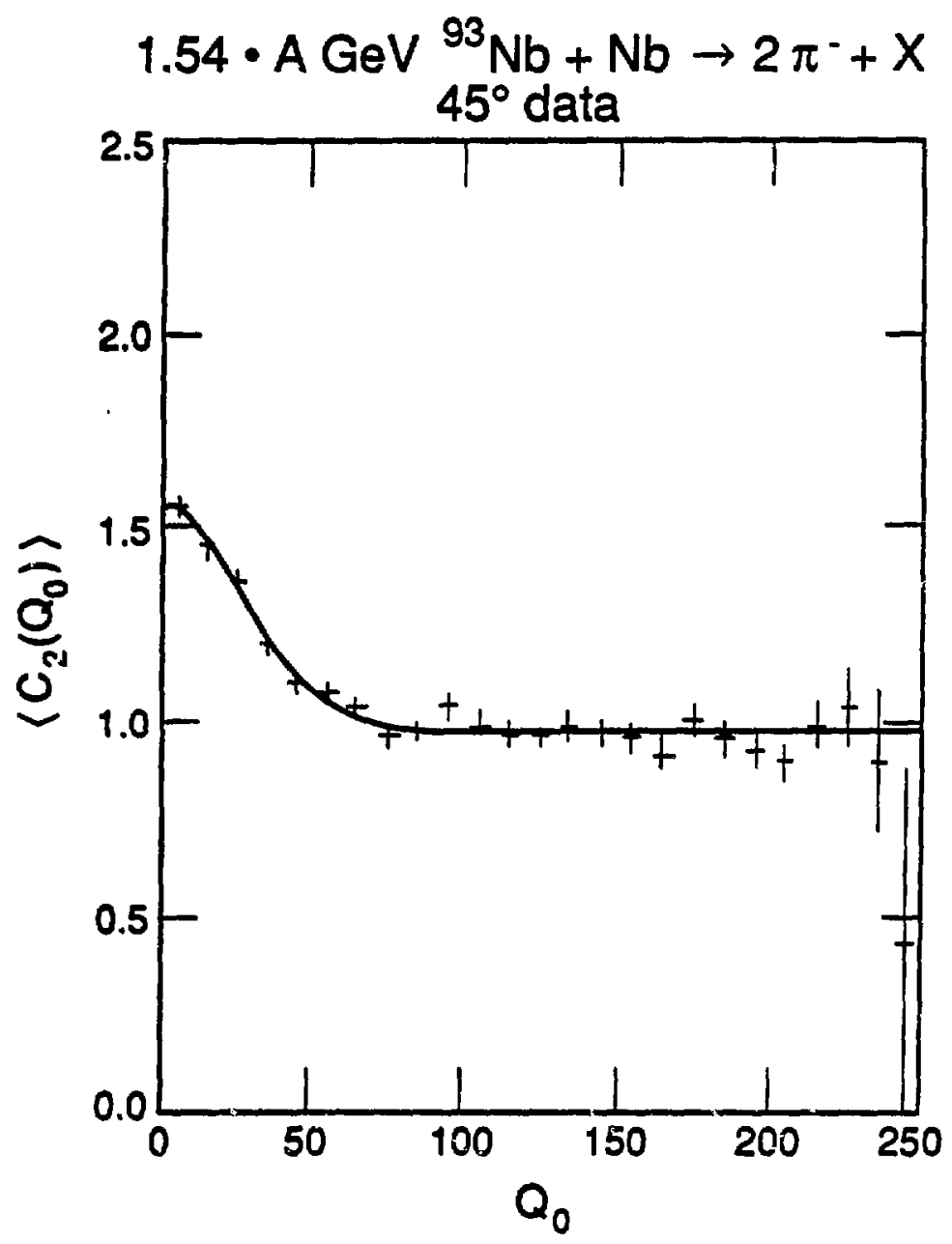

Figure 7,10: $\langle C(90)\rangle$ for the $45^{\circ} \mathrm{Nb}$ uncut datz. Only statistical uncertainties are shown. 

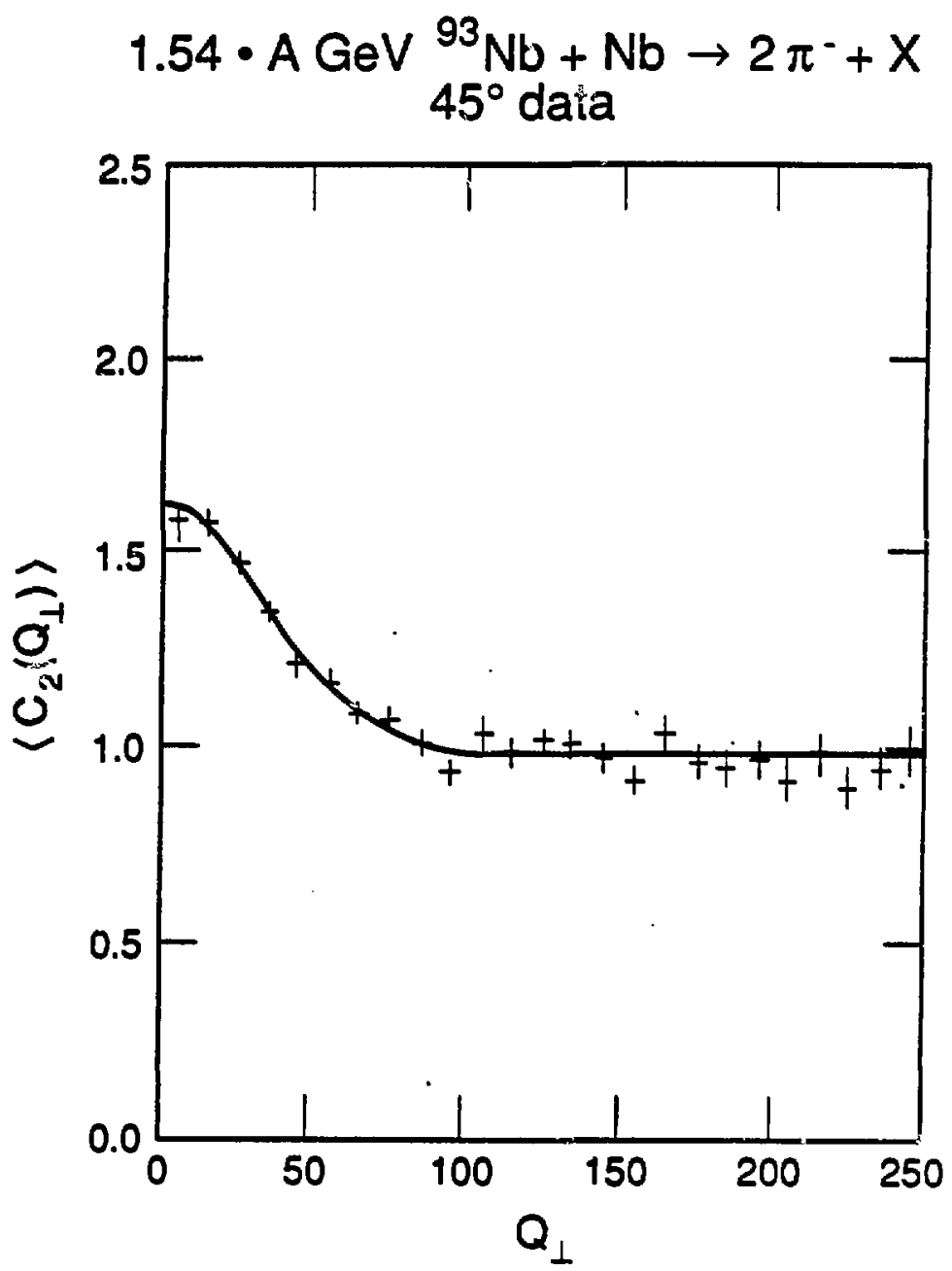

Figure 7.11: $\left(C\left(q_{\perp}\right)\right)$ for tie $45^{\circ} \mathrm{Nb}$ uncut data. Only statistical uncertainties are shown. 




Figure 7.12: $\{C($ s $)\rangle$ for the $45^{\circ} \mathrm{Nb}$ uncut data. Only statiatical uncertanties se shown. 


\subsection{Confidence Contours for the Data}

Figures $7.13,7.14$ and 7.15 show confidence contours for fits to the $0^{\circ} \mathrm{Nb} \pi^{-}$data sample with $\left|\vec{p}_{\text {proj }}\right|>100 \mathrm{MeV} / \mathrm{c}$, and Figures 7.16, 7.17 and 7.18 show confidence contours for the $45^{\circ} \mathrm{Nb}$ data with $\left|\vec{P}_{\mathrm{cm}}\right|>150 \mathrm{MeV} / \mathrm{C}$. The shapes of the contours are typical of the contours for all of the data sats, although for the data wets with fewer events the error contours can interaect zaro. Figure 7.19 shows the contour for one auch case. The data in this plot are for the $0^{\circ}$ Ar data ample with $\left|\vec{F}_{\text {proj }}\right|>50 \mathrm{MeV} / \mathrm{c}$; however, the appearance is typical for an error contour interencting zero. Note that for the $0^{*} \mathrm{Nb}$ dat,$R_{\|}$is more correlated with $r$ than $R_{\perp}$ is. For the $45^{\circ} \mathrm{Nb}$ data the situation is reversed, and the independence of $R_{\|}$and $r$ is clearer than for $R_{\perp}$ and $\tau$ in the $0^{\circ}$ case. The va'ues of the parameters for thewe fits, and the uncertaintien, are given in Tables 7.6, 7.8 (for Nb) and 7.7 (for Ar).

\subsection{Study of the Coulomb Corrections}

The Coulomb correction between the pions and the nuclear matter, and the correction between the two pions in the event (the corresponding correction is called the Gamow correction, although it is a Coulomb interaction), were discused in Section 6.7. In principle one has to solve the $\mathrm{n}$-body interaction problem to determine the corrections accurately. To determine if the asumption that the interactions can be separated is acceptable, a study was made using about $1 / 3$ of the $0^{\circ}$ Fe data (with $\left|\vec{p}_{\text {proj }}\right|>50 \mathrm{MeV} / \mathrm{c}$ ), where fits were made with all possible combinations of the two corrections being made or not. The parameters extracted from the fits are given in Table 7.2. Note that the $\left|\vec{p}_{\text {proj }}\right|>50 \mathrm{MeV} / \mathrm{c}$ cut is made after the corrections so that the number of events, hence the NDF, depends somewhat on the corrections applied.

Examining Table 7.2 show that the size of a correction does not depend on if the other correction is made or not. All confidence levels are in the range $10 \%$ to $15 \%$ (based on the $\chi^{2} / N D F$ ), so the quality of the fit does not depend on if a correction is made or not. Based on this test it is concluded that the corrections may be calculated separately, as was done. The motivation for the cut at $50 \mathrm{MeV} / \mathrm{C}$ in the $0^{\circ}$ data was a compromise between the data sample size and the dexire to have small Coulomb corrections, which 




Figure 7.13: The 10 confidence contour for the $0^{\circ} \mathrm{Nb}$ cut data $-R_{||}$va. $R_{\perp}$ plane. 


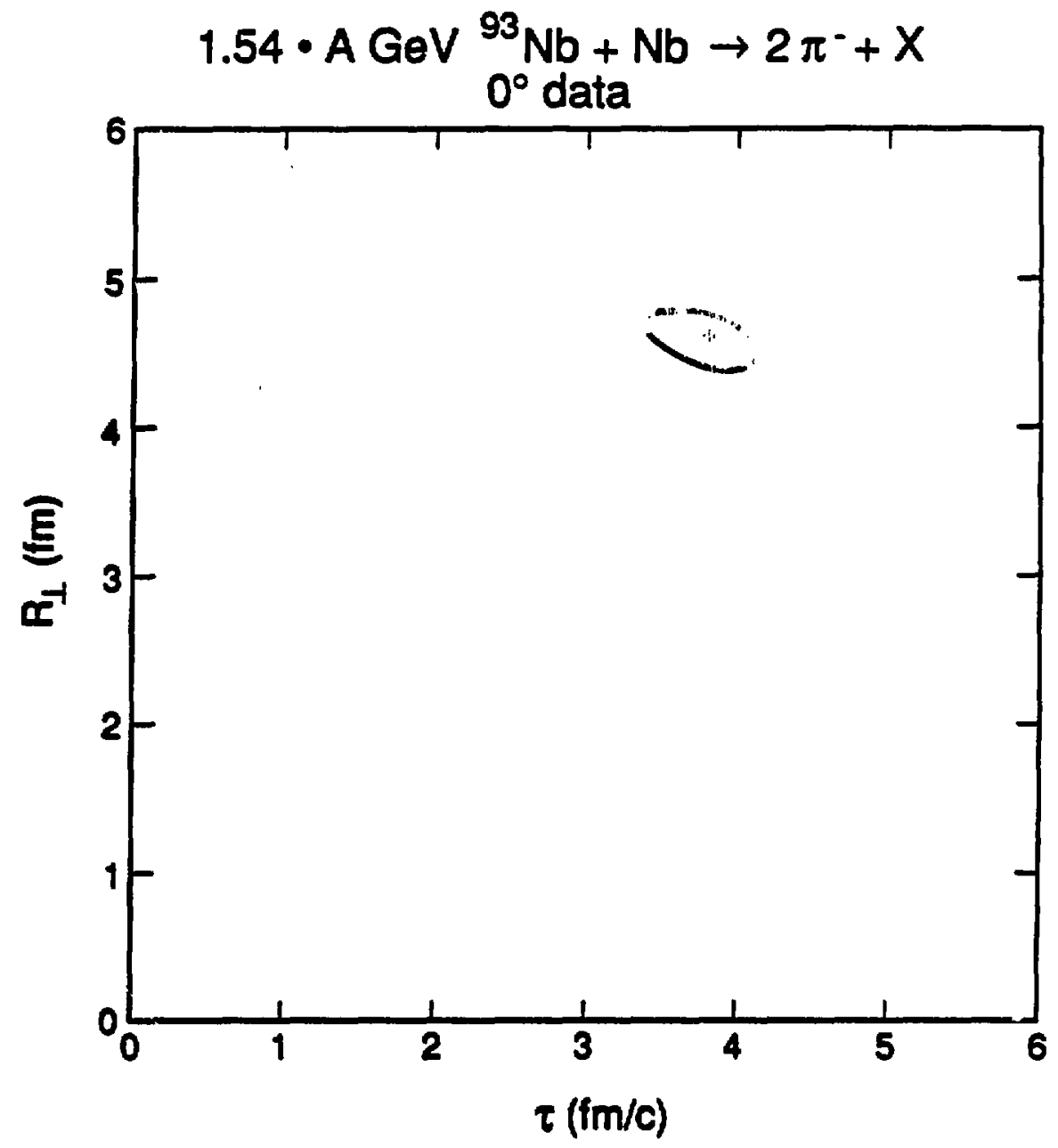

Figure 7.14: The 10 coafidence coatour for the $0^{\circ} \mathrm{Nb}$ cut data $-R_{\perp}$ vs. $\tau$ plane. 


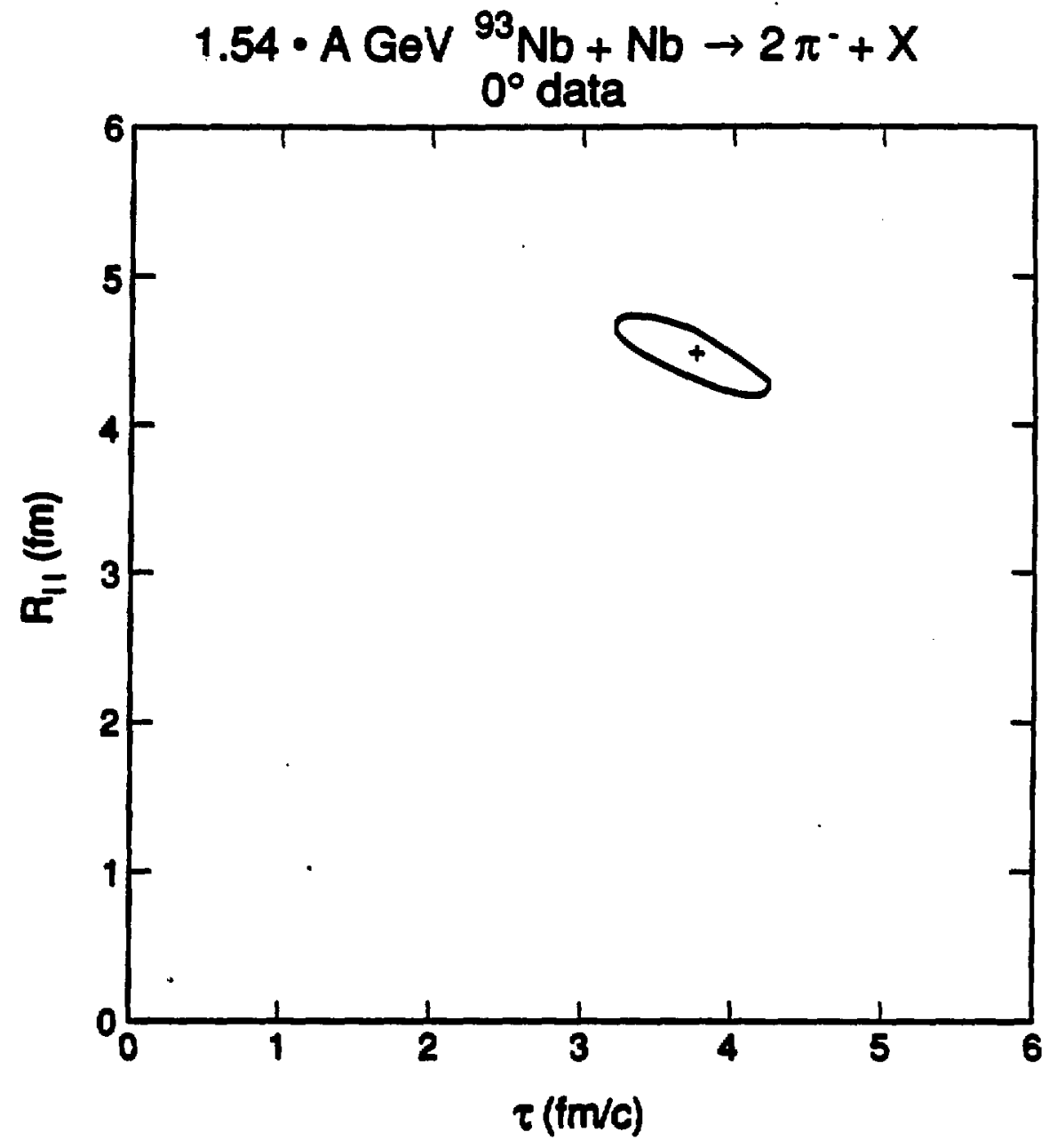

Figure 7.15: The $1 \sigma$ confidence contour for the $0^{\circ} \mathrm{Nb}$ cut date $-R_{\|}$vs. $T$ plene. 


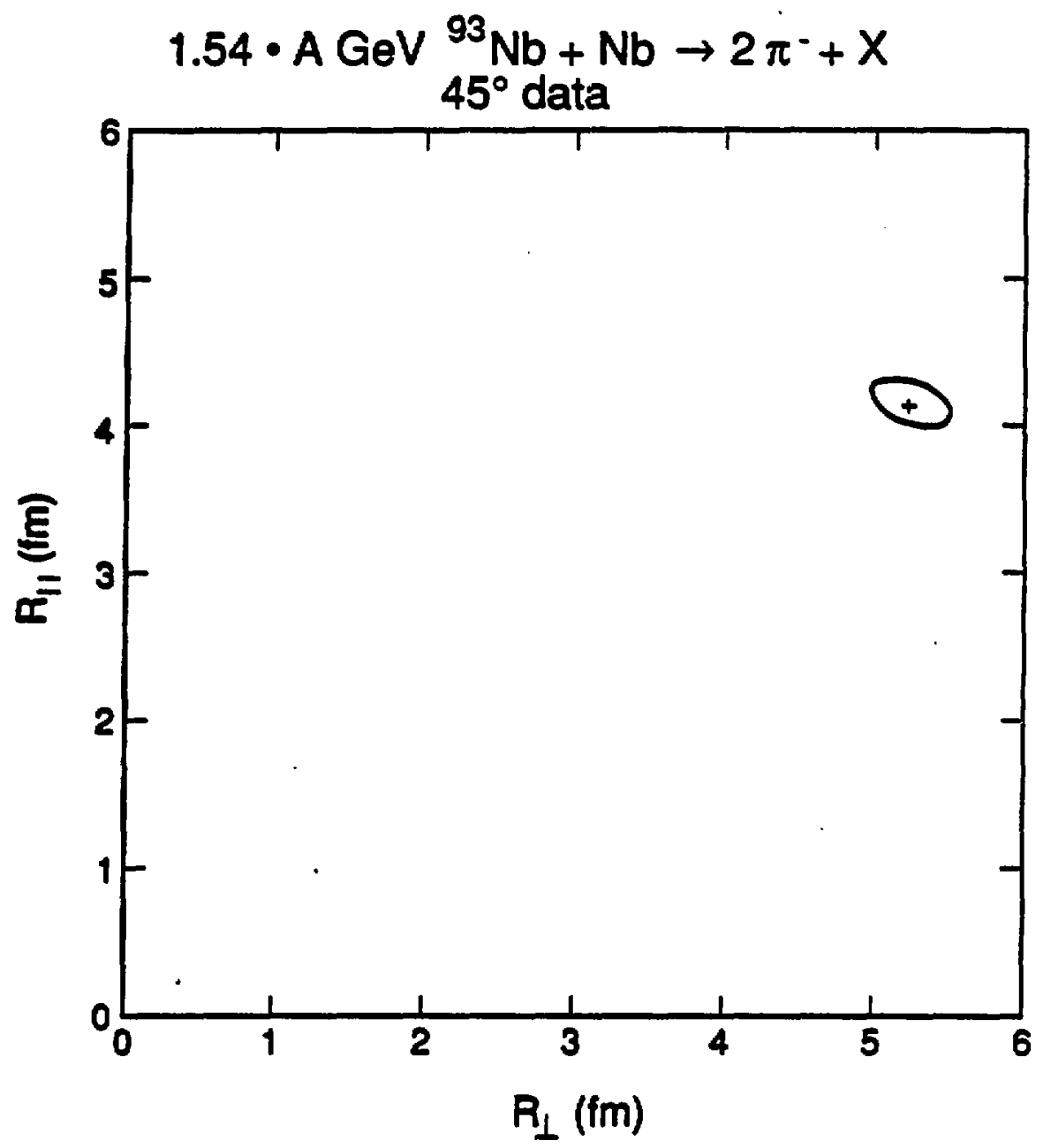

Figure 7.16: The $1 \sigma$ confidence coatour for the $45^{\circ} \mathrm{Nb}$ cut data $-R_{\|}$vs. $R_{\perp}$ plane. 


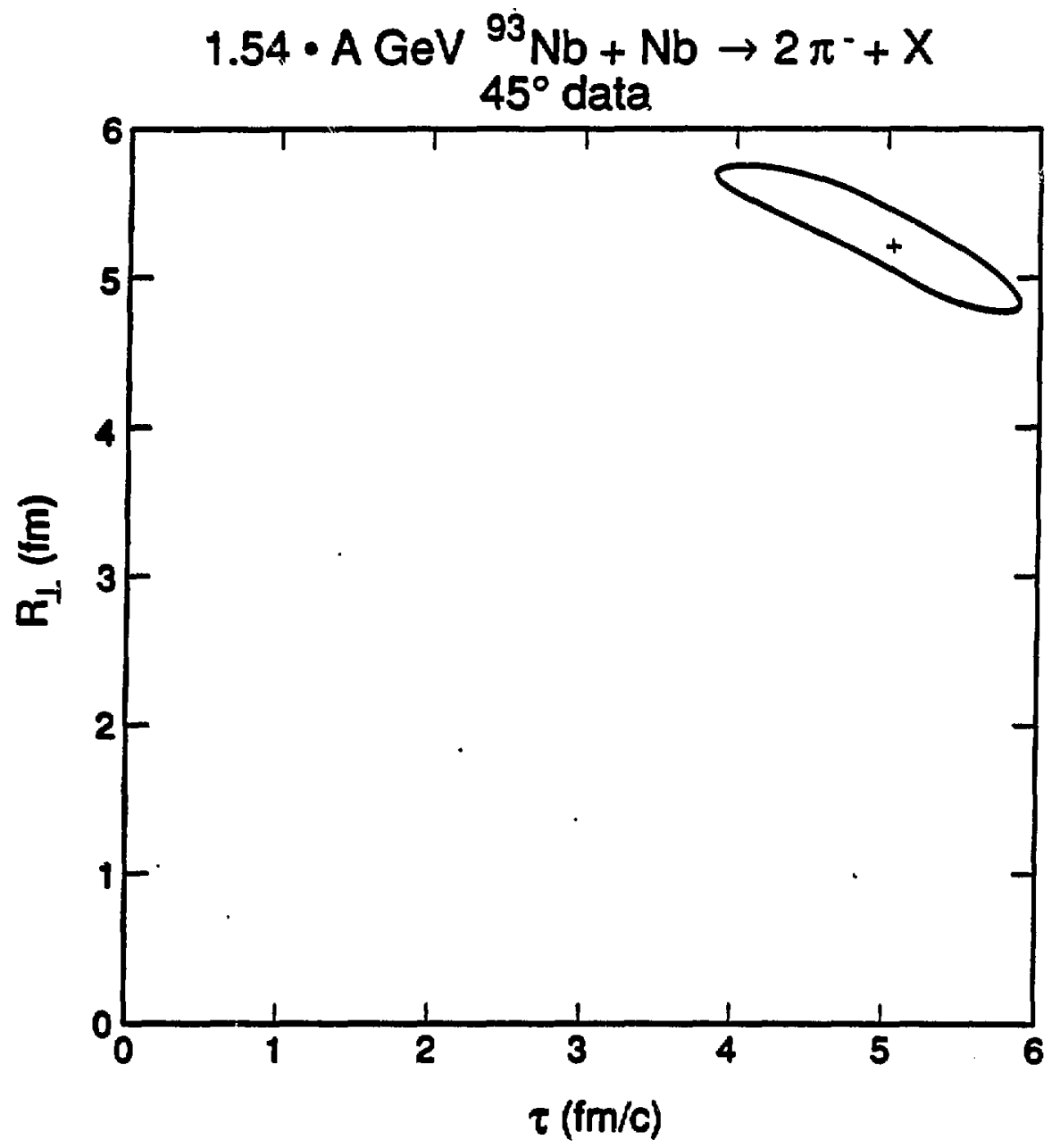

Figure 7.17: The 10 confideace contour for the $45^{\circ} \mathrm{Nb}$ cut data $-R_{\perp}$ va. $\tau$ plane. 


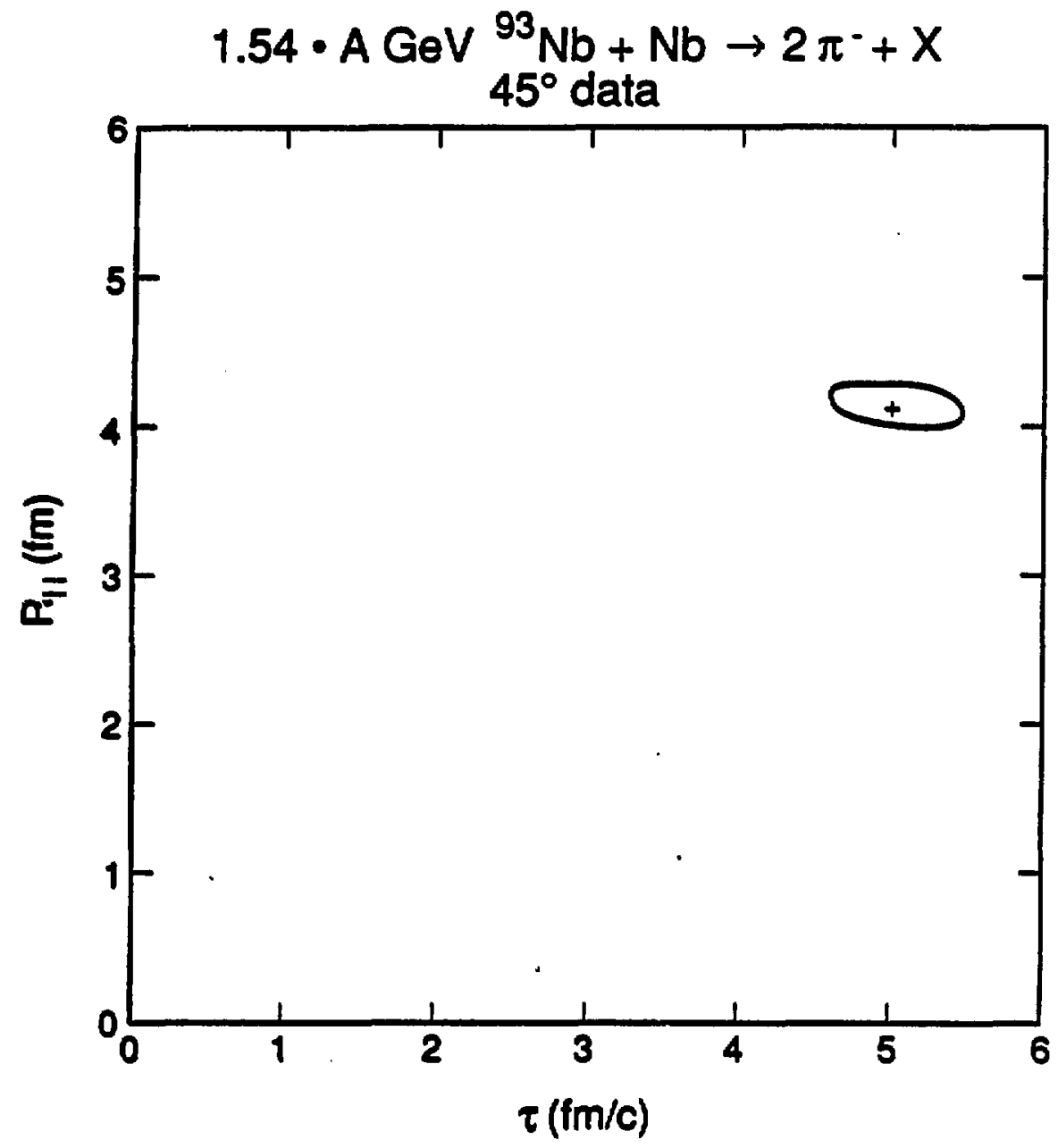

Figure 7.18: The $1 \sigma$ confidence contour for the $45^{\circ} \mathrm{Nb}$ cut data $-R_{\|}$vs. I plane. 


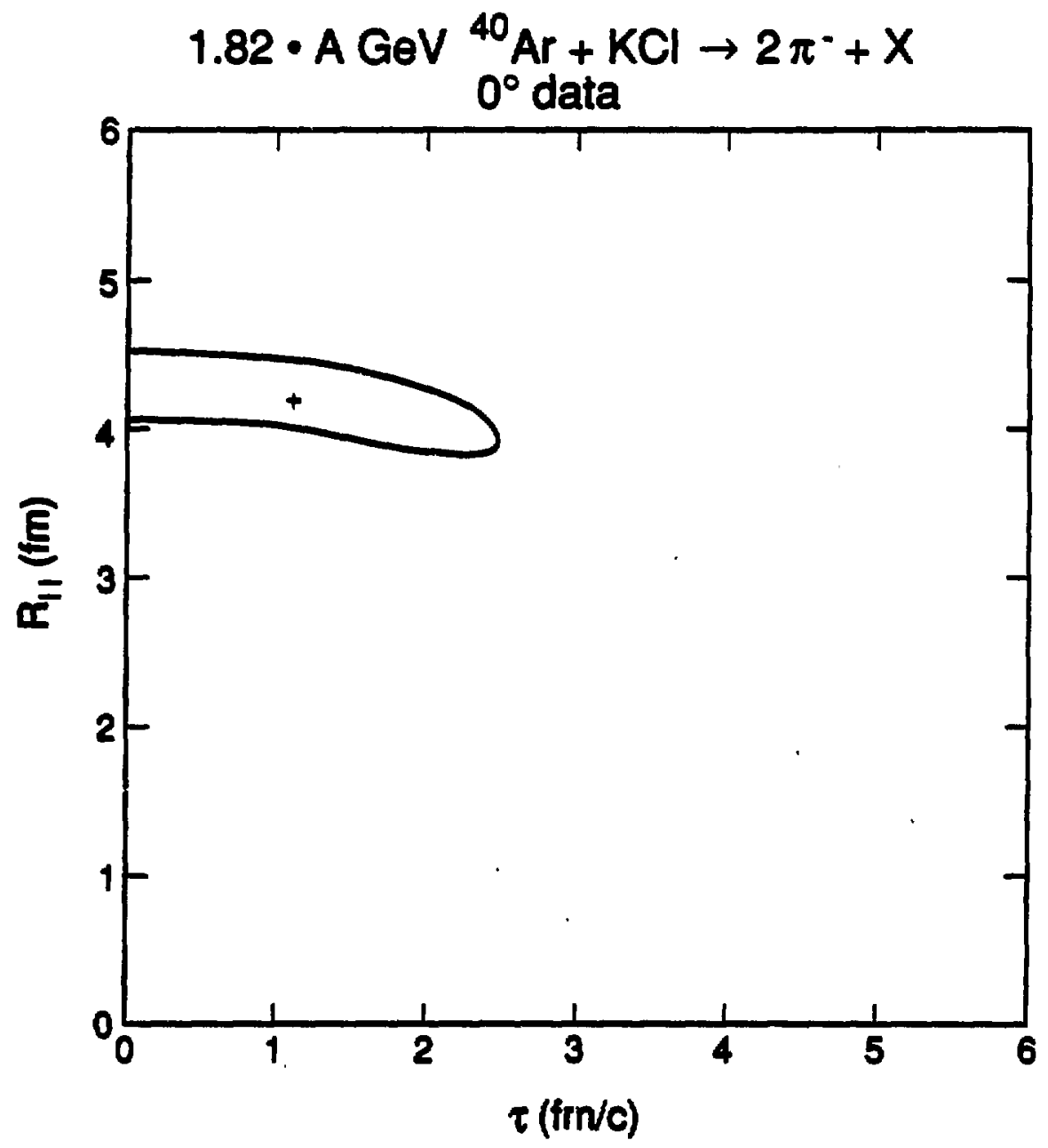

Figure 7.19: The $1 \sigma$ confidence contour for the $0^{\circ}$ Ar least cut date $-R_{\|}$vs. $T$ plane. 


\begin{tabular}{|c|cccc|}
\hline & \multicolumn{4}{|c|}{$\mathrm{Fe}, 0^{\circ}$ data, $\left|\vec{p}_{\text {proj }}\right|>50 \mathrm{MeV} / \mathrm{c}(\sim 10,000$ events $)$} \\
\hline Couiomb correction & In & In & Out & Out \\
\hline Gamow correction & In & Out & In & Out \\
\hline$R_{\perp}(\mathrm{fm})$ & $4.6 \pm 0.2$ & $4.3 \pm 0.3$ & $5.9 \pm 0.5$ & $3.6 \pm 0.6$ \\
$R_{\|}(\mathrm{fm})$ & $1.3_{-1.5}^{+0.7}$ & $0.4 \pm 1.2$ & $2.9_{-0.1}^{+0.3}$ & $2.2_{-1.8}^{+0.8}$ \\
$\tau(\mathrm{fm} / \mathrm{c})$ & $2.8 \pm 0.7$ & $4.2 \pm 0.4$ & $3.7 \pm 1.0$ & $4.2 \pm 1.1$ \\
$\lambda$ & $0.78 \pm 0.04$ & $0.65 \pm 0.06$ & $0.92 \pm 0.08$ & $0.77 \pm 0.07$ \\
$\chi^{2} / \mathrm{NDF}$ & $451 / 421$ & $431 / 421$ & $314 / 286$ & $314 / 285$ \\
$\chi_{\text {fur }}^{2} / \mathrm{NDF}$ & $2046 / 1942$ & $2044 / 1942$ & $1278 / 1247$ & $1280 / 1247$ \\
\hline
\end{tabular}

Table 7.2: Parameters as a function of the corrections applied, for a subet of the $0^{\circ}$ Fe data. Only statiatical uncertalntice are shown.

would allow the corrections to be calculated separately.

To further examine the Coulomb corrections, $\& \pi^{+}$pair dati sample was taken in the $0^{\circ} \mathrm{Nb}$ configuration. In the $\pi^{+}$date sample protons were rejocted if the pulee height in the $A$ and the $B$ counters were both above the cuts for then counters, and the time-of-dight is above the cut for this AB pair. The proton contamination aftor the cuts was estimated by comparing the momentum spectrum without the cuts to the spectrum with the cuts (to determine where the protons were in that spectrum), and comparing the spectrum with cuts to the spactrum of the $\pi^{-}$data analyzed with the same cuts (to determine the spectrum shape in the abeence of the proton contamination). The proton contumination is extimated to be 23\%. The small dats sample size did not allow further reductions in the proton contamination. The agreement between the $\pi^{-}$and the $\pi^{+}$data is at the $1 \sigma$ level for $R_{\perp}, T, \lambda$ and at the $3 \sigma$ level for $R_{\|}$. While this showa acceptable agreement, the uncertainties in the $\pi^{+}$parametern are large, due to the amall data sample size. The parametess (for both the $\pi^{+}$and $\pi^{-}$) are given in Table 7.7.

\subsection{Systematic Uncertainty}

The major source for the systematic uncertainty is believed to be the uncertainty in the residual correlation calculation. The cource of this effect is that the correlation function 
uned as input for the residual correlation calculation is taken from the measurements and is, therefore, uncertain. The systematic uncertainty from this effect was studied for both acceptances. The statistical and syntematic uncertainties for the $0^{\circ} \mathrm{Ar}$ (least cut) and the $0^{\circ} \mathrm{Fo}$ (both cuts) data sets are shown in Table 7.3. Unleas noted, in the remaining tnbles the uncertaintion ahown are the total uncertaintien. Note that this syatematic uncortainty has the foature that Improved atatiatica will decrease the systematic uncertainty by docreasing the uncertainty in the mearured correlation function. The uncertintiea in $\lambda$ due to particle contamination corrections are not included since $\lambda$ was not corrected for the particle contamination.

\begin{tabular}{|c|ccc|}
\hline & Ar, $0^{\circ}$ data & \multicolumn{2}{|c|}{ Fe, $0^{\circ}$ data } \\
\hline & $\left|\tilde{f}_{\text {proj }}\right|>30 \mathrm{MeV} / \mathrm{c}$ & $\left|\vec{p}_{\text {proj }}\right|>30 \mathrm{MsV} / \mathrm{c}$ & $\left|\vec{p}_{\text {proj }}\right|>100 \mathrm{MoV} / \mathrm{c}$ \\
\hline$R_{\perp}(\mathrm{fm})$ & $4.8 \pm 0.3 \pm 0.07$ & $4.8 \pm 0.2 \pm 0.05$ & $4.7 \pm 0.3 \pm 0.08$ \\
$R_{\|}(\mathrm{fm})$ & $4.2 \pm 0.4 \pm 0.2$ & $2.7 \pm 0.3 \pm 0.1$ & $2.1 \pm 0.5 \pm 0.16$ \\
$r(\mathrm{fm} / \mathrm{c})$ & $1.1_{-1.1}^{+1.4} \pm 0.4$ & $2.7 \pm 0.6 \pm 0.2$ & $3.5 \pm 0.6 \pm 0.3$ \\
$\lambda$ & $0.81 \pm 0.05 \pm 0.03$ & $0.88 \pm 0.03 \pm 0.015$ & $0.75 \pm 0.05 \pm 0.02$ \\
$\chi^{2} / \mathrm{NDF}$ & $581 / 537$ & $939 / 729$ & $470 / 395$ \\
$\chi_{\text {ruz }}^{2} / \mathrm{NDF}$ & $2979 / 2590$ & $2938 / 2420$ & $1476 / 1300$ \\
Events & 12900 & 32000 & 11200 \\
\hline
\end{tabular}

Table 7.3: Statistical uncertainties followed by eatimated systematic uncertaintien for the Ar and $\mathrm{Fe} \mathrm{O}^{\circ}$ data sets.

\subsection{The Parameters}

The projections and the confidence coatoura show that the data are well described by the correlation function chosen for the fits, so the remaining fits will not show the projections or the confideace contour plots.

Table 7.4 and Figure 7.20 precent the data for the $45^{\circ}$ set up where $R_{\perp} \equiv R_{\|}$has been forced in the codes 20 that this data is easily compared to earlier data. Note that if $R_{\perp}=R_{\mu}=R$, then

$$
q_{\perp}^{2} R_{\perp}^{2}+q_{\|}^{2} R_{\|}^{2}+\varphi_{0}^{2} r^{2}=\vec{q}^{2} R^{2}+q_{0}^{2} \tau^{2}
$$






Figure 7.20: Farameters as a function of $A$ for the $45^{\circ}$ date with $R_{\perp}=R_{\|}$. The date points for As have been displaced for clarity. Only statistical uncertaintien are shown. 


\begin{tabular}{|c|cccc|}
\hline & \multicolumn{4}{|c|}{$45^{\circ}$ data, single radius parameter fits } \\
\hline Projectile & Ar (Zajc) & Ar & Fe & Nb \\
\hline$R(\mathrm{fm})$ & $2.77_{-0.6}^{+0.6}$ & $1.9 \pm 0.5$ & $2.0 \pm 0.5$ & $3.9 \pm 0.1$ \\
$r(\mathrm{fm} / \mathrm{c})$ & $3.44_{-1.5}^{+1.1}$ & $3.6 \pm 0.8$ & $4.7 \pm 0.5$ & $5.8 \pm 0.4$ \\
$\lambda$ & $0.63 \pm 0.04$ & $0.75 \pm 0.1$ & $0.65 \pm 0.05$ & $0.88 \pm 0.03$ \\
$X^{2} / \mathrm{NDF}$ & $80.3 / 96$ & $145 / 160$ & $389 / 408$ & $846 / 795$ \\
$X_{\text {fun }}^{2} / \mathrm{NDF}$ & $211.2 / 158$ & $1716 / 1643$ & $2195 / 1927$ & $2607 / 2098$ \\
Events & 6700 & 3300 & 8400 & 39100 \\
\hline
\end{tabular}

Table 7.4: Parametere as a function of the projectile, for the $45^{\circ}$ data. The data marked Ar (Zajc) are the At $\pi^{-}$date of W. A. Zaje ef a..[1]; the rumajaing data are this work. Only atatistical uncertainties are shown.

and these fits are equivalent to the single radius parameters fits used earlier[1, 23, 24, 25, 28]. The fits are labeled by the projectile used, where the beam energies and the targets are as listed in Tables 4.1 and 4.2. The column headed Ar (Zajc) is data from Zajc et al.[1]. The fitting method used in [1] is slightly different in that the data were histogrammed a a function of $q=|\bar{\eta}|$ and $s_{0}$, so that the asumption that $R_{\perp}=R_{\|}$could not be tented. For this reason the NDF is quite different from the values in this work. Note that the data frow this work agrees with the data from the the previous work at the $1 \sigma$ level.

Tuble 7.5 and Figure 7.21 preant the data for the $45^{\circ}$ data where $R_{\perp}$ and $R_{\|}$are allowed to vary reparately. These data have a cut imposed on the magnitude of the center of mase momentum requiring both pione to have momenta greater than the lower limit of the acceptance of the spectrometer. This cut was imposed to remove improperly reconstructed tracks. The value of this cut was $90 \mathrm{MeV} / \mathrm{c}$ for the Ar data, $100 \mathrm{MeV} / \mathrm{c}$ for the Fe data and for the $\mathrm{Nb}$ data the cut was ifnored since no data would have been cut. Since this cat remove no real data, thees data are labelled "uncut".

Table 7.6 and Figure 7.22 present the data for the $45^{\circ}$ cot up as above, however the cut on the center of mase momenta was incresed to $\left|\vec{P}_{\mathrm{cal}}\right|>150 \mathrm{MeV} / \mathrm{c}$ to search for effects that depend on the pion momentum. One such effect would be a pion source size depending on the pion momentum, which would be the result of an expanding pion 


\begin{tabular}{|c|ccc|}
\hline & \multicolumn{3}{|c|}{$45^{\circ}$ data, uncut } \\
\hline Projectile & $\mathrm{Ar}$ & $\mathrm{Fe}$ & $\mathrm{Nb}$ \\
\hline$R_{\perp}(\mathrm{fm})$ & $4.5 \pm 1.0$ & $4.0 \pm 0.65$ & $4.8 \pm 0.55$ \\
$R_{\|}(\mathrm{fm})$ & $1.0 \pm 1.0$ & $1.5_{-0.8}^{+0.35}$ & $3.8 \pm 0.2$ \\
$r(\mathrm{fm} / \mathrm{c})$ & $0.0 \pm 0.3$ & $1.7 \pm 1.7$ & $4.8 \pm 1.0$ \\
$\lambda$ & $0.72 \pm 0.10$ & $0.66 \pm 0.06$ & $0.89 \pm 0.035$ \\
$\chi^{2} / \mathrm{NDF}$ & $138 / 156$ & $381 / 403$ & $846 / 795$ \\
$\chi_{\text {FuL }}^{2} / \mathrm{NDF}$ & $1702 / 1662$ & $2194 / 1925$ & $2612 / 2098$ \\
Eveats & 3300 & 8400 & 39100 \\
\hline
\end{tabular}

Table 7.5: Parameters as a function of the projectile, for the $45^{\circ}$ uncut data.

\begin{tabular}{|c|cc|}
\hline & \multicolumn{2}{|c|}{$45^{*}$ data, $\left|\vec{p}_{\mathrm{cal}}\right|>150 \mathrm{MeV} / \mathrm{c}$} \\
\hline Projectile & Fe & $\mathrm{Nb}$ \\
\hline$R_{\perp}(\mathrm{fm})$ & $4.3_{-0.8}^{+0.6}$ & $5.2 \pm 0.55$ \\
$R_{\|}(\mathrm{fm})$ & $1.5_{-1.0}^{+0.6}$ & $4.1 \pm 0.2$ \\
$\tau(\mathrm{fm} / \mathrm{c})$ & $0.1_{-0.1}^{+2.0}$ & $5.0 \pm 1.1$ \\
$\lambda$ & $0.58 \pm 0.07$ & $0.87 \pm 0.035$ \\
$\chi^{2} / \mathrm{NDF}$ & $362 / 345$ & $742 / 719$ \\
$\chi_{\text {ruc }}^{2} / \mathrm{NDF}$ & $2167 / 1897$ & $2477 / 1945$ \\
Evento & 6900 & 34600 \\
\hline
\end{tabular}

Table 7.6: Parameters as a function of the projectile, for the $45^{\circ}$ cut data. There is not enough data in the Ar sample to fit the data with the cut. 
$45^{\circ}$, Uncut data

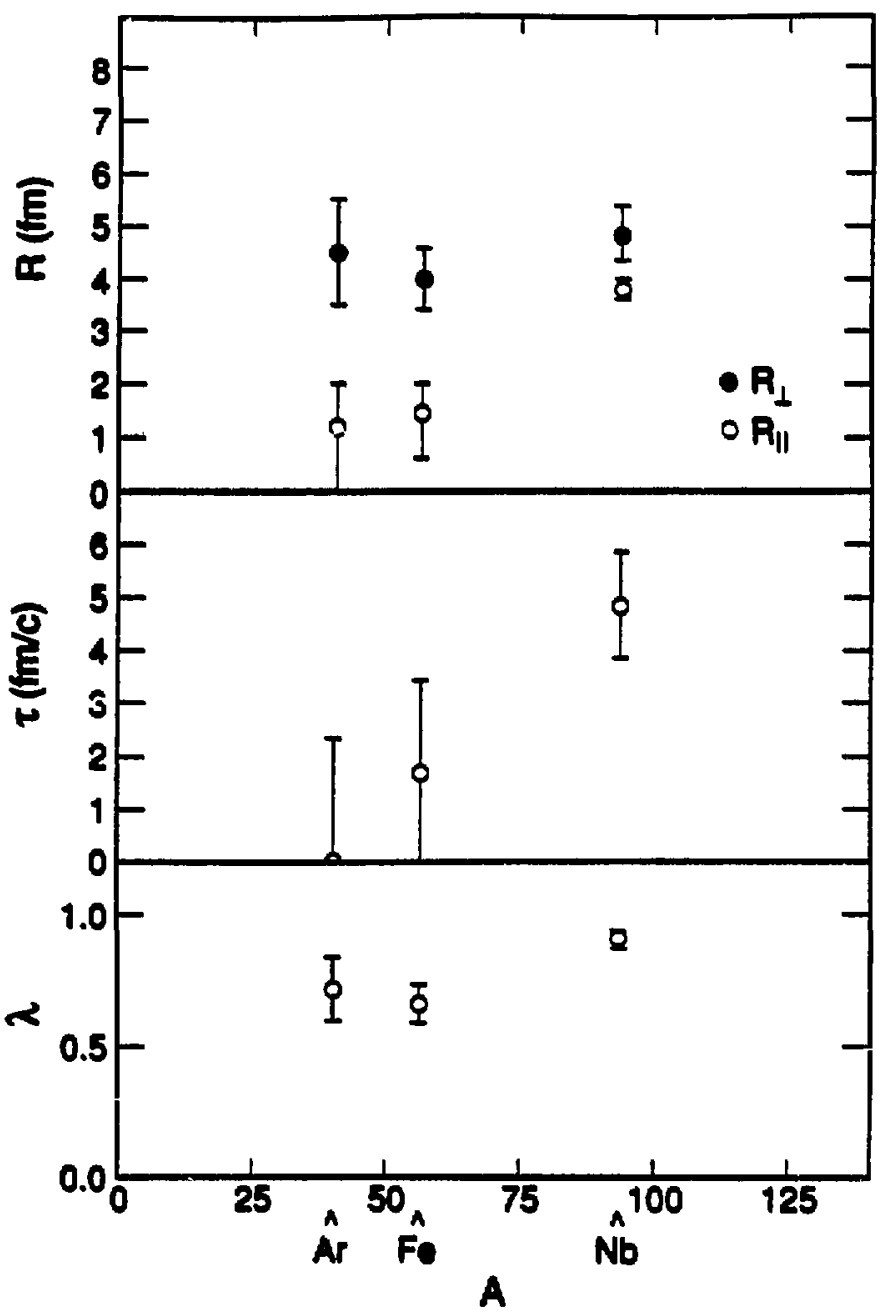

Figure 7.21: Parameters as a function of $A$ for the $45^{\circ}$ uncut data. 


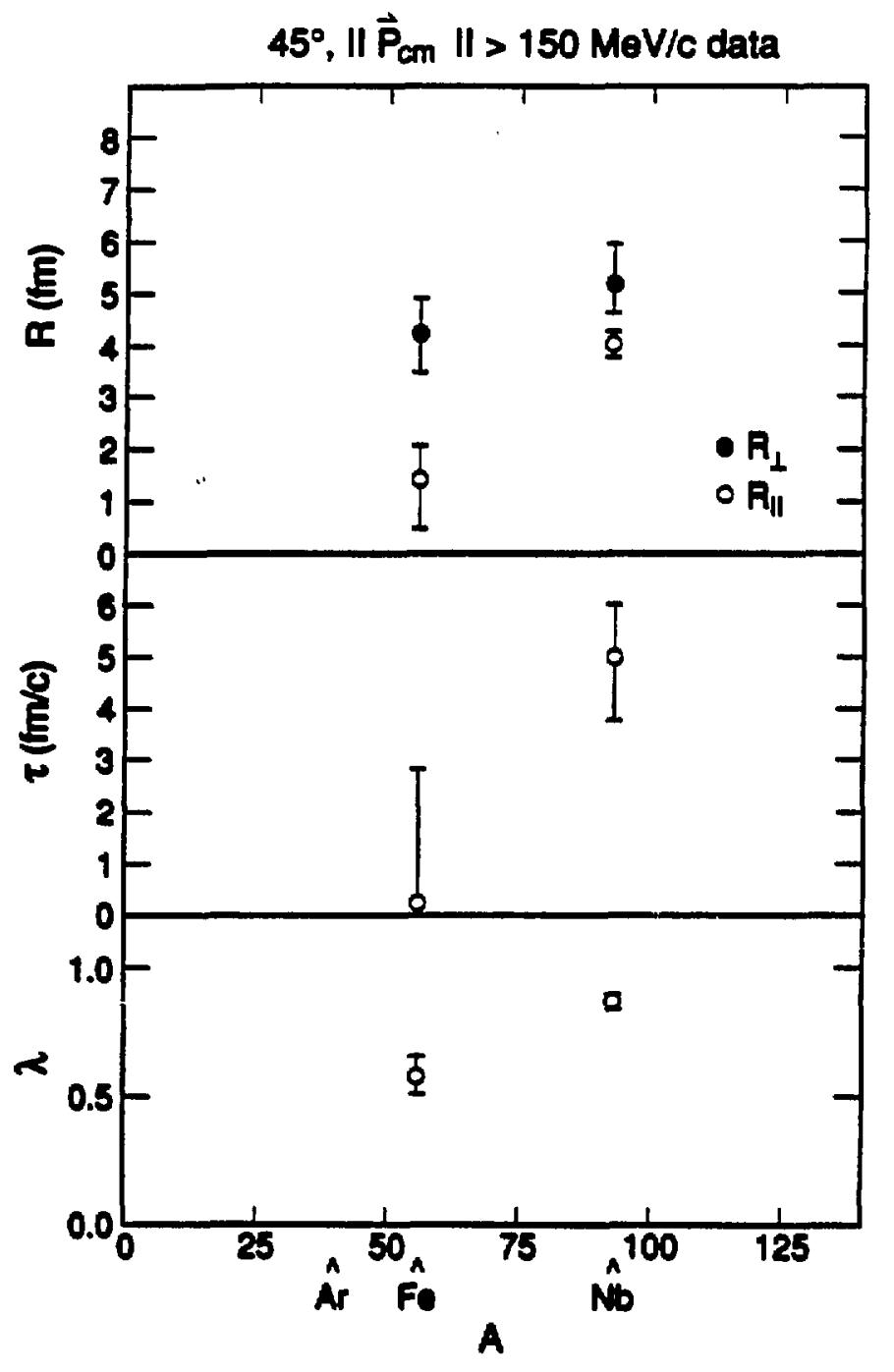

Figure 7.22: Parameters as a funetion of $A$ for the $15^{\circ}$ eut data. 
source (see Section 3.3 and [36]). Examining the corresponding entries in Tables 7.5 and 7.6 shows that, within the uncertainties, there is no dependence on the momentum cut. The Ar data ample was smaller than deaired and there was aot enough data to fit a cut sample.

\begin{tabular}{|c|c|c|c|c|}
\hline & \multicolumn{4}{|c|}{$0^{*}$ data, $\left|\vec{p}_{\text {proj }}\right|>50 \mathrm{MeV} / \mathrm{c}$} \\
\hline Projectile & A: & Fo & $\mathrm{Nb}\left(\pi^{-}\right)$ & $\mathrm{Nb}\left(\pi^{+}\right)$ \\
\hline$R_{\perp}(\mathrm{fm})$ & $4.8 \pm 0.3$ & 2.020 .1 & $5.1 \pm 0.2$ & $5.3 \pm 1.0$ \\
\hline $\boldsymbol{R}_{\mu}(\mathrm{fm})$ & $4.2 \pm 0.4$ & $2.7 \pm 0.3$ & $4.4 \pm 0.3$ & $7.4 \pm 1.2$ \\
\hline$r(f \mathrm{~m} / \mathrm{c})$ & $1.1_{-1.1}^{+1.4}$ & $2.7 \pm 0.6$ & $3.9 \pm 0.4$ & $0.0 \pm-3.0$ \\
\hline$\lambda$ & $0.81 \pm 0.06$ & $0.88 \pm 0.03$ & $1.11 \pm 0.03$ & $1.0 \pm 0.17$ \\
\hline$x^{2} /$ NDF & $581 / 537$ & $939 / 729$ & $1144 / 1087$ & $69 / 86$ \\
\hline$x_{\text {PLL }}^{2} / \mathrm{NDF}$ & $2979 / 2590$ & $2938 / 2420$ & $3776 / 3235$ & $736 / 753$ \\
\hline Events & 12900 & 3200 & 49400 & 1700 \\
\hline
\end{tabular}

Table 7.7: Parameters as a function of the projectile, for the $0^{\circ}$ least cut data.

Table 7.7 and Figure 7.23 present data for the $0^{\circ}$ set up with $\left|\vec{p}_{\text {proj }}\right|>50 \mathrm{MeV} / \mathrm{c}$. The Nb eat up included both $x^{-}$and $x^{+}$data, motivated by a denire to verify the Coulomb coirection calculations as mentioned in Section 7.5 .

Table 7.8 and Figure 7.24 present data for the $0^{\circ}$ eet up with $\left|\vec{p}_{\text {proj }}\right|>100 \mathrm{MeV} / \mathrm{c}$. The larger value for the cut wa motivated by a desire to check for effects that depend on the pion momentum selative to the neareat nuclear matter, the projectile fragment. This would include effects from the Coulomb correction. Note that for the Fe and Nb data the agreement between the two data sets is good. For the As data the higher momentum cut data are about 20 smaller in all parameters.

\subsection{Discussion}

The parameters show the pion source to be oblate $\left(R_{\perp}>R_{\|}\right)$or apherical. Using $24 \pi$ streamer cuamber, Beavis et al. have found spherical sources for $1.5 \cdot \mathrm{A} \mathrm{GeV} \mathrm{Ar}+\mathrm{KCl}$ with parameters[23] of $R_{\perp}=5.0 \pm 0.5 \mathrm{fm}$ and $R_{\|}=5.0 \pm 1.5 \mathrm{fm}$, and for $\mathrm{Ar}+\mathrm{Pb}$ with parametera[25] of $R_{\perp}=3.16 \pm 0.50 \mathrm{fm}, R_{\|}=5.76 \pm 0.54 \mathrm{fm}$ and $\lambda=0.98 \pm 0.14$. The 


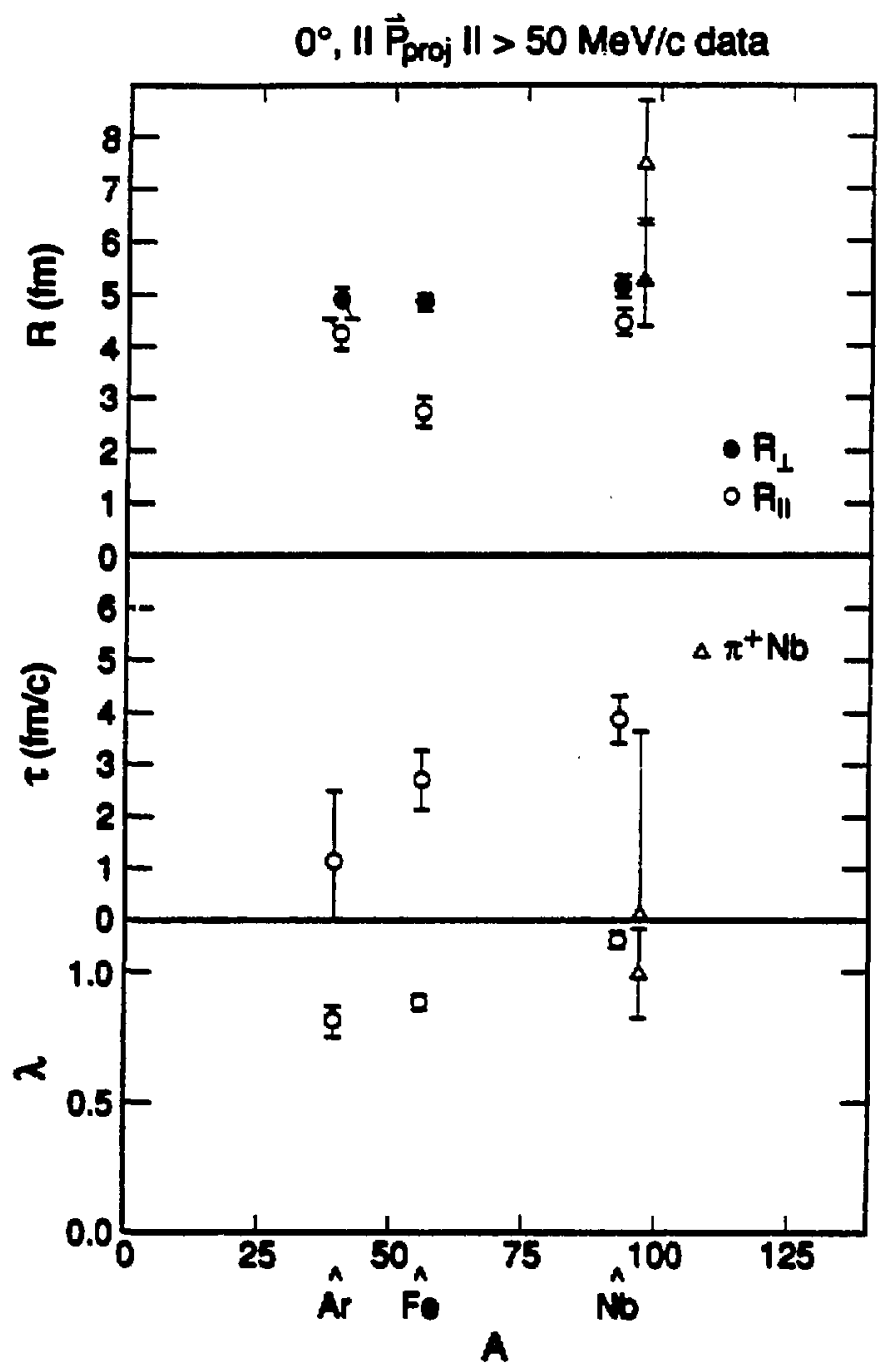

Figure 7.23: Parameters as a function of $A$ for the $0^{\circ}$ last cut data. The data points for Nb have been displaced for clarity. 


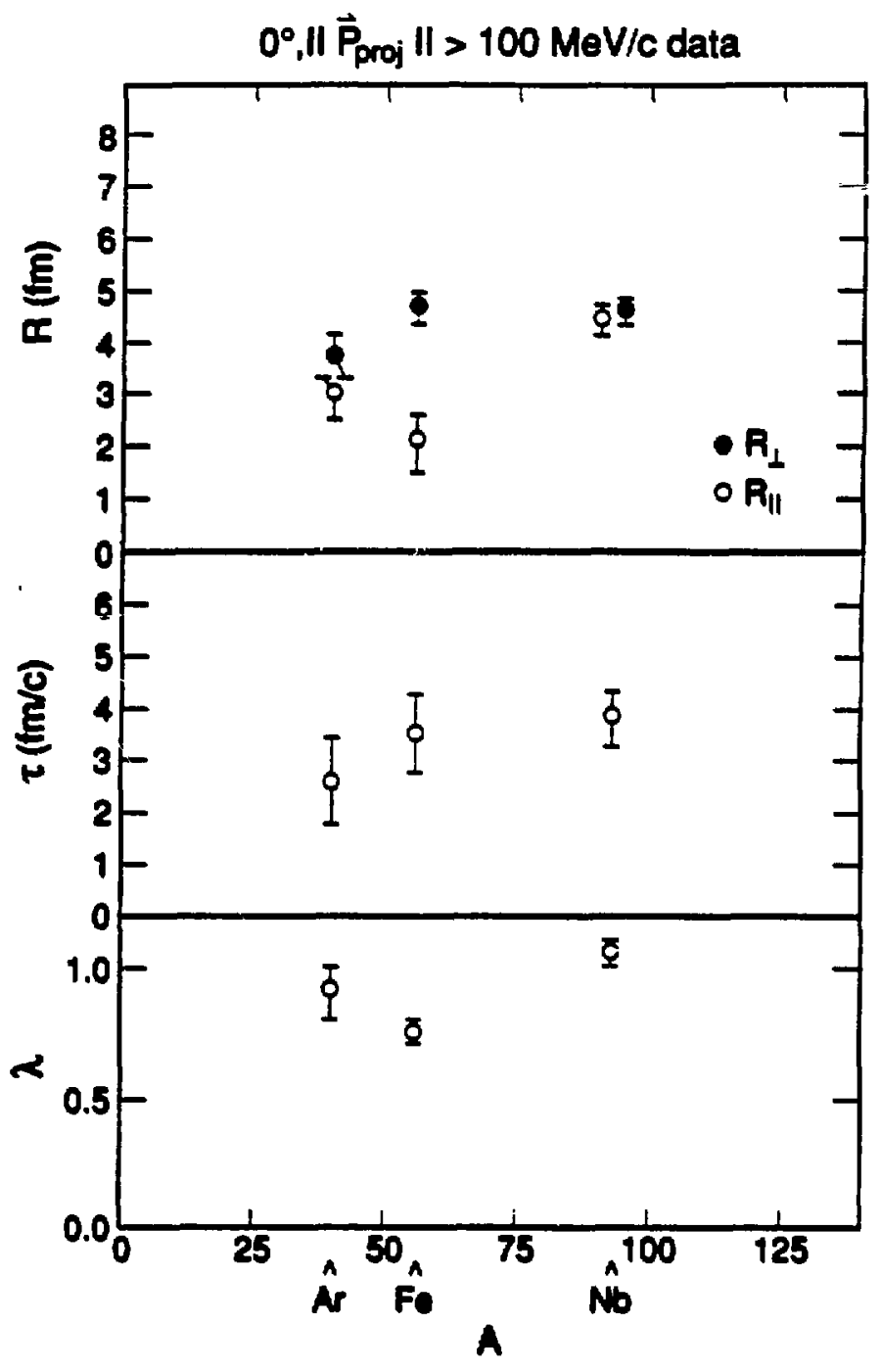

Figure 7.24: Parameters as a function of $A$ for the $0^{\circ}$ cut data. The data pointa for the Nb radii have been displeced for clarity. 


\begin{tabular}{|c|ccc|}
\hline & \multicolumn{3}{|c|}{$0^{\circ}$ data, $\left|\vec{p}_{\text {proj }}\right|>100 \mathrm{MeV} / \mathrm{c}$} \\
\hline Projectile & $\mathrm{Ar}$ & $\mathrm{Fe}$ & $\mathrm{Nb}$ \\
\hline \hline$R_{\perp}(\mathrm{fm})$ & $3.7 \pm 0.4$ & $4.7 \pm 0.3$ & $4.6 \pm 0.3$ \\
$R_{\|}(\mathrm{fm})$ & $3.0 \pm 0.5$ & $2.1 \pm 0.5$ & $4.5 \pm 0.3$ \\
$\tau(\mathrm{fm} / \mathrm{c})$ & $2.7 \pm 0.8$ & $3.5 \pm 0.7$ & $3.8 \pm 0.5$ \\
$\lambda$ & $0.9 \pm 0.10$ & $0.75 \pm 0.05$ & $1.05 \pm 0.04$ \\
$\chi^{2} / \mathrm{NDF}$ & $324 / 318$ & $470 / 395$ & $665 / 630$ \\
$\chi_{\text {PML }}^{2} / \mathrm{NDF}$ & $2147 / 1936$ & $1476 / 1300$ & $2386 / 2091$ \\
Eveats & 6800 & 11200 & 21400 \\
\hline
\end{tabular}

Table 7.8: Parametern a a fuaction of the projectile, for the $0^{\circ}$ cut data.

$\mathrm{Ar}+\mathrm{KCl}$ parameten are within 10 of this thenis, to the oblate shape obtained here is within the uncertaintien of the spherical shape.

At the Interasting Storage Bings (in CERN), Akeason et al.[51] have meaured a prolate source in $\mathrm{p}+\mathrm{p}$ collisions at $\sqrt{5}=63 \mathrm{GeV}$, and spherical cource in He + He collisions at $\sqrt{s}=126 \mathrm{GeV}$. Their data analyais technique is different, becaure they mearure the pion source size perpendicular to the averace of the pions' momenia, and then cut on the direction of the pions' momenta. Their cource sizes (multiplied by $\sqrt{2 / 3}$ to convert to the parametrization und in this thecis) are $R \simeq 1.4 \pm 0.2$ for $p+p$ and $R \simeq 1.2 \pm 0.1$ for He + He.

Uaing the Plastic ball at the Bevalac, Bock et al.[28] found, for $\mathrm{Nb}+\mathrm{Nb}$ at 630. A MeV, $R=3.4 \pm 0.4 \mathrm{fm}$ ( with + fixed at $0 \mathrm{fm} / \mathrm{c}$ and $\chi^{2} / \mathrm{NDF}=1.5$ ) a value 1.25a from the value in this work. However, the single radius parameter $\mathrm{Ar}+\mathrm{KCl}$ fit of Beavis et al. $[23,24]$ gives $R=4.7 \pm 0.5 \mathrm{fm}, r=4.2_{-4.2}^{+1.8}$ and $\lambda=1.2 \pm 0.2$ (the different beam enersy it the reason this fit is not included in Table 7.4). There is aignificant diagreement between these numbers and thove of Zajc et al.[1] and this work.

The parameters for a given acceptance $\left(0^{\circ}\right.$ or $\left.45^{\circ}\right)$ agree, within the uncertainties, for the lower momentum cut and the higher momentum cut. Therefore the data do not show any evidence for possible collective expansion effects[36]. Beavis et al.[25] have also searched for collective expansion effects using cuts on the average of the pions' momenta, but the size of their data sample did not allow any definitive conclusions. Brock et al.[28] 
analyzed their data for variations in the source size as a function of the pions' momenta, but observed only changes in the radius parameter for their $\mathrm{Nb}$ data at the $1 \sigma$ level, again not showing any evidence for expansion.

Comparing the $45^{\circ}$ data and the $0^{\circ}$ least cut $\left(\left|\vec{p}_{\text {proj }}\right|>50 \mathrm{MeV} / \mathrm{c}\right)$ data shows the pion source is more spherical for the $0^{\circ}$ data. The $R_{\perp}$ parameter for one angular acceptance agrees, within the uncertainty, with $R_{\perp}$ for the other acceptance (comparing system by system), 20 this effect is due to $R_{\|}$being contistently amaller in the $45^{\circ}$ acceptance.

Considering the parameters as a function of $A$, for both accoptances, $R_{\perp}$ obows only a weak dopendence on $A$, iacrousing alightly. In fact, within the uncertinties, $R_{\perp}$ could be independent of $A$. The $R_{\|}$parameter dopenda more atroagly on $A$ than $R_{\perp}$. In the 45* acceptance the cource is noticeably oblate for the light syatem, becoming less so for the heavier syatem. In the $0^{\circ}$ acceptance the source shape is slightly oblate, becoming opherical. The $T$ parameter increase with $A$ in the $45^{\circ}$ acceptance (although the uncertaintips are large due to the amall ample sizes), and in the $0^{\circ}$ acceptance this effect is not as clear.

The $\lambda$ parameter is leas than 1 for the $4^{\circ}$ acceptance, for all syatems, with a slight increase with $A$. The $0^{\circ}$ acceptance data show similar behavior, but the values are slightly larger, being juat below 1 for Ar and juat above 1 for Nb.

Compariens of Tables 7.4 and 7.5 show that reatricting the source shape to spherical leaves $\lambda$ unchanged and increanes the extracted $\tau$ by more than 10 . Since the aame data were used in both fits (for a given system), this effect is systematic, not statistical. Future experiments will have to teat the assumption that $R_{\perp}=R_{\|}$, since it is clear that in this experiment the assumption changes the values of parameters other than $R$. 


\section{Chapter 8}

\section{Conclusions and Future Work}

\subsection{Conclusions}

The method of pion-pion interferometry was used to measure the pion source parametera for three nuclear species, uning two acceptances. The methods and computer codes uned in this work have been verified, by Monte Carlo simulations, to give parameters that are within the statistical uncerteinties of the values used a input for the Monte Carlo simulation.

The large data samples present in this experiment have allowed the determination of the source shape (that is, have allowed relaxing the assumption that the source is spherical). In addition, the lifetime of the source was measured and, in the larger data sets, is constrained to be non-zero (this has not always been the case in correlation experiments).

Figure 8.1 showa a plot of data inspired by a review article by Bartke[33]. This plot shows the pion source size purameter $R, a$ a function of the atomic number of the projectile $\left(A_{p}\right)$ to the one-third power. This plot contains data from a range of energien, impact parameters, and anymmetries of the projectile and target (although the projectile wa always lighter than the target). The projectiles, targets, source parameters and the sources of the data are listed in Table 8.1. As in the article, our radius parameters have been multiplied by $\sqrt{3 / 2}$ to give RMS radius parameters. Note that the results of this measurement of the radius parameter are generally smaller than the prior measurements and show some increase with $A_{p}^{1 / 3}$. This plot allows the comparison of some of the data of 


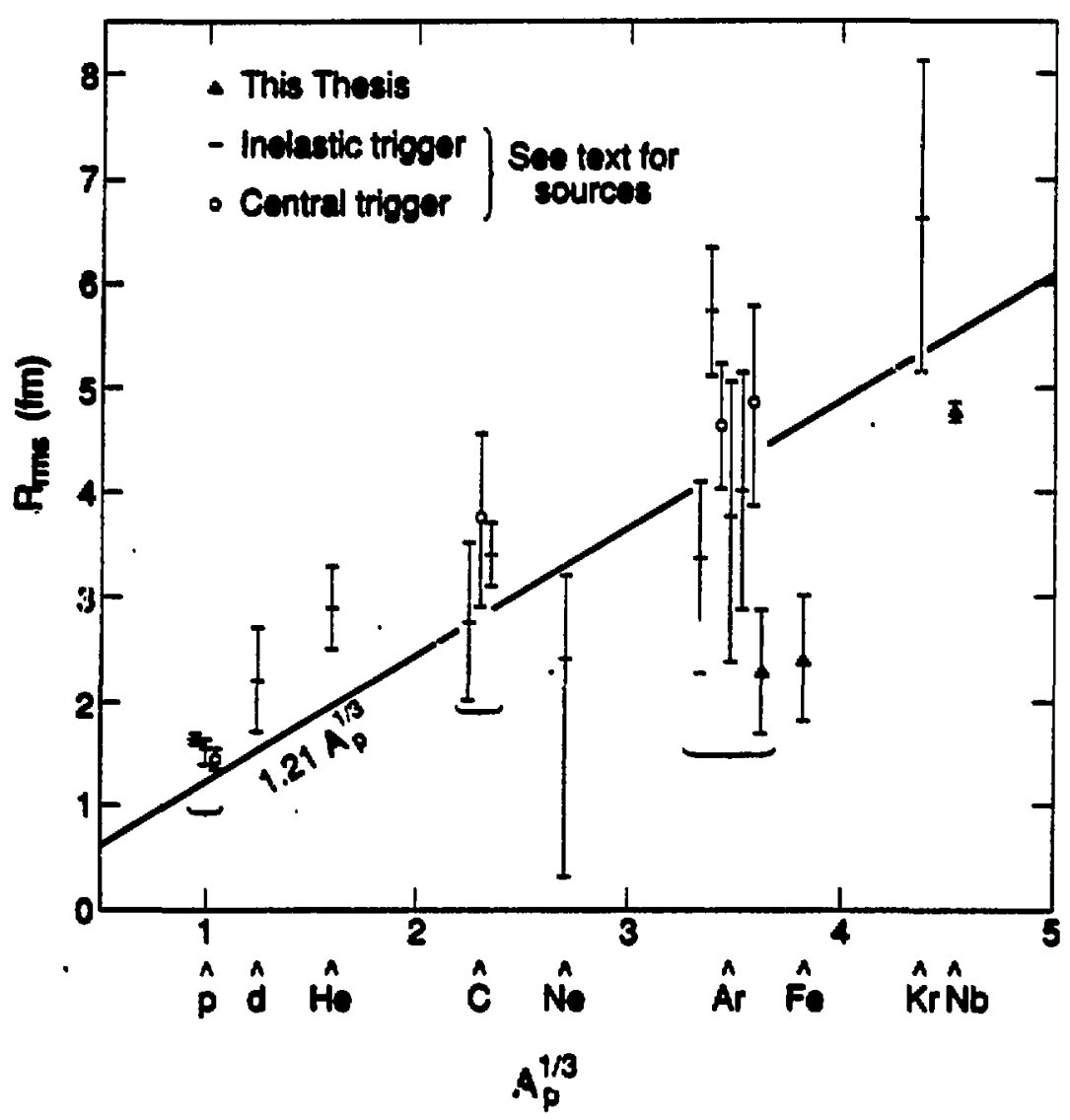

Figure 8.1: Pion source parameters as a fuaction o. $A_{5}^{1 / 9}$, whese $A$, is the atomic number - he projectile. The data are from the cource listed in Table 8.1. Tha data pointa for some projectiles have beer 2 "placed for elarity. 


\begin{tabular}{|c|c|c|c|c|}
\hline$A_{p}$ & $A_{t}$ & $\mathrm{E} / \mathrm{A} \mathrm{GeV}$ & $R_{n=}$ & Reference \\
\hline $\mathbf{p}$ & H & 200 & $1.66 \pm 0.04$ & [54] \\
\hline $\mathbf{p}$ & $\mathbf{x}$ & 200 & $1.53 \pm 0.13$ & [54] \\
\hline * p & $x_{\bullet}$ & 200 & $1.45 \pm 0.11$ & [34] \\
\hline d & $\mathbf{T}$ & 3.4 & $2.20 \pm 0.50$ & [55] \\
\hline He & $\mathbf{T}$ & 3.4 & $2.80 \pm 0.40$ & [55] \\
\hline C & C & 3.4 & $2.75 \pm 0.73$ & [56] \\
\hline - $\mathbf{C}$ & C & 3.4 & $3.76 \pm 0.88$ & [56] \\
\hline C & $\mathbf{T a}$ & 3.4 & $3.40 \pm 0.30$ & [55] \\
\hline $\mathrm{Ne}$ & $\mathrm{NaF}$ & 1.8 & $2.24+1.08$ & [i] \\
\hline $\mathbf{A r}$ & $\mathrm{KCl}$ & 1.8 & $3.399_{-1.10}^{+0.73}$ & [1] \\
\hline Ar & $\mathrm{KCl}$ & 1.5 & $5.76 \pm 0.61$ & [24] \\
\hline * Ar & $\mathrm{KCl}$ & 1.2 & $4.65 \pm 0.61$ & [24] \\
\hline At & $\mathrm{Bal}_{2}$ & 1.8 & $3.75 \pm 1.35$ & [22] \\
\hline Ar & $\mathrm{Pb}_{3} \mathrm{O}_{4}$ & 1.8 & $4.04 \pm 1.14$ & [22] \\
\hline$* A r$ & $\mathrm{~Pb}_{3} \mathrm{O}_{4}$ & 1.8 & $4.87 \pm 0.96$ & [22] \\
\hline At & $\mathbf{k C l}$ & 1.8 & $2.3 \pm 0.6$ & this theais \\
\hline $\mathrm{Fe}$ & Fe & 1.7 & $2.5 \pm 0.6$ & this thetis \\
\hline $\mathbf{K r}$ & $\mathbf{R b B r}$ & 1.2 & $6.61 \pm 1.47$ & [57] \\
\hline $\mathrm{Nb}$ & $\mathrm{Nb}$ & 1.5 & $4.8 \pm 0.1$ & this thesis \\
\hline
\end{tabular}

Table 8.1: Pion source parameters for different projectile target consbinations. The symbole $A_{\text {, and }} A_{t}$ are the projectilo and target atomic aumbern, respectively. The data marked with an anterisk were biand by towards central collisions ubove whatever biasing occurs due to the spectrometer's acceptance. 
this theais to a large sample of pion-pion correlation data. One can see the improvement in the accuracy of the meaurement of the pion source radius parameter for heavy ions in the more recent measurements.

At this point one would, of course, like to point to several theory predictions and pick the one that most ionely agrees with the experimental data. Usually the situation is not so simple and, unfortunately, this experiment is no excoption. The existing theorien are computer Monte Carlo simulations and require too much comput:s time for one person to gererate the required predictions.

There are two nats of data that can be compared wh theory, thowe of the Ar aingle radius parameter fits and the Fe two radius parameter fts. The predictione are taken from the work of Humanic and made using the program CAsCADE. ${ }^{1}$

\begin{tabular}{|c|ccc|}
\hline & \multicolumn{3}{|c|}{ Ar $45^{\circ}$ dath, single radius parameter fits } \\
\hline & Experiment (Zajc) & This Experiment & CASCADE \\
\hline$R(\mathrm{fm})$ & $2.77_{-0.9}^{+0.6}$ & $1.9 \pm 0.5$ & $3.58 \pm 0.11$ \\
$T(\mathrm{fm} / \mathrm{c})$ & $3.44_{-1.5}^{+1.1}$ & $3.6 \pm 0.8$ & $2.83 \pm 0.43$ \\
$\lambda$ & $0.63 \pm 0.04$ & $0.75 \pm 0.1$ & $1.003 \pm 0.045$ \\
$\chi^{2} / \mathrm{NDF}$ & $80.3 / 96$ & $145 / 160$ & - \\
$\chi_{\text {FuL }}^{2} / \mathrm{NDF}$ & $211.3 / 158$ & $1716 / 1663$ & - \\
Events & 6700 & 3300 & - \\
\hline
\end{tabular}

Table 8.2: Comparizon between experimental and CASCADE pion cource parameters for the $45^{\circ}$ data. The data marked (Zajc) are the Ar $\pi^{-}$dats of Zajc et al.[4]; the remaining experimental data are this work. Only statietical uncertainties are shown.

The single radius parameter predictions[27] were generated for comparison to the data of Zajc et al.[1] and the data of Baavis et al.[23, 24]. Table 8.2 presents Humanic'a predictiona, the data of Zajc et al. and the data from tivis experiment (which are taken from Table 7.4). The moasured $R$ is much smaller than the prediction, and $T$ is larger. It is unclear if $\tau$ being larger is a fundamental effect, of an artifact oi fitsing s restricted form of the correlation function. As mentioned in Section 7.8, restricting the form of the fitted correlation function to a single radius parameter causea $r$ to increase. The measured

\footnotetext{
'Ser [27] and refereacen thereila.
} 
values of $\lambda$ are smaller thin the fredicted values.

The Fo data and predictions have ben published earlier[32]. Repeatad here in Table 8.3 are the predictions and the experimental resultn from Tables 7.5, 7.u, 7.7, 7.8. The predicted numbers are generally larger than the sieasured values. This would indicate that a nuclear collision is more than just the superponition of the 2-body interactions, as has been believed for some tiune."

In the abeence of theoretical data, one can try to r.redict what effusts the modifications of the computer codes will have on the piedictions made by the codes. The inclusion of nucleon-nucleon repulsion torms will make the interaction region larger, becaune the aucleona will resiat complession. 'The repulsion will also cause energy to be stored as compresuional energy and be unavalable for pion production. These two effects w. sid lower the energy density available for pion preduction. Asonming that the energy density in the nuclear collision is near the pion production threshold, this would decrese the pion source size, since the energy density in the outer regions of the sollision would drop below the production threshold. So it the nucleir repulvion effect is present in nuclear collisiona, and if the energy density is near the picn production threshold, then the measured source should be smaller and longer lived thas the CASCADE prediction. In Table 8.2 the meanur ad nources are smalier and longer lived than the CAsCADE prediction.

The repultion term will expand the cource perpendicularly to the beam axis, since the increased preasure will puah matter out the sides of the interaction region, giving cources that are more oblate than the CASCADE predictiona. In Table 8.3 it can be seen that the measured sourcee are more oblate than thoee predicted by CASCADE, The nieasured $R_{\perp}$ being equal to or alightly larger than the predichion, while the measured $R_{\|}$is amaller. Here, however, the measured lifetime is shorter than the CAscade prediction.

Collective flow will, to some exteat, cause the momentum distribution of tha pions to peaked in the direction of the flow. This will cause the pion momenta to be correlated in addition to the Bone-Einstein symmetrization, and will cause the measured $\lambda$ to be amaller than the CAsCADE prediction. In Table 8.3 the measured value of $\lambda$ io smaller than the predictions.

Based on these arguments, nucleon-nucleon repulsion effects will be reeded to describe

\footnotetext{
${ }^{2}$ Ses, for exanplt, [52] for an article conceraed specifally with CASCADE.
} 


\begin{tabular}{|c|c|c|c|c|}
\hline & \multicolumn{2}{|c|}{$45^{\circ}$} & \multicolumn{2}{|c|}{$45^{\circ}$} \\
\hline & \multicolumn{2}{|c|}{$\left|\vec{F}_{c}\right|>100 \mathrm{MeV} / \mathrm{c}$} & \multicolumn{2}{|c|}{$\left|\dot{p}_{\mathrm{om}}\right|>150 \mathrm{MeV} / \mathrm{c}$} \\
\hline & Experiment & CASCADE & Experiment & CASCADE \\
\hline$R_{\perp}(\mathrm{fm})$ & $4.0 \pm 0.65$ & $4.2 \pm 0.3$ & $4.3+0.4$ & $4.2 \pm 0.2$ \\
\hline$R_{\|}(\mathrm{Im})$ & $1.5_{-0.6}^{+0.48}$ & $3.0 \pm 0.2$ & $1.5 \pm 0.0$ & $2.9 \pm 0.2$ \\
\hline$r(\mathrm{fm} / \mathrm{c})$ & $1.7 \pm 1.7$ & $3.3 \pm 0.6$ & $0.1 \pm 2.1$ & $3.2 \pm 0.6$ \\
\hline$\lambda$ & $0.66 \pm 0.08$ & $1.00 \pm 0.02$ & $0.38 \pm 0.07$ & $0.98 \pm 0.02$ \\
\hline$x^{2} / \mathrm{NDF}$ & $381 / 403$ & $1099 / 1082$ & $362 / 345$ & $1112 / 1039$ \\
\hline$\chi_{\text {PML }}^{2} /$ NDF & $2194 / 1925$ & $1563 / 1691$ & $2167 / 1897$ & $1596 / 1708$ \\
\hline
\end{tabular}

\begin{tabular}{|c|cc|cc|}
\hline & \multicolumn{2}{|c|}{$0^{\circ}$} & \multicolumn{2}{c|}{$0^{\circ}$} \\
\hline & \multicolumn{2}{|c|}{$\left|\bar{p}_{\text {proj }}\right|>50 \mathrm{MeV} / \mathrm{c}$} & \multicolumn{2}{c|}{$\left|\bar{p}_{\text {proj }}\right|>100 \mathrm{MeV} / \mathrm{c}$} \\
\hline & Experiment & CAsCADr & Experiment & CAsCADE \\
\hline$R_{\perp}(\mathrm{fm})$ & $4.8 \pm 0.2$ & $4.0 \pm 0.1$ & $4.7 \pm 0.3$ & $4.4 \pm 0.1$ \\
$R_{\|}(\mathrm{fm})$ & $2.7 \pm 0.3$ & $3.3 \pm 0.1$ & $2.1 \pm 0.5$ & $2.3 \pm 0.1$ \\
$\tau(\mathrm{fm} / \mathrm{c})$ & $2.7 \pm 0.6$ & $4.3 \pm 0.2$ & $3.5 \pm 0.7$ & $4.4 \pm 0.2$ \\
$\lambda$ & $0.88 \pm 0.03$ & $1.00 \pm 0.02$ & $0.75 \pm 0.05$ & $0.98 \pm 0.02$ \\
$\chi^{2} / \mathrm{NDF}$ & $939 / 729$ & $1031 / 1061$ & $470 / 395$ & $374 / 376$ \\
$\chi_{\text {PLL }}^{2} / \mathrm{NDF}$ & $2938 / 2420$ & $1543 / 1693$ & $1476 / 1300$ & $498 / 555$ \\
\hline
\end{tabular}

Table 8.3: Comparison between experimental and Cascade pion source parameters for the Fe data. The data marked $\left|F_{0}\right|>100 \mathrm{MeV} / \mathrm{c}$ are labeled "uncut" in Chapter 7 , wee Chapter 7. The uncertaintiea shown for the measured data are total. The uncertainties shown for CASCADE are statistical only. 
the pion source size and shape and, therefore, the nuclear collision proress. Theoretical comparions should be made with the results of pion interferometric measurements to help determine if the repulsion terms are being incorporated into the theories correctly.

\subsection{Future Work}

The first question rased by this work is; How does the size of the source incresse with atomic number? The measured source size for $0^{\circ} \mathrm{Ar}$ (with $\left|\vec{p}_{\text {proj }}\right|>50 \mathrm{MeV} / \mathrm{C}$ ) is $R_{\perp}=4.8 \pm 0.3 \mathrm{fm}$ and $R_{\|}=4.2 \pm 0.4 \mathrm{fm}$ and the source size for $\mathrm{p}+\mathrm{p}$ in $\approx 1.4 \mathrm{fm}$ (corrected to the parameterization used in thil thesis) and prolate[51]. Figure 8.1 would seem to indicate that the size increasen emoothly, but at a rate alower than the projectile radius. However, the uncartaintice in some of the measurements are large and there is the additional uncertainty over the anoumption that the source size is dominated by the smaller of the two nuclei in the collision. Measurements (with equal man target and projectile, to make the comparison easier) need to be made in the region of $A \approx 10$ ind $A \approx 20$. Suitable choices for beams and targets are ${ }^{11} \mathrm{~B}+\mathrm{B}$ and ${ }^{20} \mathrm{Ne}+\mathrm{NaF}$.

Given that the $\mathrm{p}+\mathrm{p}$ source is prolate, the Ar source is oblate, and the $\mathrm{Nb}$ source is nearly spherical a econd quention is; How dow the shape of the source depend on the atomic number? For the lower atomic numbers, again, measurements need to be made in the region of $A \approx 10$ and $A \approx 20$, and an experiment using $U+U$ would cover the entire range of particles that the Bevalac accelerator can accelerate (as well as the range of naturally occurring isotopes).

These experix.ents are poasible to do with existing accelerators and detectors, and indeed, could be done with exinting detectors at the Bevalac. Some lighter elements have been measured, but without sufficient statistics to permit a source shape analysis. The Crowe/Rasmuscen Group at LBL has done an experiment using $1.22 \cdot \mathrm{A} \mathrm{GeV}{ }^{130} \mathrm{La}+\mathrm{La}$ (that is under analyais) that may help to answer the question for high atomic numbers, but $\mathrm{U}+\mathrm{U}$ will be needed to cover the hichest atomic numbers ponible.

In the farther future, using $4 \pi$ detectors, it should be poutble to measure all of the charged particles from the nuclear collision and determine the impact plane. One could then measure radius parameters in the impact plane and perpendicular to it. This would remove the necessity for averaging over the impact plane angle that is necessary when 
comparing the current data to the theories. One could also measure these parameters as a function of the impact parameter, since in this type of experiment, the number of participant nucleons will be measured.

As higher energy machines come on line it will become less accurate to ignore the multiparticle conelations since the density of pions in phase-space will be high. The formalism will have to change from the current correlation function, which only considers two pions in the ovent, to perhape comething like speckle interferometry[12]. Theoretical andyois will be needed to determine how to andyes the data to extract the relevant parameters and to dotormine what the relevant parameters are. 


\section{Appendix A}

\section{The Monte Carlo Program}

The performance of the spectrometer was simulated uning Monte Carlo methods. The computer codes used to simulate this experimeat fulfilled three roles. First, the Monte Carlo was used to provide the input data for the ratka fit that gives the track parameters as a function of the wire numbers in the track (eee Section 5.6). Second, it was used to provide fake single track events to teat the hit and track finding software, to measure the acceptance, and to measure the recolution (ane Sections 4.2 and 3.3). Third, it was uned to provide fake two track eveats with a known correlation function to test the correla. tion function fitting procedure and programs (this teat included the entire data analyais atream). These data were also used to check the hit and track finding software when two tracks are present in the event (nee Section 5.4). Many features of the Monte Carlo codes are common to the three roles and the same code, with switchea, was used for all three roles.

The magnetic field map (in. both magnets) was used to track the particles. The fields were interpolated to second order between the grid points and the particles' motion was deacribed by fitting sections from circles for each path atep $(1 \mathrm{~cm}$ in the sir). The separation between the cence planen in the wire chambers was simulated, as was the finite wire-chamber recolution, except for when producing ralka input data when increand accuracy was decired. Multiple scattering and energy lose are simalated using formulas from [58], where the esercy loes can include the Landau distribution (this was switchable). The rimulation did not include misuing wires in the hite, stray particles nor particle contamination. 
For the ERIKA input data, the deaire is to have as a complete representation of the physics as possible without introducing random proceases that would make the output (the wire numbers) a multiple valued function of the input (the momentum and position). For this reason, the random processes were disabled, these are the multiple scattering, and the Landau distribution of the energy lose (energy lose was assumed to happen at the most probable value). The finite wire chamber resolution, while not random, will reduce the accuracy of the zRIKA it, so the finite resolution was not simulated. The accuracy of the fit wa increaced by expending the dimencions of the wire chambars 10\%[1]. The lead walle in the $\mathrm{C}$ magnet wose diabled so that tracks pasing through the lead walls are recoustructed correctly. ${ }^{1}$

For the single track data, the wire chambers are returned to their phyoical size, and the lead walls are re-mabled. The random procenses are re-enabled.

For the two track data, the code is at up a for the single track data, and the momenta of the two pions in the events are correlated according to Equation 3.14. The two track data could optionally be andyzed a twice as many single track events, allowing comparicon of the performance of the software when analyzing one and two track events.

The acceptance is calculated using the procedures for the single track events but, in addition, the pions were weighted by probability of emiasion, using data from [39, 40].

The acceptance is shown in Section 4.2. The one and two track efficienciet are discused in Section 5.4. A fit to Monte Cario correlated data is discussed in Sections 6.9 and 7.2.

\footnotetext{
${ }^{2}$ Swch tracke are remomed from the experimeatel date by requiring the diatence from the track to the surface of the lead wall be pocitive.
} 


\section{Bibliography}

(1] W. Zajc, Phd. theeis, The Univeralty of California, Berkeloy (1982), LBL-14864, and W. Zajc, Phys. Rav. C 20, 2173 (1084).

[2] R. Hanbury-Brown, R. Jeanison and M. Das Gupta, Nature 170, 1061 (1952).

[3] R. Haabury-Brown and R. Twiss, Phil. Mas. (Series 7) 54, 663 (1954).

[4] R. Hanbury-Brown and R. Twise, Nature 177, 27 (1956).

[5] R. Glauber, Phys. Rev. Lett. 10, 84 (1963) and aleo R. Glauber, Phys. Rev. 190, 2529 (1963), R. Glauber, Phys. Rev. 131, 2766 (1963).

[6] J. Armstrong and A. Smith, Phys. Rev, 140 (Number 1A), A155 (1965).

[7] G. Goldhaber, S. Goldhaber, W. Lee and A. Pais, Phys. Rev. 120, 300 (1960). See also G. Goldhaber et al., Phys. Rev. Lett. 3, 181 (1959).

[8] B. Löratad, Int. J. Mod. Phys. A \&, 2861 (1989).

[9] G. Kopylov and M. Podgoretskii, Sov. J. Nucl. Phys. 15, 219 (1972).

[10] E. Shuryak, Phys. Lett. 44B, 387 (1973).

[11] G. Cocconi, Phyı. Lett. 49B, 459 (1974).

[12] W. Zaje, Bace-Einstein Correlations from Statistice to Dynamice, in Hadronic Multiparticle Production, editor P. Carruthers, World Scientific Prea, 1989.

[13] M. Dentechmann et el., Nucl. Phys. B 103, 198 (1976).

[14] M. Deutechmann et al., Nucl. Phya. B 204, 333 (1982). 
[15] M. Adamus et al., Z. Phys. C - Particles and Fields 37, 347 (1988).

[16] G. Goldhaber in Proceedinga on the International Conference on High Energy Physics, Lisbon, Portugal, 1981.

[17] G. Goldhaber in Procendings of the Firat International Conference on Local Equilibrium ea Strong Interaction Phyrics, editors P. Scott and R. Wiener, World Scientiftc Publishing Co., 1985.

[18] G. Goldhaber and I. Jurldic, New Results on the Bose-Einotein Effect in $\mathrm{e}^{+} \mathrm{e}^{-}$Inleractions or the GGLP Effect Revisited, in Procendinge of the Second Intermational Worksbop on Local Equillbrium in Strong Interactions, editors P. Carruthers and D. Strottman, World Scientific Publishing Co., Singapore, 1986.

[19] I. Jurikic, Phd. thenis, The Univernity of Califorain, Berkeley (1987), LBL-24493 and I. Juricić et al., Phys. Rev. D 30, 1 (1989).

[20] B. Andersson and W. Hofmann, Phys. Lett. 160B, 364 (1986).

[21] S. Nagamiya and M. Gyulawy, High Eneryy Nuclear Collisions, in Advances in Nuckar Phyaice (Vol. 3), editore J. Negele and E. Vogt, Plenum Prean, 1984.

[22] S. Fung et al., Phys. Rev. Lett. 41, 1592 (1978).

[23] D. Beavis et al., Phys. Rev. C 27, 910 (1983).

[24] D. Beavis et al., Phys. Rev. C 28, 2561 (1983).

[25] D. Beavis et al., Phys. Rev. C 34, 757 (1986).

[26] J. Ln et al., Phys. Rev. Lett. 46, 898 (1981).

[27] T. Humaxic, Phys. Rev. C 34, 191 (1986).

[28] R. Bock et al., Mod. Phyz. Lett. A 3, 1745 (1988).

[29] W. Christie, Phd. thecis, The University of Californis, Davis (to be published).

[30] A representative, but by no means complete, lint of references is (in chronological ordier): J. Cugnon, T. Mizutani and J. Vandermeulen, Nucl. Phys. A352, 505 (1981); 
E. Braun and Z. Fraenkel, Phys. Rev. C 34, 120 (1986); Y. Kitazoe, M. Sano, H. Toki and S. Nagamiya, Phys. Lett. 166B, 35 (1986); C. Gale, G. Bertach and S. Das Gupta, Phys. Rev. C 35, 1666 (1987); D. Boal and J. Glosli, Phys. Rev. C 37, 91 (1988); J. Molitoris et al., Phys. Rev. C 37, 1014 (1988); and J. Aichelin et al., Phys. Rev. C 37, 2451 (1988).

[31] W. Zajc, Phys. Rev. D 35, 3396 (1987).

[32] A. Chacon et al, Phyı. Rov, Latt. 60, 780 (1988),

[33] M. Gyulasay, S. Kaufimann and L. Wileon, Phys. Rev. C 20, 2267 (1979).

[34] F. Yano and S. Koonin, Phys. Latt. 78B, 356 (1978).

[35] M. Deutschmann et al., CERN/EP/PHYS/78-1 (1978).

[36] S. Pratt, Phys. Rev. Lett. 53, 1210 (1984).

[37] M. Gyulasyy, Phys. Rev. Lett. 48, 454 (1982).

[38] S. Olsen et al., LBL-2445 (1973).

[39] S. Nagamiya et al., Phys. Rev. C 24, 971 (1981).

[40] S. Hayashi et al., Phys. Rev. C 38, 1229 (1988).

[41] J. Harrieon et al., I. E. E. E. Transuctions on Nuclear Science NS-28, 3724 (1981).

[42] A. P. Banford, The Transport of Charced Particle Beams, E. \& F. N. Spon Ltd., London, 1966.

[43] E. Wind, Principle Component Analysio and its Application to Thack Finding, in Formules and Methode in Experimental Data Evaluation (Vol. 3), Editors R. Bock et al., European Physical Society, CERN, Geneva, 1984.

[14] F. James and M. Roos, Nucl. Phys. B172, 475 (1980).

[45] M. Gyulany and S. Kaufimann, Nucl. Phys. As62, 503 (1981).

[46] J. Sulliven private communications, and J. Sulliven et al., Phys. Rev. C 25, 1499 (1982). 
[47] E. Merzbacher, Quantum Mechanics, John Wiley \& Sons, New York, 1970, pp. 245-249.

[48] A. Frodesen, O. Skegsestad and H. Tufte, Probability and Statistics in Particle Physics, Universitetsforlaget, 1979.

[49] F. James and M. Roos, Comp. Phys. Comm. 10, 343 (1975).

[50] F. Jamn, Comp. Phys. Comm. 20, 29 (1980).

[51] T. Akecoon et el, Phys. Lott. B 187, 420 (1987).

[52] J. Molitoris et al., Phys. Rov. C 38, 867 (1986).

[53] J. Bartke, Phyı. Lett. B 174, 32 (1986).

[54] C. DeMarzo et al., Phys. Rev. D 20, 363 (1984).

[35] G. Agakishiev et al., Sov. J. Nucl. Phyı. 30 (3), 344 (1984).

[36] N. Akhababian, J. Bartke, V. Griahin and M. Kowlaski, Z. Phys. C - Particles and Fields 26, 245 (1984).

[57] D. Beavis et al., Multi-Pion Production, in Proc. 7th High Energy Heavy Ion Study (Darmstadt, Oct. 1984), Report GSI-85-10 (Darmatadt, 1985) p. 771.

[58] M. Aguilar-Benitez et al., Particle Properties Data Booklet, North Holland, Amsterdam, 1986. 